\title{
Mitochondrial Aspects of Neuronal Pathology in Triple-Transgenic Alzheimer's Disease Mice
}

\author{
John Zachary Cavendish \\ West Virginia University, jzcav4@gmail.com
}

Follow this and additional works at: https://researchrepository.wvu.edu/etd

Part of the Animal Experimentation and Research Commons, Biochemical Phenomena, Metabolism, and Nutrition Commons, Biological Phenomena, Cell Phenomena, and Immunity Commons, Cell Biology Commons, Laboratory and Basic Science Research Commons, Molecular and Cellular Neuroscience Commons, Nervous System Diseases Commons, and the Neurosciences Commons

\section{Recommended Citation}

Cavendish, John Zachary, "Mitochondrial Aspects of Neuronal Pathology in Triple-Transgenic Alzheimer's Disease Mice" (2021). Graduate Theses, Dissertations, and Problem Reports. 4024.

https://researchrepository.wvu.edu/etd/4024

This Dissertation is protected by copyright and/or related rights. It has been brought to you by the The Research Repository @ WVU with permission from the rights-holder(s). You are free to use this Dissertation in any way that is permitted by the copyright and related rights legislation that applies to your use. For other uses you must obtain permission from the rights-holder(s) directly, unless additional rights are indicated by a Creative Commons license in the record and/ or on the work itself. This Dissertation has been accepted for inclusion in WVU Graduate Theses, Dissertations, and Problem Reports collection by an authorized administrator of The Research Repository @ WVU.

For more information, please contact researchrepository@mail.wvu.edu. 
2021

\section{Mitochondrial Aspects of Neuronal Pathology in Triple-Transgenic Alzheimer's Disease Mice}

John Zachary Cavendish

Follow this and additional works at: https://researchrepository.wvu.edu/etd

Part of the Animal Experimentation and Research Commons, Biochemical Phenomena, Metabolism, and Nutrition Commons, Biological Phenomena, Cell Phenomena, and Immunity Commons, Cell Biology Commons, Laboratory and Basic Science Research Commons, Molecular and Cellular Neuroscience Commons, Nervous System Diseases Commons, and the Neurosciences Commons 


\title{
Mitochondrial Aspects of Neuronal Pathology in Triple-Transgenic Alzheimer's Disease Mice
}

\section{John Z. Cavendish}

Dissertation submitted to the West Virginia University Health Science Center Neuroscience Program in partial fulfillment of the requirements for the degree of

Doctor of Philosophy in Neuroscience

\author{
James W. Simpkins, Ph.D., Chair \\ Werner Geldenhuys, Ph.D. \\ Paul Lockman, Ph.D. \\ Bernard Schreurs, Ph.D. \\ Eric Tucker, Ph.D.
}
Department of Neuroscience, West Virginia University
Morgantown, West Virginia
2019

Keywords: Alzheimer's Disease, Neurodegeneration, 3xTg-AD Mice, Mitochondrial Structure, Mitochondrial Movement, Sigma-1 Receptors

Copyright 2019 John Z. Cavendish 


\title{
Abstract \\ Mitochondrial Aspects of Neuronal Pathology in Triple-Transgenic Alzheimer's Disease Mice \\ John Z. Cavendish
}

\begin{abstract}
Alzheimer's disease (AD) is a fatal, progressive neurodegenerative disease afflicting millions of people in the United States alone and is the only one of the top leading causes of morbidity and mortality with no effective disease-modifying therapies. It is the most common form of dementia, affecting one in three people over the age of 85 . While the hallmarks of the disease include accumulation of beta-amyloid-based extracellular plaques and hyperphosphorylated tau-based intracellular neurofibrillary tangles, treatment strategies centered on removing or mitigating these components of $\mathrm{AD}$ have all failed in humans. Mitochondrial dysfunction has been increasingly recognized as an early and consistent pathological feature of AD. Many questions remain, however, regarding the onset of mitochondrial dysfunction, its importance to disease progression, and its amenability to treatment to slow or halt progression of AD. Using a triple-transgenic mouse model of AD, we studied mitochondrial abnormalities in neurons during the earliest and latest stages of $\mathrm{AD}$ pathology. The studies presented in this dissertation show that mitochondrial dysfunction possibly begins even during neural development and could be amenable to correction with activation of the sigma-1 receptor, an intracellular chaperone molecule that enhances mitochondrial function among other neuroprotective effects. We also show that neuronal mitochondrial number and structure are largely preserved across multiple brain areas at the very latest stages of AD. The studies presented herein add to the growing body of evidence of mitochondrial involvement in AD pathophysiology and suggest possible avenues for clinical translation of mitochondrially-targeted therapies for $\mathrm{AD}$.
\end{abstract}




\section{Dedication}

This dissertation is dedicated to the memory of my father-in-law, Dr. Sheikh Nazir Ahmed, who was one of my greatest role-models and teachers. Although he is no longer with us, his legacy carries on through the countless people he impacted. Dr. Ahmed's unmatched generosity and dedication to his family, community, and profession as an organic chemistry teacher continue to inspire all who knew him. راجعون إلـ يه إنّاوَ للهِ إنّاّ (We belong to God and to Him we shall return).

I also dedicate this dissertation to my wife, Nadia Ahmed. Throughout most of my adult life, Nadia has been there for me, providing love, support, motivation, and criticism when I needed it. We have grown together through the difficulties and good times of life, and I could not ask for a better partner as we go forward on our journey.

Finally, this dissertation is dedicated to my parents, John and Niki, who worked tirelessly to give me a good life and helped me become the person I am today, and to my children, Arif and Ahmed, who have been my greatest joys and have given me a new appreciation for what my parents did for me throughout my life. 


\section{Acknowledgements}

I would like to first thank my mentor and advisor, Dr. James Simpkins, for his unwavering support and belief in me, his insight and wisdom, and his guidance throughout my training. Dr. Simpkins has devoted immeasurable time and energy to help me overcome the obstacles I encountered in graduate school and learn to become a successful researcher.

I would also like to thank current and former members of the Simpkins lab, including Sophie Ren, Elizabeth Engler-Chiurazzi, Heng Hu, Stephanie Rellick, Sujung Jun, Deborah Corbin, Sara Lewis, Dominic Quintana, Ashley Russell, Keyana Porter, Mark "Alex" Colantonio, and Brishti White. I would especially like to thank Dr. Saumyendra Sarkar who was a close mentor and scientific advisor, and whose insight and wisdom was integral to my training.

I would like to thank my current and past committee members, Dr. Eric Tucker, Dr. Paul Lockman, Dr. Bernard Schreurs, Dr. Werner Geldenhuys, Dr. George Spirou, and Dr. Charles Rosen, for their constant guidance and support. I would also like to thank my former advisor Dr. Aric Agmon for providing me with my initial training and continuous advice throughout graduate school.

I thank Dr. Albert Berrebi, Dr. Lisa Salati, Dr. David Siderovski, and Dr. Fred Minnear for their direction of the graduate and M.D./Ph.D. programs and their dedication to helping students succeed.

I sincerely thank my mother-in-law, Tamanna Ahmed, for supporting me throughout my Ph.D. and helping me manage life away from the lab. Her constant help, pleasant company, and loving care for our children has helped us get through the toughest of times. We are forever grateful to have her in our lives.

Finally I thank my family and friends for always being there for me to provide love, help, and support when I need it the most. 


\section{Table of Contents}

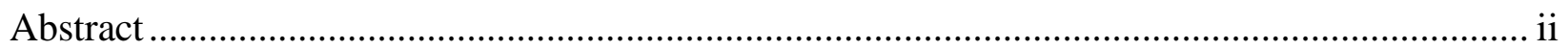

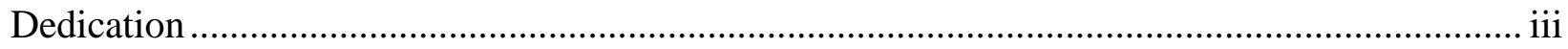

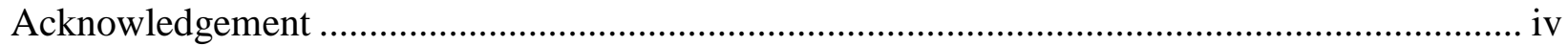

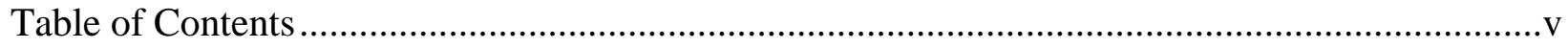

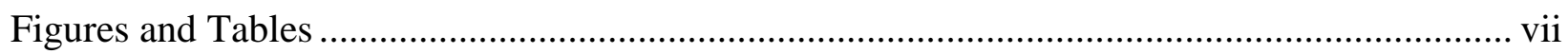

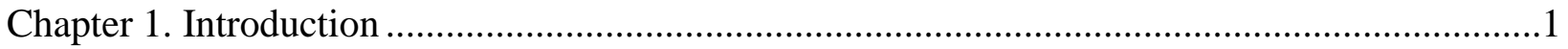

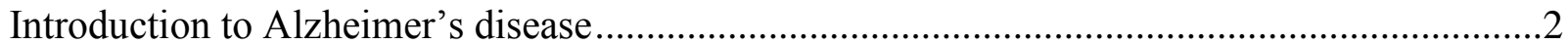

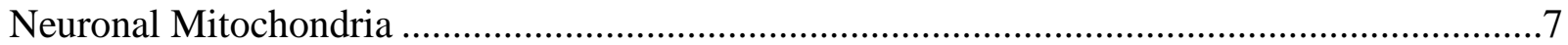

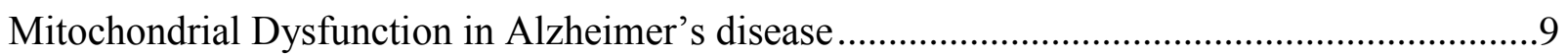

Sigma-1 Receptors for Treatment of Alzheimer's disease .....................................................12

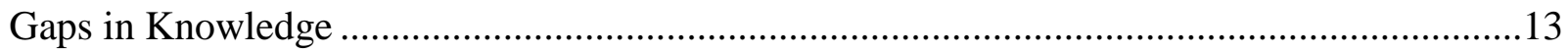

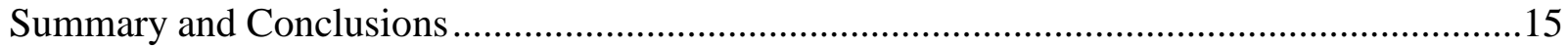

Chapter 2. Mitochondrial Movement and Number Deficits in Embryonic Cortical Neurons from

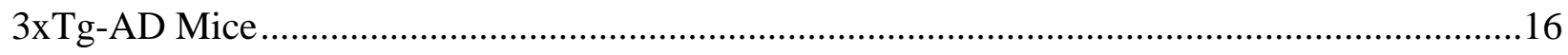

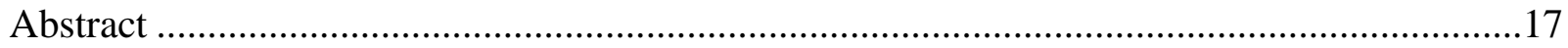

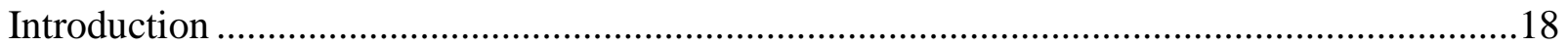

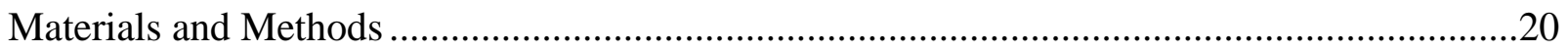

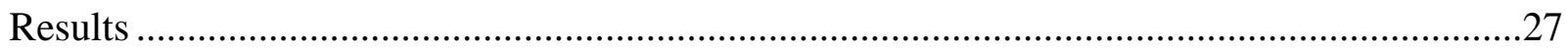

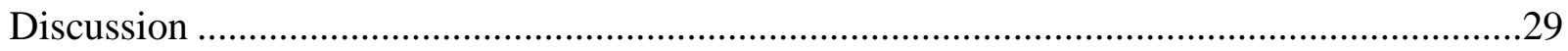

Chapter 3. Mitochondrial Structure and Number in Cortical and Hippocampal Neurons of Aged

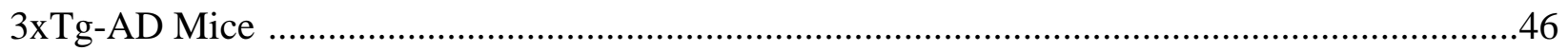

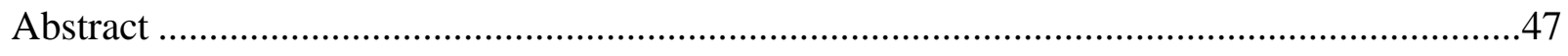

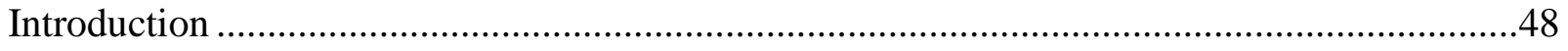

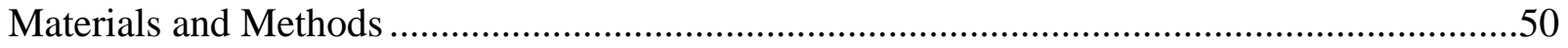

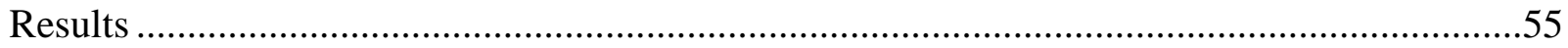

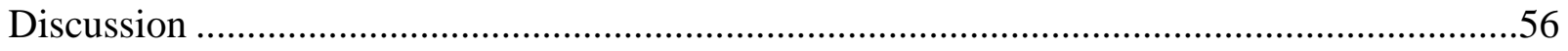




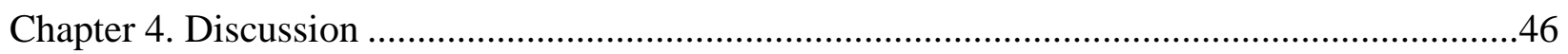

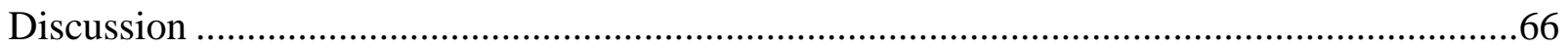

Mitochondrial Changes Early in Development of Alzheimer's Disease ..............................67

Sigma-1 Receptor Agonists for Treatment of Alzheimer's Disease ...................................69

Preservation of Mitochondrial Structure and Number Late in Alzheimer's Disease ................71

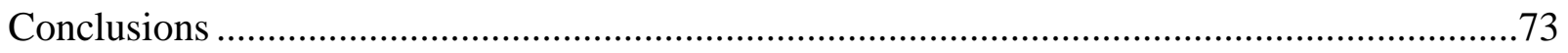

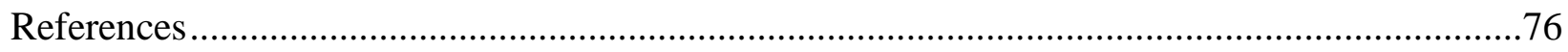




\section{Figures}

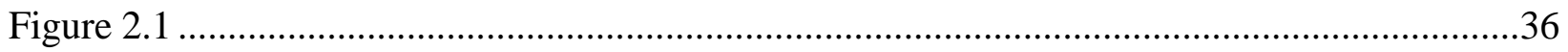

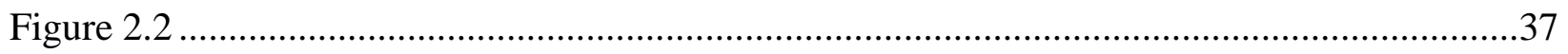

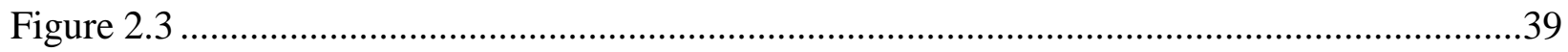

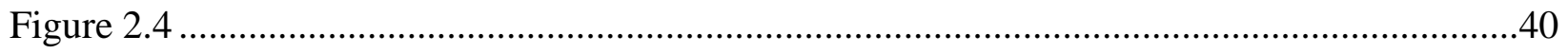

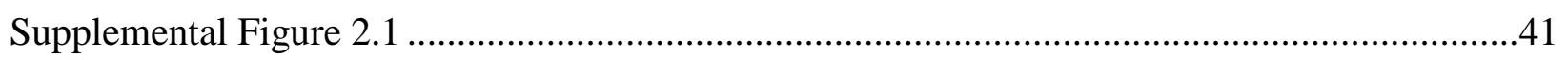

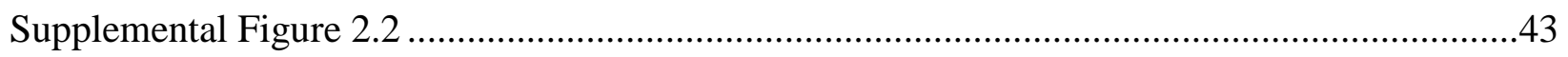

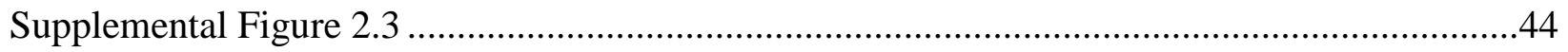

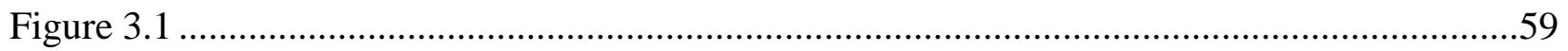

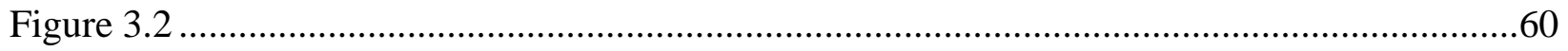

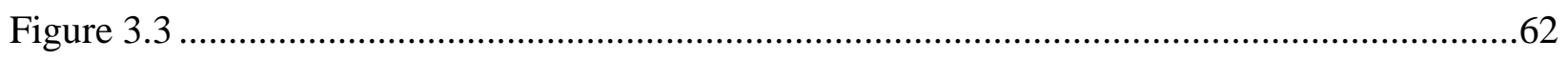

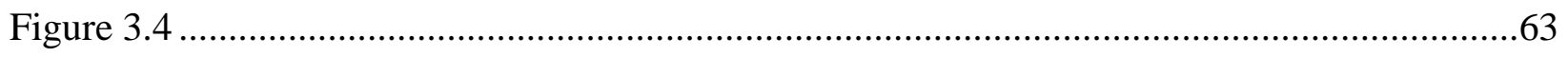

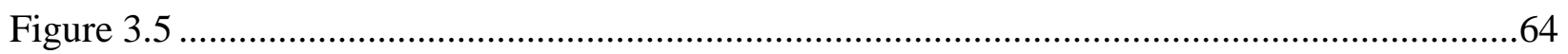

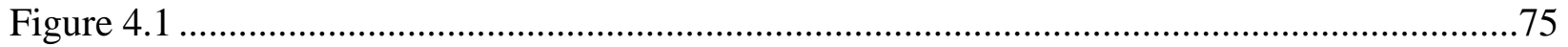




\section{Chapter 1}

\section{Introduction}




\section{Introduction to Alzheimer's disease}

\section{Epidemiology and Impact}

Alzheimer's disease (AD) is the leading cause of dementia and the sixth-leading cause of death in the United States, but it should be noted that the number of deaths caused by AD are likely underestimated due to reporting of other illnesses which stem from AD as the primary cause [1]. The number of people currently afflicted with AD in the United States alone is approaching 6 million, and ten percent of people 65 and older have AD [1]. At age 85, one in three people will have the disease [1]. With the increase in elderly individuals from the baby boom generation, these numbers are expected to increase dramatically. By 2050, it is projected that up to 14 million US adults will have AD if there are no new breakthroughs in disease prevention or treatment [1]. Roughly two-thirds of AD patients are female, but the incidence of developing AD appears to be roughly the same between men and women. Women, however, tend to live longer and have less chance of cardiovascular mortality in middle-age, so a survivor bias could be the reason for the discrepancy in AD prevalence between the sexes [1].

It has been over a century since the disease was first described, yet AD remains the only one of the top ten causes of death with no cure and no treatment which slows disease progression. It is also the only one of the leading causes of death that is consistently increasing in its mortality rate, with at least a 145 percent increase since the year 2000 [1]. Because of the progressive nature, high degree of disability and dependence, and often long duration of the disease, AD carries a large burden on patients, caregivers, and the healthcare system. The cost of $\mathrm{AD}$ in monetary terms is currently about a quarter of a trillion dollars per year, and could rise to a trillion dollars per year by 2050, not including an equally high cost of unpaid caregivers who are often friends, family members, and volunteers [1]. The real burden of the disease, however, is immeasurable. Throughout the course of $\mathrm{AD}$, patients lose the ability to remember past experiences, recognize loved ones, carry out daily activities at home and in society, plan for the future, and emotionally respond to the joys and challenges of life. This is hard for not only the patients, 
but loved ones and caregivers who have a first-hand view of the progressive, yet often unpredictable deterioration of the mind and body of someone afflicted with AD.

\section{Progression of $A D$}

The clinical symptoms of AD begin with very subtle cognitive changes, sometimes not even measurable by traditional cognitive screening methods. Patient-reported feelings of cognitive impairment, known as subjective cognitive decline, are now recognized as a risk factor for development of dementia in later life [2]. Biomarkers such as $A \beta_{42}$, total tau, and phosphorylated tau may also be present in cerebrospinal fluid well before the onset of clinical symptoms and also represent an increased risk of developing AD [2]. Positron emission tomography (PET) scanning can also detect potential pathological changes in the very early stages of AD. The two main PET findings are reduced cerebral glucose metabolism [3] and presence of binding to $\mathrm{A} \beta$ of the Pittsburgh compound $\mathrm{B}(\mathrm{PiB})$ [4]. The next stages of $\mathrm{AD}$ consist of progressive cognitive impairment that can be detected by clinical cognitive screening methods. Mild cognitive impairment (MCI) is the first clinically detectable stage, and patients with MCI are at high risk for developing dementia later in life [2]. MCI manifests differently for different people, but can include difficulty forming new memories, loss of short-term memory, speech impairment, difficulty following complex instructions, misplacing items, and a range of effects on ability to carry out job-related or routine daily activities. As MCI progresses to dementia, patients experience more severe and profound memory loss, including the inability to recognize people, things, or places that were once intimately familiar to them. Impairment in the ability to perform activities of daily living lead to dependence on caregivers or admission to long-term care facilities. Emotional symptoms such as aggression, frustration, and depression also often occur. In the late stages of disease, AD patients become increasingly immobile and dependent on care, and often die of secondary causes due to this such as infections or cardiovascular complications. There is ultimately a 100 percent mortality rate from AD. Postmortem analysis of brain pathology can differentiate AD from other dementias, but it is noteworthy 
that some people can show all of the brain pathology of AD with no clinical symptoms [5, 6]. Conversely, some patients with clinical signs of AD do not present significant AD brain pathology [7].

\section{Pathophysiology of $A D$}

Since the 1980s, the National Institutes of Health, primarily through the National Institute of Aging, have been devoting ever-increasing amounts of funding to AD research. Although important strides have been made in understanding more about the etiology of $\mathrm{AD}$, the precise mechanisms of $\mathrm{AD}$ pathophysiology are poorly understood, and only a handful of pharmaceutical treatments have been approved which only modify disease symptoms but do not slow or stop disease progression. When AD was first described in 1906 by the German physician, Alois Alzheimer, from whom AD gets its name, two pathological hallmarks were described: extracellular "plaques" and intracellular "tangles" [8]. It is now known that the plaques of AD stem from aggregation of the beta amyloid (A $\beta$ ) peptide which is cleaved from the membrane-bound amyloid precursor protein (APP) by beta- and gamma-secretases [2]. Extracellular amyloid plaques are perhaps less toxic than the intracellular oligomers of $A \beta$ that form earlier in disease progression. The neurofibrillary tangles of $\mathrm{AD}$ are initiated by and consist primarily of aggregates of the microtubule-associated protein tau, which is provoked by hyper-phosphorylation at multiple residues on the protein [9]. Other well-known pathological changes of AD include atrophy of the cerebral cortex and hippocampus with enlargement of ventricles, loss of synapses, and disruption of neuromodulatory systems, specifically the cholinergic nucleus basalis of Meynert $[10,11]$. More recently, other pathophysiological changes have been identified in $\mathrm{AD}$, including vascular pathology, increased inflammation, and metabolic deficiencies related to mitochondria [12-14].

\section{Risk Factors for $A D$}

There are two types of AD — familial, early-onset AD, and sporadic, late-onset AD. Familial AD is caused by mutations in the genes PSEN1/PSEN2, encoding the presenilin 1 and 2 proteins (PS1 and PS2) which make up subunits of the gamma secretase complex, and APP, from which A $\beta$ is derived [2]. 
Familial AD is dominantly inherited and leads to disease in most cases by the age of 45 [2]. The causes of sporadic $\mathrm{AD}$ are not known, but some genetic and environmental risk factors have been identified. The biggest risk factor is age, but others include presence of APOE $\varepsilon 4$ allele, smoking and lifestyle factors, diabetes, poor cardiovascular health, and stroke. Some of the hallmarks of Alzheimer's disease are also seen in chronic traumatic encephalopathy [15], which further suggests that brain trauma may be a risk factor for AD. Sporadic AD becomes more common at the age of 65 and increases continuously with age.

\section{Amyloid Cascade Hypothesis and Amyloid-based Treatments}

The amyloid cascade hypothesis has been a prominent theory of both sporadic and familial AD pathophysiology for over 25 years $[16,17]$. This hypothesis proposes that increased production and/or reduced clearance of $A \beta$, specifically $A \beta_{42}$ leads to oligomerization of $A \beta$ intracellularly. Intracellular $A \beta$ oligomers bind to a wide variety of targets in neurons leading to activation of multiple pathways detrimental to neuronal function and survival. These include impairment of synaptic proteins, increased reactive oxygen species production, endoplasmic reticulum stress, mitochondrial dysfunction, and apoptosis. Amyloid oligomers are also thought to bind and activate kinases that phosphorylate tau and lead to neurofibrillary tangles. Extracellular amyloid plaques which occur in later stages are also thought to activate the inflammatory response of microglia which further damage synapses and neurons.

There have been several clinical trials of treatments aiming to reduce production or increase clearance of $\mathrm{A} \beta$ to improve $\mathrm{AD}$ symptoms and halt disease progression. To date, not a single trial has produced successful results, and some have had to be stopped early due to worsening outcomes in treated groups.

\section{In Vitro and Animal Models of $A D$}

Studying the pathophysiology of $\mathrm{AD}$ and potential treatments in humans is limited by the fact that $\mathrm{AD}$ likely begins to develop decades before the onset of symptoms, perhaps even during very early brain development in some cases. Many of the studies needed to document early changes the precede MCI and 
$\mathrm{AD}$ would be unethical or impossible in healthy adults. Models allow these barriers to be overcome, but have their own limitations as well.

In vitro models of $\mathrm{AD}$ are useful for studying detailed cellular and molecular biological aspects of the disease as well as providing an opportunity for high-throughput screening of potential therapeutic compounds. Applying exogenous A $\beta$ species such as oligomers to cultured neuron-like cell lines or primary neurons allows studying toxic effects of amyloid [18-20]. Overexpression of mutant tau species, such as tau $301 \mathrm{~L}$, prone to hyperphosphorylation can provide a tool to study the mechanisms and intracellular effects of neurofibrillary tangle formation in live cells and probe potential treatments [21]. In vitro models, however, do not allow exploration of the cognitive symptoms of $\mathrm{AD}$ or the effects of treatments on the level of the whole brain and/or organism. The delivery of drugs in in vitro models is not limited by the pharmacokinetics of whole organisms and do not need to pass through the blood-brain barrier, so success of compounds in vitro does not always predict that they will be useful for treatment of $\mathrm{AD}$ in vivo.

Animal models can partially overcome the challenges of in vitro models. Most animal models of $\mathrm{AD}$ have been created in mice [22]. Because the etiology of sporadic AD is largely unknown, researchers used genes mutated in the familial form of $\mathrm{AD}$ to recapitulate aspects of $\mathrm{AD}$ in transgenic mice. These mice are therefore not a true model of the more common sporadic $\mathrm{AD}$, but do help the understanding of how $\mathrm{A} \beta$ and/or tau pathology, associated with both sporadic and familial $\mathrm{AD}$, cause downstream effects. They also offer a vehicle for testing potential treatments at very early stages of $\mathrm{AD}$, before overt symptoms or neuropathology are present. The first transgenic mouse models used mutant APP or a combination of mutant APP with PS1, and these mice showed an age dependent development of extracellular plaques and cognitive deficits similar to human patients. To model neurofibrillary tangles as well as plaques, additional transgenes were added. One of the most commonly used AD mouse models is a triple-transgenic $\mathrm{AD}(3 \mathrm{xTg}-\mathrm{AD})$ mouse that was developed by injecting the tau $\mathrm{P}_{301 \mathrm{~L}}$ and $\mathrm{APP}_{\mathrm{Swe}}$ mutant genes under the Thy1.2 mouse promoter into single-cell embryos from PS1 $1_{\mathrm{M} 146 \mathrm{~V}}$ homozygous knock-in 
mice [23]. This mouse recapitulates both the plaques and tangles of $\mathrm{AD}$ as well as cognitive deficits with aging. The A $\beta$ first accumulates intracellularly, which is associated with synaptic physiological changes, and then continues to accumulate extracellularly. Finally, intracellular hyperphosphorylated tau pathology is seen, starting in the hippocampus and progressing to the cortex [24].

Transgenic mice have some disadvantages. One problem is that the insertion of transgenes may disrupt genes at the insertion point in the genome, which could lead to confounding effects not caused directly by amyloid or tau pathology. Another problem is that no transgenic model to date has been able to fully recapitulate every single aspect of human disease. 3xTg-AD mice, for instance, do not show any increased mortality compared to non-transgenic controls. Nevertheless, mouse models such as 3xTg-AD mice are valuable tools for understanding the diverse pathways affected in the pathogenesis of $\mathrm{AD}$ and for testing therapeutic strategies which could eventually be translated to prevention or treatment of AD in humans.

\section{Neuronal Mitochondria}

\section{Neuronal Energy Production}

The human brain is remarkably energy-dependent, using $20 \%$ of the body's energy while occupying only $2 \%$ of its mass [25]. The high adenosine triphosphate (ATP) demand of electrical signaling in its estimated 86 billion neurons is largely responsible for this energy dependence. There are 16 billion neurons in the cerebral cortex alone, and each cortical neuron uses an average of 4.7 billion ATP molecules per second [26]. Oxidative phosphorylation in the mitochondria using fuel supplied by glucose and ketone bodies is the main source of neuronal ATP [27], and it follows that mitochondria are critical for the survival and proper functioning of neurons. Disorders of mitochondrial function or impairments in glucose and oxygen delivery are especially detrimental to neuronal survival $[28,29]$.

\section{Mitochondrial Biogenesis, Distribution, and Function in Neurons}

Mitochondria are presumed to arise in the cell body of neurons, but in some cases could be synthesized distally in the axon [30]. The majority of mitochondrial proteins are encoded by nuclear DNA 
and synthesized on ribosomes in the cytosol [31, 32]. Mitochondria distribute along axons and dendrites of neurons, with differences in morphology and spacing between the two compartments [33, 34]. Dendrites are more densely populated, with longer mitochondria, and axons are more sparsely populated with shorter mitochondria. There does not appear to be a bias for mitochondrial localization at synapses in general [33], but there does appear to be activity-dependent distribution [35]. One can imagine how such distribution would be beneficial given the functions of neuronal mitochondria. In the distal neurites, mitochondria produce ATP which acts locally to power ion pumps that maintain membrane potential and counteract synaptic ion fluxes [27]. Mitochondrial ATP also helps neurons meet the anabolic demand of shaping synapses [36]. In addition to producing ATP, mitochondria take part in calcium homeostasis [37] and apoptosis signaling [38]. Mitochondria can also tether to the endoplasmic reticulum where the two organelles signal to one another, but the consequences of such signaling and the pathways involved are only beginning to be unraveled [39].

\section{Mitochondrial Dynamics}

Neuronal mitochondria are dynamic organelles that are transported along the cytoskeleton of neurites in anterograde and retrograde directions [33, 40-43]. The average speed of motile mitochondria in neurons is reported to vary from $0.32-0.91 \mu \mathrm{m} / \mathrm{sec}$ [44], with roughly one third of mitochondria moving at any time and the rest stationary $[33,45]$. Traditional motor proteins, dynein and kinesin, drive mitochondrial movement over long distances in the cell but depend on mitochondria-specific adaptor

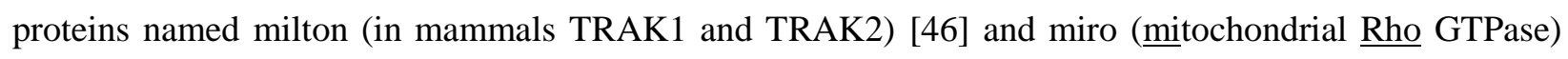
[47] to bind to mitochondria. Miro is anchored to the mitochondrial outer membrane, while TRAK1/2 link miro to the motor proteins [44]. The majority of long-distance mitochondrial trafficking is facilitated by microtubules, but thin actin filaments may enable local trafficking [48]. Stationary mitochondria are anchored to the cytoskeleton [49-52] and a putative axonal docking protein, syntaphilin, was recently described . Expression of syntaphilin promotes a higher percentage of stationary mitochondria, whereas knockdown of syntaphilin enhanced mitochondrial motility in axons [53]. 
In addition to movement along the cytoskeleton, mitochondria undergo fission and fusion. Fusion of the outer membrane requires mitofusins 1 and 2 (MFN1 and MFN2) [54, 55] and OPA1 allows fusion of the inner membrane [56]. A dynamin-related protein, DRP1, is largely responsible for mitochondrial fission [57]. DRP1 forms a constricting spiral upon activation which pinches off the inner and outer mitochondrial membrane [58]. Another outer membrane protein, Fis1, is thought to be involved in recruitment of DRP1, but Fis1 is not necessary for fission [59]. The purpose of mitochondrial fission and fusion is debated, but it is hypothesized that fusion, followed by redistribution of proteins and other membrane components, and then asymmetric fission, leads to replenishment of one mitochondrion at the expense of the slightly more depolarized sister mitochondrion that is later degraded [60].

\section{Mitochondrial Degradation}

Mitochondria experience constant turnover, with a measured in-vivo half-life of 24.4 days for rat brain mitochondria, independent of age [61]. Because neurons are mostly non-dividing cells and mitochondria become damaged over time, mechanisms must exist for damaged mitochondria to be removed to prevent accumulation of reactive oxygen species (ROS) [62] which cause neuronal insult. Mitophagy is a mitochondrially-directed autophagocytic process which removes damaged mitochondria via lysosomal degradation. As would be expected, pharmacological insult of mitochondria in cultured cortical neurons leads to upregulation in mitophagy [63]. Like biogenesis, mitophagy is presumed to occur in the cell body, but a notable exception has been observed in distal axons of cultured neurons [64].

In summary, the cycle of mitochondrial synthesis, anterograde translocation, arrest, retrograde translocation, fission, fusion, and degradation is central to neuronal survival and plasticity. Disruption of this balance is likely a key factor in neurodegeneration including in AD.

\section{Mitochondrial Dysfunction in Alzheimer's Disease}

Mitochondrial dysfunction, which is a known consequence of many cellular insults, is increasingly recognized as a critical step in the pathological cascade leading to the AD phenotype [17, 65]. AD patients show reductions in cerebral glucose metabolism in areas associated with AD pathology 
including hippocampus, parietotemporal cortex, and cingulate cortex years before the onset of $\mathrm{AD}$ symptoms by FDG-PET imaging [3, 66]. Homologous regional alterations in brain metabolism are seen early in life in the triple-transgenic Alzheimer's disease (3xTg-AD) mouse model of AD, and by 18 months all brain areas show reduced glucose metabolism [67]. Human AD patients also show alterations in TCA cycle enzymes in the brain including reduced activity of pyruvate dehydrogenase, isocitrate dehydrogenase, and alpha-ketoglutarate dehydrogenase and increased activity of succinate dehydrogenase and malate dehydrogenase $[68,69]$. These alterations correlate strongly with the clinical stage of disease. The 3xTg-AD mouse similarly shows reduced pyruvate dehydrogenase protein levels and mitochondrial respiration in the brain as early as 3 months of age and reduction in mitochondrial respiration of embryonic primary hippocampal neurons [70]. AD patients are frequently found to have mtDNA mutations or deletions [71], and cybrid studies where mtDNA from peripheral cells of AD patients is expressed in mtDNA-depleted cultured cells demonstrate bioenergetic deficits in the AD cybrid cells [72]. Mitochondrial dysfunction is also seen in the only nontransgenic AD rodent model, where streptozocin injected intracerebrovascularly promotes neuroinflammation, amyloid-beta accumulation, and cognitive changes $[73,74]$.

Mitochondrial movement could also be affected in AD. Abnormalities in axonal transport and axonal swellings are observed early in AD in humans and mouse models [75], implying that mitochondria and other organelles may not be able to distribute normally in those neurons. We and others previously showed that exogenously applied A $\beta$ oligomers can impair mitochondrial movement and cause a reduction in mitochondrial size in primary hippocampal neurons [18-20] and more recently that mitochondrial movement was greatly impaired in 3xTg-AD primary cortical neurons [Cavendish et. al; Article Accepted, Awaiting Publication].

Mitochondria may also be structurally abnormal even before neurodegeneration is evident. Two studies of $\mathrm{AD}$ patients showed reduction in number of neuronal mitochondria $[14,76]$. Changes to neuronal mitochondrial structure, including reduced mitochondrial size, were seen in the hippocampus, auditory cortex, and frontal cortex, as well as several other non-AD associated brain areas of AD patients 
[76]. Abnormal mitochondrial structure, including paracrystalline inclusions between mitochondrial cristae and increased matrix density, was also seen in dendrites of neurons in the frontal cortex of $\mathrm{AD}$ patients [77]. Interestingly, the presence of abnormal mitochondria in this study coincided with cortical areas with more severe dendritic degeneration. One study described a unique mitochondrial structural phenotype resembling "mitochondria on a string" in the hippocampus and entorhinal cortex of human AD patients as well as in the hippocampus of various strains of transgenic AD mice [78]. An in vivo study of mitochondrial structure in APP/PS1 mice showed reduced mitochondrial density and abnormal mitochondrial structure in the immediate vicinity of amyloid plaques [79].

$\mathrm{AD}$-associated mitochondrial deficits are often attributed to the mito-toxic effects of beta amyloid [80-84], yet, as mentioned, amyloid-based treatments have thus far been unsuccessful in ameliorating the symptoms or progression of $\mathrm{AD}$ in humans. One probable reason for the failure of these strategies is that treatment begins too late in the progression of the disease, when significant neuronal damage has already occurred. Another potential reason is that beta amyloid accumulation is only part of a cascade of events leading to mitochondrial damage, which manifests as reduced bioenergetic capacity, impaired mitochondrial dynamics, elevated reactive oxygen species, synaptic dysfunction and loss, and increased apoptosis of $\mathrm{AD}$ neurons. While beta amyloid may indeed initiate this cascade, the effects of impaired or damaged mitochondria could positively feedback to invoke even more mitochondrial damage, leading to a vicious cycle. It is likely that these mitochondria-mediated stresses are both necessary and sufficient to cause neurodegeneration, and $\mathrm{AD}$ treatments should therefore aim to protect and restore mitochondrial function in addition to alleviating other known pathways of neuronal damage. Removing beta amyloid or blocking its effects would only be efficacious before significant mitochondrial damage has occurred.

In addition to alterations of levels and activity of mitochondrial enzymes, changes to mitochondrial structure, and impaired mitochondrial dynamics, many other mechanisms may underlie AD-associated metabolic deficits. Studying mitochondrial structure, number, distribution, dynamics, and function in $\mathrm{AD}$ neurons both in vitro and in $\mathrm{AD}$ animal models can provide more insight into multiple 
mechanistic pathways and lead to new treatment strategies to protect against mitochondrial damage in the early stages of $\mathrm{AD}$ and restore mitochondrial function in advanced $\mathrm{AD}$ patients.

\section{Sigma-1 Receptors for treatment of Alzheimer's Disease}

Compounds which act to prevent or counteract mitochondrial deficits could be useful tools to protect neurons from the irreversible damage that occurs in the progression of AD. Sigma-1 receptor $(\sigma 1 \mathrm{R})$ agonists are a promising drug class to achieve enhanced mitochondrial function among other neuroprotective effects [85]. The $\sigma 1 \mathrm{R}$ is a chaperone protein present in neurons within mitochondrial, nuclear, and plasma membranes. In the inactive state, $\sigma 1 \mathrm{Rs}$ are found in the mitochondrial associated membrane (MAM) of the endoplasmic reticulum (ER) bound to the heat shock 70 protein BiP [38]. Once activated, $\sigma 1$ Rs dissociate from BiP and increase Ca 2+ efflux from the ER through IP3 receptors at the MAM $[86,87]$. Mitochondria then take up Ca 2+ leading to activation of tricarboxylic acid cycle enzymes and enhancement of oxidative phosphorylation.

Although exogenous $\sigma 1 \mathrm{R}$ ligands are not related structurally, several have been identified as selective, high affinity $\sigma 1 \mathrm{R}$ agonists, such as (+)SKF-10,047 [(+)-N-Allylnormetazocine hydrochloride], a member of the benzomorphan drug class. $\sigma 1 \mathrm{R}$ agonists have shown promise for treatment of $\mathrm{AD}$ in preclinical studies [88-90], but whether they exert these effects through mitochondrial mechanisms remains to be fully elucidated. In one of the studies described here, we provide evidence that a $\sigma 1 \mathrm{R}$ agonist can indeed partially restore mitochondrial function in an in vitro model of AD. ANAVEX 2-73, a sigma agonist, is currently in phase II of a clinical trial for treatment of $\mathrm{AD}$, and more compounds are continually being developed which may counteract various aspects of $\mathrm{AD}$.

Other mechanisms of $\sigma 1 \mathrm{R}$ neuroprotection in $\mathrm{AD}$ include attenuation of ROS production $[89,90]$, inhibition of ER stress [87, 91], protection from apoptosis [90, 92], reduction in inflammatory activation of glia [93], and prevention of tau hyperphosphorylation via glycogen synthase kinase $3 \beta$ (GSK3 $\beta$ ) [94]. All of these factors make $\sigma 1 \mathrm{R}$ agonists promising candidates for treating multiple aspects of $\mathrm{AD}$ pathology and warrant further in vivo and clinical study of these compounds. 


\section{Gaps in Knowledge}

There are many unknown aspects of the pathophysiology of AD. Of the many hypotheses explaining the initiating factors and key steps in the development of AD pathology, none have successfully explained all aspects of the disease. The amyloid hypothesis may account for familial AD, but falls short in sporadic AD where it fails to explain why some individuals who exhibit extensive AD pathology have clinically normal cognition, or why some individuals with all of the clinical symptoms of AD show little to no AD pathology [95]. The mitochondrial cascade hypothesis proposes that major differences exist between the pathophysiology of familial $\mathrm{AD}$ versus sporadic $\mathrm{AD}$, and that in sporadic $\mathrm{AD}, \mathrm{A} \beta$ is more of a marker of brain aging than the initial driving factor of disease [17]. This hypothesis, however, does not explain why AD does not occur concurrently with every mitochondrial or metabolic disease. Tau-centric hypotheses propose that tau hyperphosphorylation and aggregation into fibrils is the driving force of $\mathrm{AD}$ pathology and is the final common pathway of a variety of insults, including ROS, heavy metal toxicity, $\mathrm{A} \beta$ oligomers, and cholesterol/LDL dysregulation, which activate microglia to damage neurons and synapses [96]. Tau hypotheses also fall short of encapsulating the full explanation for $\mathrm{AD}$, as not all tauopathies lead to $\mathrm{AD}$ clinical symptoms or amyloid pathology, such as frontotemporal dementia with mutant tau.

One explanation for the lack of any single hypothesis to explain AD pathophysiology is that familial $\mathrm{AD}$ has a separate etiology from sporadic $\mathrm{AD}$, and sporadic $\mathrm{AD}$ could actually describe several related multifactorial diseases with similar outcomes [97]. There is evidence for this in the fact that AD biomarkers exhibit several different profiles with distinct clinical features. For example, AD cases having low $\mathrm{A} \beta_{1-42}$ in CSF and presence of the APOE $\varepsilon 4$ allele (AELO) have later onset than AD with low A $\beta_{1-42}$ and high tau in CSF (ATEO) [97]. More research is needed in understanding the heterogeneity of sporadic $\mathrm{AD}$, and perhaps with improved biomarkers and imaging techniques combined with longitudinal studies of large patient populations even more subtypes of AD might be identified. 
Another issue in the field of $\mathrm{AD}$ research is the overall failure of putative $\mathrm{AD}$ treatments in humans. Currently, the only approved drugs for $\mathrm{AD}$ are the cholinesterase inhibitors donepezil, rivastigmine, and galantamine, and the NMDA receptor antagonist memantine. These medications only improve symptoms of $\mathrm{AD}$, but do nothing to slow or stop the ultimate progression of the disease. There have been over 400 clinical trials for AD drugs, but nearly all have ultimately failed to make an impact on AD progression. As of 2018, there were 112 compounds in AD clinical trials: 26 in phase III, 62 in phase II, and 23 in phase I [98]. Of the phase III trials for disease modifying agents, nearly all are amyloidbased treatments due to the historical focus on $\mathrm{A} \beta$ as the main driver of $\mathrm{AD}$ pathology. Phase I and II trials contain a wider variety of agents, including compounds with anti-inflammatory, metabolic modifying, neuroprotective, and neuroregenerative actions.

While the future of $\mathrm{AD}$ clinical trials appears more promising than the past, problems and questions still exist with the development of $\mathrm{AD}$ therapeutics in general. A major concern of past and current trials is that by the time treatment begins in symptomatic AD patients, it is likely that the disease has been ravaging the brain for many years and that significant, irreversible loss of neurons and synapses has already occurred. AD-associated vascular damage may further complicate delivery of therapeutics, as preliminary data from our lab has shown an extensive and selective loss of small diameter vessels in 3xTg-AD mice (Quintana et. al; publication in revision) and AD patients often have cerebral amyloid angiopathy or other concurrent neurovascular damage. The multifactorial nature of $\mathrm{AD}$ also makes it unlikely that any monotherapy targeting one specific pathway can fully treat all aspects of AD pathology [97]. Earlier identification of individuals at high risk of developing AD combined with more chronic administration of drugs could be beneficial, but these types of trials present more risk of harm to otherwise healthy patients, high costs, and high turnaround time until results are known. It has also been proposed that strategies using pharmaceutical agents targeting multiple pathways, using multiple drugs in combination, and/or stratification of patients into different subtypes of AD could all help overcome the barriers to AD treatment [99]. 


\section{Summary and Conclusions}

With the ever-growing population of AD patients, the substantial reduction of their quality of life and years of life lived, and increasing burden on caregivers and healthcare providers, AD is becoming an increasingly harrowing problem requiring more research to understand its causes and develop diseasemodifying agents. New approaches informed by the mistakes of the past decades are needed which acknowledge the multifactorial and heterogeneous nature of the disease as well as the potential that the pathological changes leading to $\mathrm{AD}$ often begin many years before clinical symptoms are apparent.

Metabolic and mitochondrial abnormalities are among the earliest changes in $\mathrm{AD}$, and there is significant evidence that mitochondrial damage is a critical step in AD progression. Transgenic mice and in vitro models of $\mathrm{AD}$ provide robust opportunities for studying the diverse changes to mitochondrial biogenesis, structure, dynamics, and function in the pathogenesis of AD. They also allow testing of compounds for ameliorating AD pathology via modulation of mitochondria. Translational research of mitochondria in AD can help the development of compounds that can be used alone or as part of multidrug regimens in the prevention and treatment of $\mathrm{AD}$.

The purpose of the work in this dissertation was to understand the very late and very early mitochondrial changes in AD using a mouse model of the disease. I examined mitochondrial morphology, number, and movement in embryonic cortical neurons from 3xTg-AD mice to understand if mitochondrial changes occurred at the very earliest stage of potential disease, during neuronal development, and see if mitochondrial deficits were responsive to treatment with a $\sigma 1 \mathrm{R}$ agonist. I also performed a large-scale microscopic analysis of mitochondria in various brain areas of aged 3xTg-AD mice at the latest stages of $\mathrm{AD}$ to identify the pathological correlates of mitochondrial morphology, number, and distribution. Together these aims helped to inform the mitochondrial aspects of AD and evaluate the potential for early amelioration of $\mathrm{AD}$ mitochondrial pathology in at-risk populations and enhancement of mitochondrial function late in disease progression. 


\section{Chapter 2}

\section{Mitochondrial Movement and Number Deficits in Embryonic Cortical Neurons from 3xTg-AD Mice}

John Z. Cavendish, Saumyendra N. Sarkar, Mark A. Colantonio, Dominic D. Quintana, Nadia Ahmed, Brishti A. White, Elizabeth B. Engler-Chiurazzi and James W. Simpkins

Cavendish, JZ. Sarkar, SN. Colantonio, MA. Quintana, DD. Ahmed, N. White, BA. Engler-Chiurazzi, EB, Simpkins, JW. 2019. Mitochondrial movement and number deficits in embryonic cortical neurons from 3xTg-AD mice. Journal of Alzheimer's Disease. 10.3233/JAD-190143. (Accepted). 


\section{ABSTRACT}

Mitochondrial dysfunction is often found in Alzheimer's Disease (AD) patients and animal models. Clinical severity of AD is linked to early deficiencies in cognitive function and brain metabolism, indicating that pathological changes may begin early in life. Previous studies showed decreased mitochondrial function in primary hippocampal neurons from triple-transgenic Alzheimer's disease (3xTg-AD) mice and mitochondrial movement and structure deficits in primary neurons exposed to amyloid beta oligomers. The present study characterized mitochondrial movement, number, and structure in 3xTg-AD primary cortical neurons and nontransgenic (nonTg) controls. We found a significant reduction in mitochondrial number and movement in 3xTg-AD primary cortical neurons with modest structural changes. Additionally, application of the sigma-1 receptor agonist, (+)SKF-10,047, markedly increased mitochondrial movement in both 3xTg-AD and nonTg primary cortical cultures after one hour of treatment. (+)SKF-10,047 also led to a trend of increased mitochondrial number in 3xTg-AD cultures. Embryonic mitochondrial movement and number deficits could be among the key steps in the early pathogenesis of $\mathrm{AD}$ that compromise cognitive or metabolic reserve, and amelioration of these deficits could be a promising area for further preclinical and clinical study.

Keywords: Alzheimer's Disease, Mitochondrial Size, Mitochondrial Dynamics, Sigma Receptors 


\section{INTRODUCTION}

Alzheimer's disease (AD) is characterized by a progressive decline in cognition, atrophy of cerebral cortex and hippocampus, loss of synapses, and disruption of neuromodulatory systems [11, 100]. Histopathological hallmarks of AD include amyloid-beta $(\mathrm{A} \beta)$ peptide aggregates and hyperphosphorylated tau-related neurofibrillary tangles, but the contribution of these neuropathological features to the emergence of $\mathrm{AD}$ symptoms, especially cognitive decline, is still unclear [101]. Growing evidence suggests that mitochondrial dysfunction is a critical step in the pathological cascade leading to the $\mathrm{AD}$ phenotype $[17,102]$. AD patients and at-risk individuals show early regional hypometabolism throughout the cerebral cortex and hippocampus [3]. Mitochondrial structural abnormalities including reduced mitochondrial size have also been reported in brains of AD patients [76, 77] and mouse models [78]. Reduced levels and activity of tricarboxylic acid cycle enzymes in AD brain tissue have been reported [69], and there is a reduction in mitochondrial number in pyramidal neurons in $\mathrm{AD}$ patients, corresponding to an increase in mitochondrial DNA within autophagocytic cytoplasmic granules [14]. Reduced mitochondrial number was also seen in M17 cells overexpressing wild-type or mutant APP [103]. Further, AD patients often harbor mitochondrial DNA mutations or deletions [104].

The triple transgenic Alzheimer's disease (3xTg-AD) mouse has three mutations

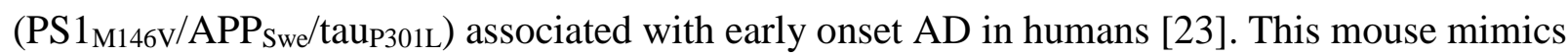
the human $\mathrm{AD}$ phenotype, with progressive $\mathrm{A} \beta$ production, tau hyperphosphorylation, and neurofibrillary tangle formation across the cortex and hippocampus as well as accelerated cognitive decline [24]. Like human patients, 3xTg-AD mice exhibit early bioenergetic deficits, including reduced ATP production in embryonic hippocampal neurons [70]. We and others 
previously showed that exogenously applied $A \beta$ oligomers can impair mitochondrial movement and cause a reduction in mitochondrial size in primary hippocampal neurons [18-20].

$3 x \operatorname{Tg}-\mathrm{AD}$ primary cortical neurons express detectable levels of $\mathrm{A} \beta$ as early as 7 days in vitro (DIV), and $A \beta$ increases with time in culture [105]. Prior to this study, it was not known whether the early expression of $A \beta$ in 3xTg-AD neurons would cause the same mitochondrial phenotypes seen in primary neurons exposed to exogenous $A \beta$. To probe this question, we measured mitochondrial structure, number, and movement in primary cortical neurons from 3xTg-AD mice and non-transgenic (nonTg) controls. We hypothesized that 3xTg-AD neurons would exhibit impairment in mitochondrial movement along neurites, reduced mitochondrial size, and increased roundness compared to nonTg mice. We also hypothesized that there would be decreased mitochondrial number in $3 \times \mathrm{Tg}$ - $\mathrm{AD}$ neurons similar to the reduction observed in human AD patients [14].

Compounds which act to prevent or counteract mitochondrial deficits could be useful tools to protect neurons from the irreversible damage that occurs in the progression of AD. Sigma-1 receptor $\left(\sigma_{1} \mathrm{R}\right)$ agonists are a promising drug class to achieve enhanced mitochondrial function among other neuroprotective effects [85]. The $\sigma_{1} \mathrm{R}$ is a chaperone protein present in neurons within mitochondrial, nuclear, and plasma membranes. In the inactive state, $\sigma_{1} R s$ are found in the mitochondrial associated membrane (MAM) of the endoplasmic reticulum (ER) bound to the heat shock 70 protein $\mathrm{BiP}$ [106]. Once activated, $\sigma_{1}$ Rs dissociate from $\mathrm{BiP}$ and increase $\mathrm{Ca}^{2+}$ efflux from the ER through IP3 receptors at the MAM [86, 87]. Mitochondria then take up $\mathrm{Ca}^{2+}$ leading to activation of tricarboxylic acid cycle enzymes and enhancement of oxidative phosphorylation. Although exogenous $\sigma_{1} \mathrm{R}$ ligands are not related structurally, several have been identified as selective, high affinity $\sigma_{1} \mathrm{R}$ agonists, such as (+)SKF-10,047 [(+)-N- 
Allylnormetazocine hydrochloride], a member of the benzomorphan drug class. $\sigma_{1} \mathrm{R}$ agonists have shown promise for treatment of AD in preclinical studies [88-90], but whether they exert these effects through mitochondrial mechanisms remains to be fully elucidated.

To gain more insight into this question, we studied the effects of $\sigma_{1} R$ activation on mitochondrial structure, number, and movement in primary cortical neurons from 3xTg-AD and nonTg mice using (+)SKF-10,047. Movement of mitochondria along microtubules is carried out through kinesin and dynein proteins, which require ATP for their function [107]. Decreased ATP production, as seen in $\mathrm{AD}$ neurons, could impair mitochondrial movement. $\sigma_{1} \mathrm{R}$ activation can increase oxidative phosphorylation, which could provide added energy for mitochondrial movement. Therefore, we hypothesized that (+)SKF-10,047 would increase mitochondrial movement in primary cortical neurons. Additionally, $\sigma_{1} \mathrm{R}$ agonists can protect against the intracellular stressors that lead to mitochondrial fragmentation and reduced mitochondrial number through multiple downstream effects of $\sigma_{1} R$ activation [108]. We hypothesized that (+)SKF-10,047 would increase mitochondrial size and number in primary cortical neurons while reducing mitochondrial roundness.

\section{MATERIALS AND METHODS}

Mice

Animal protocols were performed in accordance with the National Institutes of Health Guide for the Care and Use of Laboratory Animals and were approved by the Institutional Animal Care and Use Committee of West Virginia University. Homozygous 3xTg-AD and nonTg controls (C57BL6/129S) were obtained from The Jackson Laboratory, and colonies were 
bred and maintained at West Virginia University. Animals were housed in cages of up to five animals on a 12-hour light cycle with ad libitum access to food and water.

\section{Primary cortical culture}

Pregnant mice were anesthetized with isoflurane and euthanized by cervical dislocation at E18.5. Brains of pups were removed and placed in ice-cold Hank's Balanced Salt Solution (HBSS). Cortices were dissected and meninges were removed with fine forceps before transferring to neurobasal culture medium (Gibco) supplemented with 2\% B-27 (Gibco), 1\% pen-strep (HyClone), and 1\% Glutamax (Gibco). Cortices were triturated 10-15 times with a 5 $\mathrm{mL}$ pipette followed by a series of three flame-polished Pasteur pipettes with increasingly narrow tip diameter before being filtered through a $70 \mu$ m nylon cell strainer (Falcon). Cells were plated on poly-L-lysine (Sigma) coated $35 \mathrm{~mm}$ glass-bottom dishes (glass diameter 23mm, thickness $0.17 \mathrm{~mm}$; World Precision Instruments) at a density of $7.5 \times 10^{5}$ cells in $2 \mathrm{~mL}$ of medium and maintained in a $95 \%$ humidity, $5 \% \mathrm{CO}_{2}, 37^{\circ} \mathrm{C}$ incubator for up to two weeks. At 2 DIV, 1-beta-D-arabinofuranosylcytosine (AraC; Sigma) was added at a final concentration of 4 $\mu \mathrm{mol} / \mathrm{L}$ to inhibit glial growth. Subsequently, half of the culture medium was replaced every three days.

\section{Mitochondrial labeling}

\section{$\underline{\text { Mitotracker® Red CMXRos }}$}

Immediately prior to imaging, media was replaced with $80 \mathrm{nmol} / \mathrm{L}$ Mitotracker ${ }^{\circledR}$ Red CMXRos (MT-Red; Thermo Fisher Scientific) in complete neurobasal media and incubated for 15 min at $37^{\circ} \mathrm{C}$. MT-Red solution was then removed and replaced with the culture-conditioned media in which cells had been growing prior to staining. 


\section{pDsRed2-Mito vector}

pDsRed2-Mito vector (Clontech) was amplified using GT115 competent E. coli cells (InvivoGen) and isolated with a Midiprep kit (Thermo Fisher Scientific). For each transfection, 8 $\mu \mathrm{g}$ of plasmid DNA was diluted in $100 \mu \mathrm{L}$ serum-free Opti-MEM I (Thermo Fisher Scientific). $10 \mu \mathrm{L}$ Lipofectamine-2000 (Thermo Fisher Scientific) was diluted in $100 \mu \mathrm{L}$ serum-free Opti MEM I and allowed to stand for $10 \mathrm{~min}$ at room temperature. The plasmid and lipofectamine solutions were combined, gently mixed, and allowed to stand for $20 \mathrm{~min}$ at room temperature before application to cultures. Cells were transfected at 12.5 DIV. Cultures were imaged 36 hours post-transfection.

Preparation of $(+) S K F-10,047$

(+)SKF-10,047 (Tocris) was dissolved in sterile milliQ water to create a 10mM stock solution which was aliquoted and stored at $-20^{\circ} \mathrm{C}$. Just prior to application to cells, a $2 \mu \mathrm{mol} / \mathrm{L}$ working solution was made by diluting the stock solution 1:5000 in pre-warmed complete neurobasal media. Vehicle controls (Veh) were made by diluting sterile milliQ water 1:5000 in pre-warmed complete neurobasal media.

\section{Live-cell imaging}

The investigator performing imaging experiments was blinded to treatment group (Veh or SKF). Widefield imaging was performed on a Nikon Eclipse TE2000-E microscope with an oil immersion 60x Apochromat DIC objective (Nikon) with 1.49 numerical aperture. Neither a polarizer nor an analyzer was used. The microscope's $1.5 \mathrm{x}$ magnification lens was used to give a final magnification of 90x. A halogen light source was used with an ND4 neutral density filter, an ET555/25x excitation filter, and an ET605/52m emission filter (Chroma Technology Corp.) to 
image mitochondria stained with MT-Red or pDsRed2-Mito vector. For comparisons of 3xTg$\mathrm{AD}$ and nonTg cultures at 12-14 DIV, fluorescence images were captured with an exposure time of 60ms (MT-Red-stained cultures) or 300ms (pDsRed2-Mito-stained cultures) on a CoolSNAP monochrome CCD camera (Photometrics) with 2x2 binning and 1024x1024 resolution giving a pixel size of $141 \mathrm{~nm}$. For experiments at 7-9 DIV, fluorescence images were captured with an exposure time of $60 \mathrm{~ms}$ on an ORCA CMOS camera (Hamamatsu) with 2x2 binning and $2048 \times 2048$ resolution giving a pixel size of $72 \mathrm{~nm}$. During imaging, cultures were maintained in a humidified digital stage-top incubator (OKO Labs) at $37^{\circ} \mathrm{C}$ and $5 \% \mathrm{CO}_{2}$.

\section{Imaging of DIV12-14 cultures}

For structural imaging of MT-Red-stained DIV12-14 cultures, eight single-plane images of $143.36 \mu \mathrm{m}$ x $143.36 \mu \mathrm{m}$ were captured at equidistant points along a circle midway between the center of the culture and the culture edge. For DIV14 pDsRed2-Mito-transfected cultures, an individual transfected neuron was selected. Time-lapse images from five non-overlapping fields of view containing separate proximal neurites at varying distances within 10-100 $\mu \mathrm{m}$ of the cell body were acquired every 15 seconds for one hour.

\section{Imaging of DIV7-9 cultures and treatment with $(+)$ SKF-10,047}

For DIV7-9 cultures, time-lapse images were acquired at five non-overlapping fields of view approximately $250 \mu \mathrm{m}$ apart every 30 seconds for 30 minutes starting at a point halfway between the center of the culture and the culture edge. Fifteen single-plane images were then captured along a circle halfway between the edge and center of culture. Half of the culture medium was then replaced with vehicle or (+)SKF-10,047-containing medium to achieve a final concentration of $1 \mu \mathrm{mol} / \mathrm{L}$. We chose this concentration based on the $\mathrm{EC}_{50}$ value of $0.8 \mu \mathrm{mol} / \mathrm{L}$ (+)SKF-10,047 previously reported for in vitro studies of neuroprotection [109]. One hour post- 
treatment, fifteen more single-plane images were captured halfway between the center of the culture and the culture edge at points that had not previously been imaged. Finally, a 30-minute post-treatment time-lapse series was captured in the same way as pre-treatment comprising five fields of view that had not been previously imaged separated by approximately $250 \mu \mathrm{m}$.

Image analysis

Investigators performing data analysis were blinded to treatment group and genotype.

Mitochondrial structure and number

Images were exported from their native ND2 formats to 16-bit TIFF files using NIS elements (Nikon). Mitochondrial size, width, and number were calculated using an automated Matlab program generously provided by P.M. McClatchey which has been previously described [110] (Supplementary Figure 2.1A). Mitochondrial roundness (inverse of aspect ratio) was calculated by dividing width by height, with a value of 1 corresponding to a perfect circle and 0 corresponding to an infinitely long polygon in 1 dimension. A total of four fields of view per culture were used for DIV12-14 cultures and eight fields of view were used for DIV7-9 cultures. Odd numbered images were selected for analysis unless they contained putative glial cells, as identified by lack of neurites and a reticular network of mitochondria (Supplementary Figure 2.1B). If a glial cell was present, the closest even-numbered image was used for analysis. Individual mitochondrial measures from all four (DIV12-14) or eight (DIV7-9) fields of view of a culture were combined and averaged to give average length, width, and roundness for that culture. The total number of mitochondria was divided by the combined imaging area to yield number of mitochondria per square micron. Each culture was treated as an independent $\mathrm{n}$.

\section{$\underline{\text { Mitochondrial movement analysis in pDsRed2-Mito-transfected neurons }}$}


Analysis was carried out in NIS elements (Nikon). Time-lapse images were aligned, and the advanced de-noising function was used with de-noising power of 50 followed by rolling ball background subtraction (radius $=1 \mu \mathrm{m}$ ). Five $1.1 \mu \mathrm{m}$ by $6 \mu \mathrm{m}$ regions of interest (ROIs) were placed orthogonal to proximal neurites between $10 \mu \mathrm{m}$ and $100 \mu \mathrm{m}$ from the cell body. The ROI width was chosen based on a previously described method for tracking mitochondrial occupancy along neurites [33]. A time measurement of maximum fluorescence intensity within ROIs was then performed. Only the first 15 minutes of imaging were included to minimize potential phototoxicity effects. Mitochondrial movement events were defined as an increase or decrease of maximum fluorescence intensity of at least 200 fluorescence units over 30 seconds or less, corresponding to mitochondrial movement into or out of the ROI (Supplementary Figure 2.2A). Mitochondrial transit events, defined as a movement event in which a mitochondrion passed completely from one side of the ROI to the other, were manually counted (Supplementary Figure 2.2B).

$\underline{\text { Mitochondrial movement analysis in Mitotracker® Red CMXRos-stained neurons }}$

Time-lapse images were aligned in NIS elements and exported as stacks of 16-bit TIFF files. Despeckling, background subtraction (rolling ball method; radius $=20$ pixels), and autothresholding using the Otsu method were performed in FIJI (NIH) to create binary masks. One 300 pixel by 300 pixel $(21.6 \mu \mathrm{m}$ x $21.6 \mu \mathrm{m})$ subregion was randomly generated within each image using a random number generator in Excel (Microsoft) for $\mathrm{x}$ and $\mathrm{y}$ coordinates. If the subregion contained part of a cell body or out-of-focus material, a new region was randomly generated until only in-focus neurites were found within the region. The subregion was cropped and imported into Imaris (Bitplane). Manual spot tracking of every mitochondrion present within the subregion for at least two frames $(1 \mathrm{~min})$ was performed. Tracking consisted of placing a dot 
at the object's center of mass starting at the first frame where it was visible until it (1) disappeared from the edge of the field of view; (2) disappeared for more than 3 consecutive frames without reappearing in the same location during that time; or (3) fused with another object for at least 3 consecutive frames. In the case of fusions, the larger of the two objects continued to be tracked while the smaller object stopped being tracked at the time of fusion. If an object split from another object for at least 3 frames, it was counted as a new object and tracked from the first frame of clear separation from the other object. After all objects were tracked, measurements were exported and average velocity of each spot was calculated by dividing total displacement by track duration. One subregion was tracked in each field of view, and three fields of view were analyzed for each culture. All objects' velocities from the three fields of view were pooled and averaged to give an average mitochondrial velocity for each culture.

\section{Statistical analysis}

All statistical analyses were performed using Prism version 7.04 (GraphPad). Two-tailed unpaired t-tests were used to assess the effect of genotype on mean mitochondrial length, width, roundness, number $/ \mu \mathrm{m}^{2}$, movement events/min, transits/min, and velocity between nonTg and $3 x T g-A D$ cultures with a significance level of $p<0.05$. For structure and number measurements in DIV12-14 cultures, $n=11$ nonTg and $n=113 x T g-A D$ cultures spread across three separate pregnancies each were compared. For movement measures in DIV14 cultures, $\mathrm{n}=8$ nonTg and $\mathrm{n}=73 \mathrm{xTg}-\mathrm{AD}$ cultures (one neuron per culture) spread across three pregnancies each were compared. For structure, number, and movement measures in DIV7-9 cultures, pre-treatment values from $n=6$ nonTg and $n=63 x T g-A D$ cultures from one pregnancy each were compared. Two-way analysis of variance (ANOVA) was used to assess effect of genotype (nonTg vs. 3xTgAD) and treatment (Vehicle or (+)SKF-10,047) on mitochondrial structure, number, and velocity 
one hour post-treatment in DIV7-9 cultures with a significance level of $p<0.05$. Two-tailed ttests were used to probe effects of treatment within genotypes if significant interaction between genotype and treatment were observed with ANOVA. $n=3$ cultures per group were used for each treatment and genotype and were derived from one pregnancy for each genotype.

\section{RESULTS}

Mitochondrial structure and number in 3xTg-AD cortical neurons at 12-14 DIV.

Within individual cultures, mitochondria ranged widely in shapes and sizes including small spheres, larger ovoid shapes, rods of varying length, and branching filaments (Fig. 2.1A,B). However, average mitochondrial size, number, and roundness remained fairly consistent across different cultures within each group as long as a sufficient number of fields of view were sampled. At 12-14 DIV, mitochondrial length was significantly reduced in 3xTg-AD cultures $(\mathrm{t}(20)=5.108, \mathrm{p}<0.0001)$ (Fig. 2.1C). Mitochondrial width showed a marginal tendency toward being increased in $3 \times \mathrm{Tg}-\mathrm{AD}$ cultures $(\mathrm{t}(20)=2.024, \mathrm{p}=0.0565)$ (Fig. 2.1D). Consistent with these observations, mitochondrial roundness was significantly increased in $3 x T g-A D$ cultures compared to nonTg cultures $(\mathrm{t}(20)=5.568, \mathrm{p}<0.0001)$ (Fig. 2.1E). The most pronounced difference between cultures was a decrease in mitochondrial number in $3 x T g$-AD cultures ( $t(20)$ $=4.226, \mathrm{p}=0.0004)($ Fig. $2.1 \mathrm{~F})$.

Mitochondrial movement in neurites of 3xTg-AD cortical neurons at $14 \mathrm{DIV}$.

Mitochondrial movement was assessed in neurites of pDsRed2-mito transfected neurons at 14 DIV using ROIs placed 10-100 $\mu \mathrm{m}$ from the cell body (Fig 2.2A,B). Movement events were significantly reduced in $3 \times \mathrm{Tg}-\mathrm{AD}$ neurites compared to nonTg controls $(\mathrm{t}(13)=2.942$, 
$\mathrm{p}=0.0115)$ (Fig 2.2C). There was also a reduction in number of passing mitochondria per minute in 3xTg-AD neurites $(\mathrm{t}(13)=3.069, \mathrm{p}=0.0090)($ Fig 2.2D).

Mitochondrial structure and number in 3xTgAD cortical neurons at 7-9 DIV

Mitochondrial number and structure were compared in nonTg and 3xTg-AD neurons at 7-9 DIV (Fig. 2.3A,B). There was no significant difference in mitochondrial length between nonTg and 3xTg-AD cultures (Fig. 2.3C). Mitochondrial width was significantly increased in $3 \mathrm{xTg}-\mathrm{AD}$ neurites at 7-9 DIV $(\mathrm{t}(10)=3.962, \mathrm{p}=0.0027)$ (Fig. 2.3D). Mitochondrial roundness was slightly but significantly decreased in $3 x T g-A D$ cultures $(t(10)=3.609, p=0.0048$ ) (Fig. 2.3E). Similar to DIV12-14 cultures, 3xTg-AD cultures at 7-9 DIV had significantly fewer mitochondria per square micron than nonTg cultures $(\mathrm{t}(10)=9.316, \mathrm{p}<0.0001)$ (Fig. 2.3F). The percent decrease in mitochondrial number in $3 \times \mathrm{Tg}-\mathrm{AD}$ cultures at 7-9 DIV was similar to the change at 12-14 DIV.

Mitochondrial movement in neurites of 3xTg-AD cortical neurons at 7-9 DIV and effect of $(+) S K F-10,047$

Mitochondrial velocity was measured before and one hour post-treatment with vehicle or $1 \mu \mathrm{mol} / \mathrm{L}(+) \mathrm{SKF}-10,047$ in nonTg and 3xTg-AD neurons at 7-9 DIV (Fig. 2.4A). The pretreatment velocity of mitochondria in 3xTg-AD cultures was significantly lower than nonTg cultures $(\mathrm{t}(10)=12.9, \mathrm{p}<0.0001)$ (Fig. 2.4B). Two-way ANOVA revealed a significant effect of both genotype $(F(1,8)=72.8, \mathrm{p}<0.0001)$ and $(+)$ SKF-10,047 treatment $(F(1,8)=20.67$, $\mathrm{p}=0.0019$ ) on mitochondrial velocity in cultured cortical neurons (Fig. 2.4C). There was no significant interaction between the effects. NonTg cultures exhibited markedly higher velocities of mitochondrial movement compared to 3xTgAD cultures under either treatment condition (Fig. 
2.4C; upper inset). Additionally, (+)SKF-10,047-treated cultures exhibited markedly higher velocities of mitochondrial movement compared to vehicle-treated cultures (Fig. 2.4C; lower inset).

Effect of $(+) S K F-10,047$ on mitochondrial structure and number in cortical neurons at 7-9 DIV

Mitochondrial number and structure measurements were taken one hour post-treatment with vehicle or $1 \mu \mathrm{mol} / \mathrm{L}(+)$ SKF-10,047 in nonTg and 3xTg-AD cultures (Supplementary Fig. 2.3A). Two-way ANOVA of mitochondrial length showed a significant interaction between genotype and treatment $(\mathrm{F}(1,8)=7.122, \mathrm{p}=0.0284)$. Probing this significant interaction, $(+)$ SKF10,047 treatment significantly increased length in nonTg cultures $(\mathrm{t}(4)=3.237, \mathrm{p}=0.0318$ ) but not in $3 \times \mathrm{TgAD}$ cultures (Supplementary Fig. 2.3B). There was a main effect of genotype on mitochondrial width $(\mathrm{F}(1,8)=30.25$, $\mathrm{p}=0.0006)$ such that DIV7-9 3xTg-AD cultures were wider than nonTg cultures regardless of treatment condition (Supplementary Fig. 2.3C). There was a main effect of $(+)$ SKF-10,047 treatment on roundness $(F(1,8)=24.54, \mathrm{p}=0.0011)$ such that (+)SKF-10,047 significantly reduced roundness in both nonTg and 3xTg-AD cultures (Supplementary Fig. 2.3D). There was a significant interaction $(F(1,8)=13.4, p=0.0064)$ between genotype and treatment on mitochondrial number. NonTg cultures had no significant change in number after (+)SKF-10,047 while 3xTg-AD cultures trended toward increased number $(\mathrm{t}(4)=2.57, \mathrm{p}=0.0620)$ at one hour post-treatment (Supplementary Fig. 2.3E).

\section{DISCUSSION}

This study demonstrates that $3 \times \mathrm{Tg}-\mathrm{AD}$ primary cortical neurons have reduced mitochondrial number and movement at both 7-9 DIV and 12-14 DIV compared to nonTg controls. This observation adds to growing evidence that mitochondrial abnormalities early in 
life, perhaps even during development, are a key pathological feature of AD. One complication of traditional $\mathrm{A} \beta$ - and phospho-tau-centric hypotheses of $\mathrm{AD}$ pathophysiology is the fact that a subset of people develop AD pathology without cognitive symptoms [6]. It has been proposed that individuals with a more extensive "cognitive reserve" or "metabolic reserve" are resistant to clinical symptoms of $\mathrm{AD}$ in the face of $\mathrm{AD}$ pathology $[111,112]$. These hypotheses focus on environmental influences on setting up cognitive and metabolic reserves, but it is also possible that early developmental factors could be involved.

Reduction in mitochondrial movement was the most pronounced difference between 3xTg-AD primary cortical neurons and nonTg controls. At 12-14 DIV, about half as many mitochondrial movement events occurred in neurites of 3xTg-AD cultures compared to nonTg controls. We also observed a large reduction in average mitochondrial velocity in neurites of 3xTg-AD neurons when examined at 7-9 DIV. Reduction in mitochondrial movement could lead to problems with distribution of mitochondria at proper locations along axons and dendrites, which could negatively impact synaptic transmission [107]. These deficits, over time, could potentially contribute the loss of synapses seen in AD.

Mitochondrial number was also consistently reduced in 3xTg-AD primary cortical neurons, which had about three-fourths the number of mitochondria per square micron of culture compared to nonTg controls. There was not a significant difference in the number of neuronal cell bodies between 3xTg-AD and nonTg cultures at either 7-9 DIV or 12-14 DIV (data not shown). An early reduction in mitochondrial number and/or function could be detrimental to survival and normal differentiation of neurons. Some of the hallmarks of AD, including loss of synapses and reduction in grey matter volume, would be expected consequences of early mitochondrial dysfunction in neurons. Additional studies of changes in mitochondrial number in 
both the 3xTg-AD mouse and human patients at various stages of disease could help to determine if reduced mitochondrial number is a factor in the pathogenesis of AD. Additionally, studies which elucidate the mechanisms responsible for reduced mitochondrial number in $3 \mathrm{xTg}$ AD primary neurons are needed.

Mitochondrial structure also differed between nonTg and 3xTg-AD primary cortical neurons, depending on time in vitro, but these differences were not as marked as differences in movement or number. $3 \times \mathrm{Tg}-\mathrm{AD}$ cultures at 12-14 DIV had significantly shorter and rounder mitochondria. This would be expected given previous observations that exogenous treatment with $A \beta$ oligomers causes reduced mitochondrial length $[18,19]$, although the change in the current study was not as marked as the change with exogenous $A \beta$ application. On the other hand, reduction in mitochondrial length and increased roundness were not present at 7-9 DIV. Because $A \beta$ expression increases with time in culture for 3xTg-AD neurons [105], it is possible that mitochondrial length reduction is dependent on the amount of intracellular $\mathrm{A} \beta$ present. We speculate that mitochondrial structural changes at 7-9 DIV could have been compensatory for the reduction in mitochondrial number.

Reversing mitochondrial abnormalities early in development could be a promising strategy for preventing the cascade of events that leads to clinical dementia later in life, including loss of synapses. The $\sigma_{1} R$ agonist (+)SKF-10,047 significantly increased mitochondrial movement in both nonTg and 3xTg-AD cortical neurons. In 3xTg-AD neurons there was a more than two-fold increase in mitochondrial velocity, which brought the final velocity closer to the baseline nonTg average velocity. The mechanism of this increase is unclear, but it is possible that the enhancement of mitochondrial function caused by sigma- 1 receptor activation $[86,87]$ could be responsible for the increased movement. Additionally, sigma-1 receptors are known to 
translocate from the MAM to other membrane compartments of the cell, including mitochondria, upon stimulation with an agonist. Sigma-1 receptors could potentially interact with proteins within or adjacent to the mitochondrial membrane involved in mitochondrial translocation including dynein, members of the kinesin-1 family, and mitochondrial rho (MIRO) proteins among others. Of these, MIRO1 and MIRO2, which serve as adaptor proteins linking mitochondria to kinesin, are particularly promising candidates. It has been shown that sigma-1 receptors bind to Rac1 GTPase in isolated brain mitochondria with increased binding after stimulation with sigma-1 agonists [113]. Sigma-1 receptor binding to MIRO could enhance movement by stabilizing MIRO-kinesin binding or increasing kinesin activity. Future studies are needed to document this.

(+)SKF-10,047 also led to a trend of increased mitochondrial number in 3xTg-AD neurons at 7-9 DIV after one hour of treatment. This increase occurred too fast to be explained by mitochondrial biogenesis which relies on gene expression from nuclear DNA and protein production in the cytosol [114]. It is possible that reduced mitophagy triggered by sigma-1 receptor activation could lead to increased mitochondrial number, as inhibition or loss of sigma-1 receptors has been shown to increase mitophagy [115]. Perhaps a change in fission/fusion dynamics could be occurring, but this would be expected to cause mitochondrial structure changes; (+)SKF-10,047 had minimal effects on mitochondrial structure in both nonTg and $3 \mathrm{xTg}-\mathrm{AD}$ cortical neurons. One final possibility is that (+)SKF-10,047 increased mitochondrial membrane potential. Because Mitotracker® Red-CMXRos fluorescence intensity depends on mitochondrial membrane potential [116], an increase in mitochondrial membrane potential could cause previously undetected depolarized mitochondria to increase their signal. 
The effects of (+)SKF-10,047 on mitochondrial movement and number observed in primary neurons warrant future studies of the mechanisms of action of sigma-1 receptors in relation to mitochondrial movement, biogenesis, and function. The potential mechanisms of action of (+)SKF-10,047 on increasing mitochondrial movement should be evaluated, including effects on mitochondrial ATP production and interaction with mitochondrial motor proteins. The relationship of reduced mitochondrial number and movement to ER stress should also be examined, as ER stress markers are increased in 3xTg-AD mice as early as 2 months of age [117]. Sigma-1 receptors attenuate ER stress [118] and could potentially exert their mitochondrial effects through this pathway. It should be determined if the effects observed in the current study are limited to cultured primary cortical neurons or if they are seen in other cell types in vitro, such as glia or hippocampal neurons. Further, in vivo studies should be carried out to determine if sigma-1 receptor agonists similarly increase mitochondrial number in neurons in healthy or diseased states and inform their potential use as pharmacological agents to counteract mitochondrial damage associated with neurodegenerative diseases.

Mitochondrial number and movement deficits, such as those seen in 3xTg-AD primary cortical neurons, could be an early initiating factor for synaptic dysfunction and neuronal loss later in life. A caveat of this conclusion is that APP, A $\beta$, and phospho-tau accumulation in the $3 \times \mathrm{Tg}-\mathrm{AD}$ mouse is driven by transgenes which are not present in the vast majority of sporadic AD cases in humans. Other developmental insults to mitochondria, however, may still potentially hinder the establishment of cognitive and metabolic reserves and increase risk of $\mathrm{AD}$ neurodegeneration later in life. For example, germline mitochondrial DNA mutations have been found to cause premature aging and marked cortical and hippocampal abnormalities in adult mice [119]. Our study provides evidence of early mitochondrial abnormalities in a model of 
familial AD. Our findings suggest that early treatment with sigma-1 receptor agonists could help ameliorate $\mathrm{AD}$-associated mitochondrial movement and number deficits at critical stages of neurogenesis and neuronal differentiation.

One limitation of this study is that we did not differentiate between axons and dendrites. Mitochondria are significantly smaller and occupy a lower percentage of axons compared to dendrites [33], and mitochondria move more often and faster in axons compared to dendrites [33, 120]. We attempted to overcome this limitation by sampling an adequate number of fields of view and sampling consistently between different cultures so that an equal proportion of axons and dendrites were represented among samples. Another limitation is the inability to directly compare the cultures at 7-9 DIV and 12-14 DIV. Mitochondria are longer and move less in cultures at higher DIV [121]. There is also a significantly higher density of mitochondria in culture at 12-14 DIV compared to 7-9 DIV. We were able to use MT-Red to analyze structure at both time points, but we could not do individual tracking of mitochondria with MT-Red at 12-14 DIV as we did at 7-9 DIV due to the high number of mitochondria. Therefore we used pDsRed2Mito-transfected cultures for measurement of mitochondrial movement using ROIs. One caveat of this method is that there is a difference in events/min depending on the distance of the ROI from the cell body. To overcome this, we measured ROIs in the same range of distances from the cell body in nonTg and 3xTg-AD neurons.

\section{CONCLUSIONS}

Mitochondrial movement and number deficits were observed in primary cortical neurons derived from embryonic $3 \times \mathrm{Tg}-\mathrm{AD}$ mice. These, along with previously observed early mitochondrial function deficits [70], could be key initiating factors in the development of AD pathology later in life. Early mitochondrial deficits could also predispose individuals to reduced 
cognitive or metabolic reserve, which relate to clinical severity of cognitive decline in the face of AD pathology. Pharmacological amelioration of mitochondrial function, number, and movement deficits early in the development of AD could be a promising strategy for prevention and treatment of cognitive impairment. The current study provides evidence that $\sigma_{1} \mathrm{R}$ agonists could be useful agents to restore mitochondrial movement and number in $\mathrm{AD}$ neurons in addition to their known neuroprotective effects. More preclinical studies are needed to elucidate the impact of early mitochondrial deficits in $\mathrm{AD}$ and to develop novel treatment strategies to counteract these deficits.

\section{ACKNOWLEGEMENTS}

This work was supported by the NIH grants P20 GM109098, P01 AG027956, T32 AG052375, and U54 GM104942. Imaging experiments and image analysis were performed in the West Virginia University Microscope Imaging Facility, which has been supported by the WVU Cancer Institute and NIH grants P20RR016440, P30RR032138/P30GM103488, and P20GM103434. We thank Deborah Corbin for help with animal management, Cathy Tan and Sujung Jun for help with primary culture optimization, Amanda Ammer and Karen Martin for imaging advice, Eric Tucker and Skye Hickling for image analysis advice, Linda Nguyen for advice regarding sigma-1 receptor ligands, and $\mathrm{P}$. Mason McClatchey for providing the Matlab program for mitochondrial structure analysis.

\section{CONFLICT OF INTEREST}

The authors have no conflict of interest to report. 


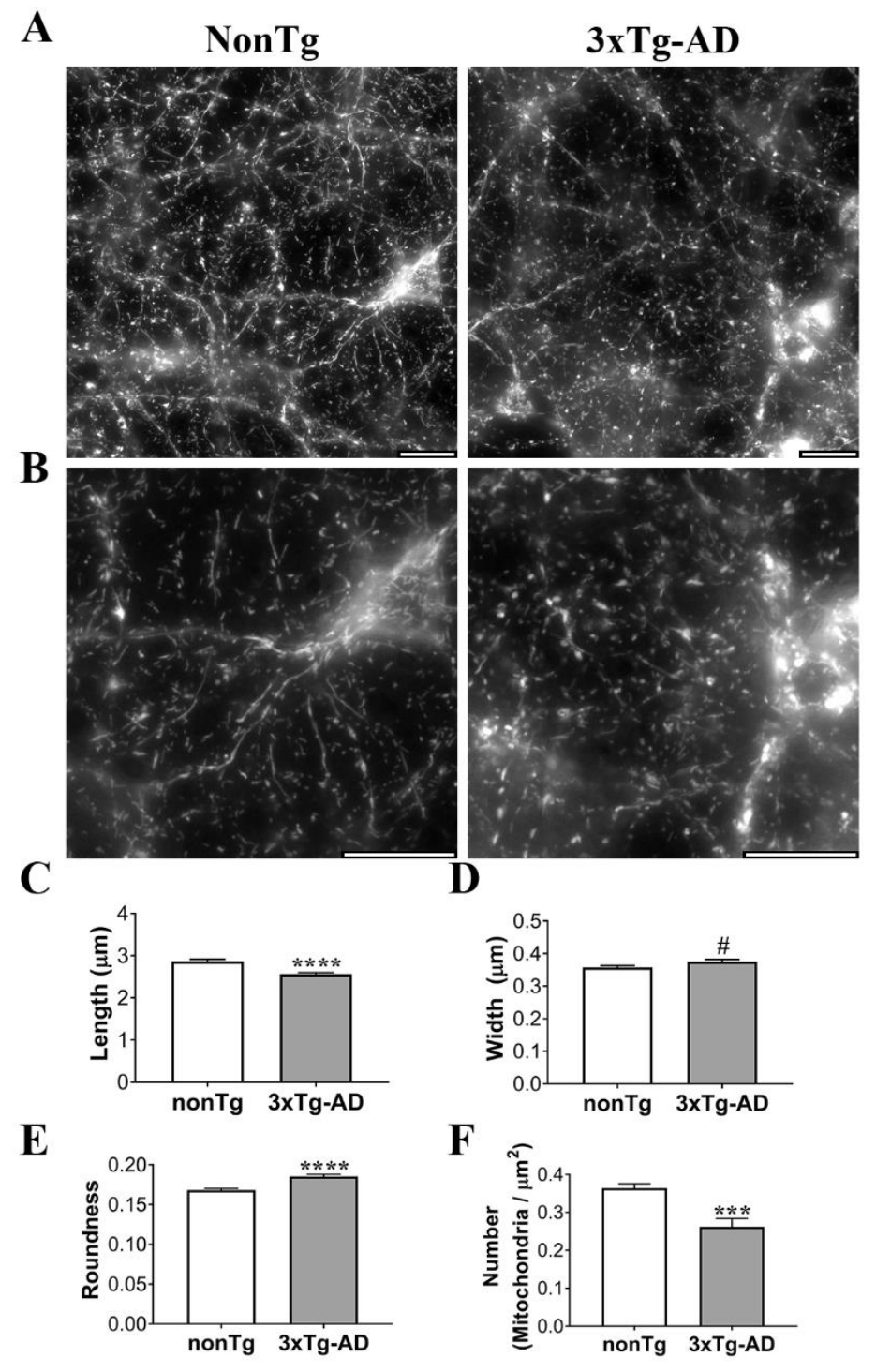

Figure 2.1. Mitochondrial structure and number in DIV12-14 primary cortical cultures.

(A) Sample fields of view from nonTg and 3xTg-AD primary cortical cultures stained with Mitotracker ${ }^{\circledR}$ Red CMXRos at 12-14 DIV. Scale bars $=20$ microns. (B) Higher magnification views of cultures. Scale bars $=20$ microns. (C) Mitochondrial length. (D) Mitochondrial width. (E) Mitochondrial roundness (width/length). (F) Mitochondrial number per square micron. $n=11$ nonTg, $\mathrm{n}=11$ 3xTg-AD cultures; bar graphs show means + SEM; \#p $<0.10 ; * * * \mathrm{p}<0.001$; $* * * * \mathrm{p}<0.0001$ 
A
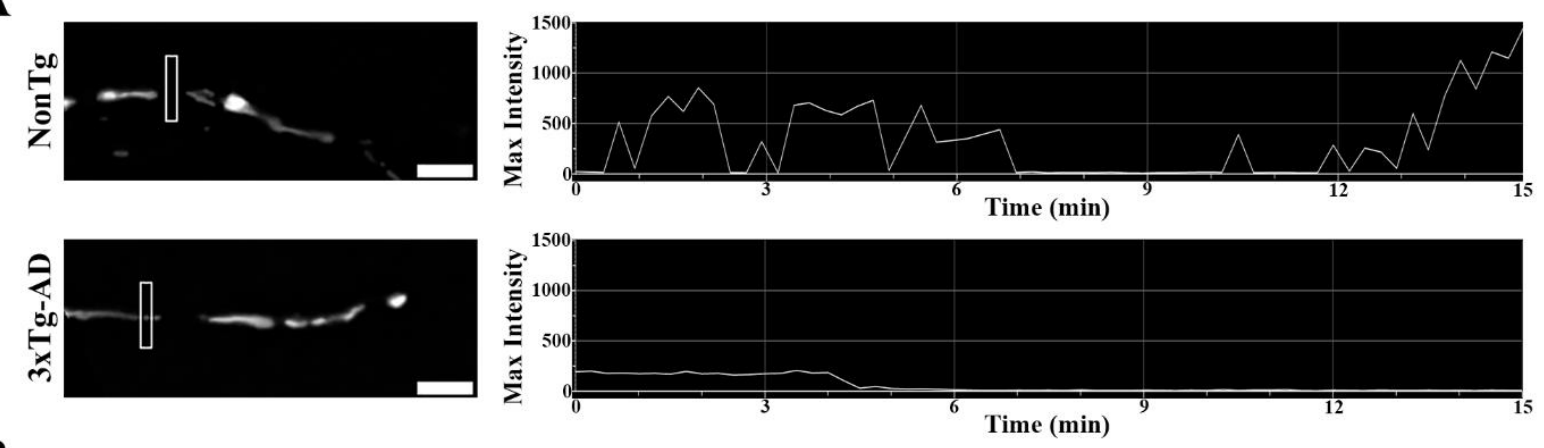

B

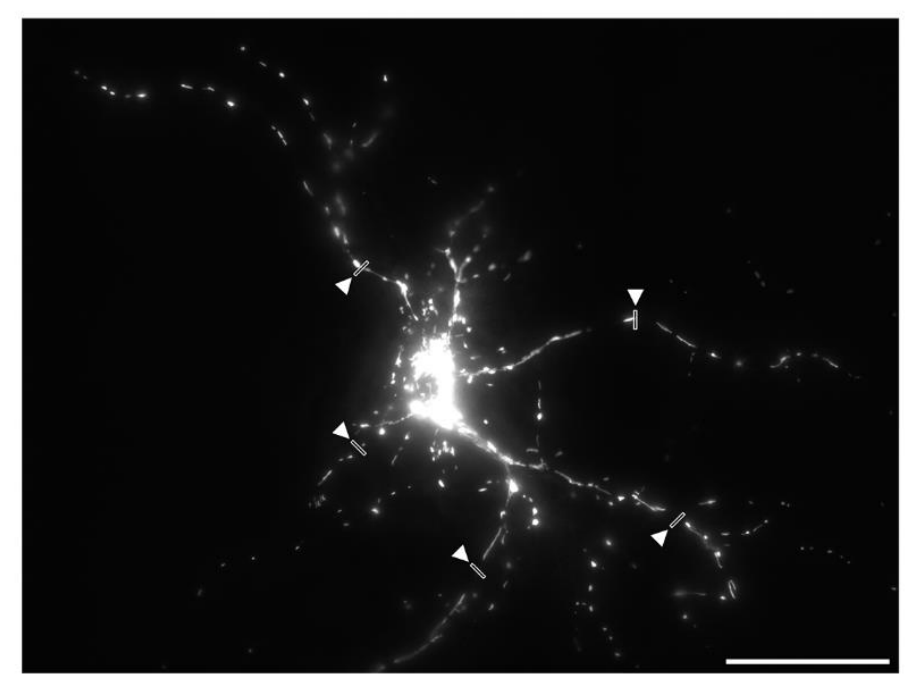

C

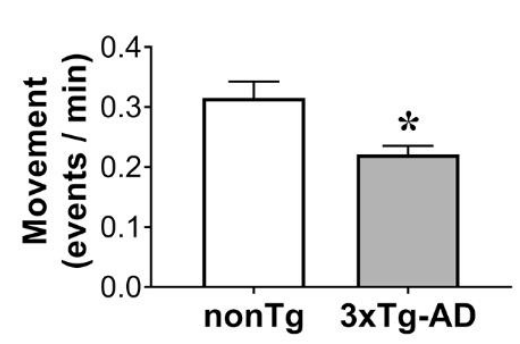

D

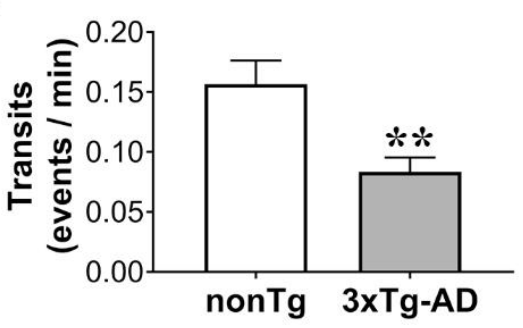

Figure 2.2. Mitochondrial movement in DIV14 primary cortical cultures. (A) Representative regions of interest within neurites of pDsRed2-mito-transfected nonTg and 3xTg-AD primary cortical neurons imaged at 14 DIV (Left). Scale bars $=5$ microns. Maximum fluorescence intensity within regions of interest over first 15 min of imaging (Right). (B) Representative nonTg neuron showing locations of regions of interest for sampling mitochondrial movement. Scale bar $=50$ microns. $(\mathrm{C})$ Number of mitochondrial movement events / minute in regions of interest. A movement event is defined by an increase or decrease in maximum fluorescence intensity of $\geq 200$ fluorescence units over $\leq 30$ seconds corresponding to movement into or out of a region of interest. (D) Number of mitochondrial transits / minute in regions of interest. A mitochondrial transit is defined as an event in which an individual mitochondrion passes 
completely through a region of interest. $\mathrm{n}=8$ nonTg, $\mathrm{n}=73 \mathrm{xTg}$-AD cultures; bar graphs show means + SEM; $* \mathrm{p}<0.05 ;{ }^{* *} \mathrm{p}<0.01$. 


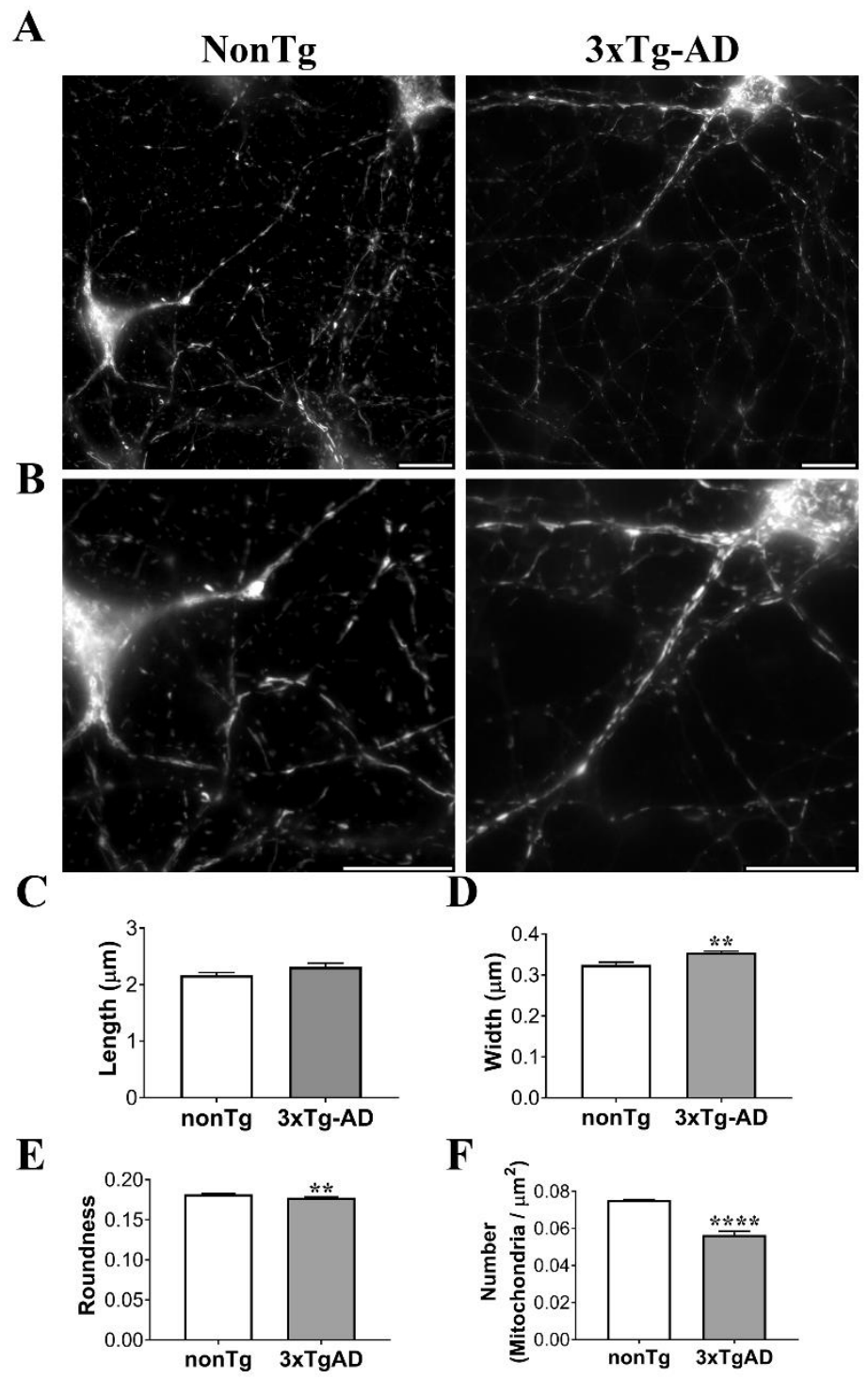

Figure 2.3. Mitochondrial structure and number in DIV7-9 primary cortical cultures. (A)

Sample fields of view from nonTg and 3xTg-AD primary cortical cultures stained with Mitotracker® Red CMXRos at 7-9 DIV. Scale bars = 20 microns. (B) Higher magnification views of cultures. Scale bars $=20$ microns. (C) Mitochondrial length. (D) Mitochondrial width. (E) Mitochondrial roundness (width/length). (F) Mitochondrial number per square micron. $\mathrm{n}=6$ nonTg, $\mathrm{n}=6$ 3xTg-AD cultures; bar graphs show means $+\mathrm{SEM} ; * * \mathrm{p}<0.01 ; * * * * \mathrm{p}<0.0001$. 


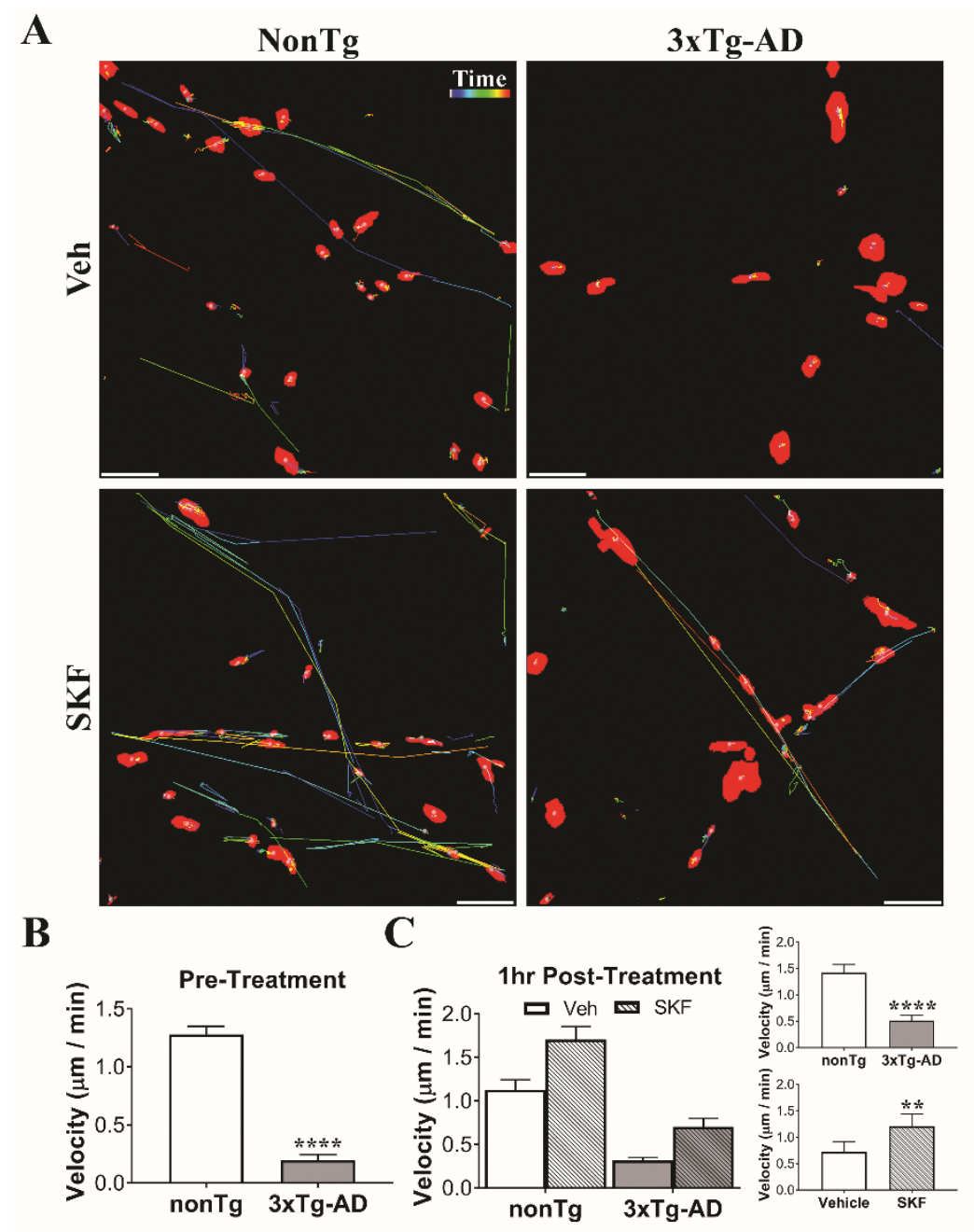

Figure 2.4. Mitochondrial movement in DIV7-9 primary cortical cultures. (A) Movement traces of nonTg and 3xTg-AD neurons one hour post-vehicle (Veh) or $1 \mu \mathrm{mol} / \mathrm{L}(+) \mathrm{SKF}-10,047$ (SKF) treatment. Scale bars $=4$ microns. (B) Pre-treatment mitochondrial velocity of nonTg and 3xTg-AD cultures. $n=6$ nonTg, $n=63 x T g-A D$ cultures. $(C)$ (Left) Mitochondrial velocity in nonTg and 3xTg-AD cultures one hour post-treatment with vehicle (Veh) or $1 \mu \mathrm{mol} / \mathrm{L}(+) \mathrm{SKF}-$ 10,047 (SKF). Insets (Right) show main effects of genotype (Top) and treatment (Bottom). n=3 cultures per group; bar graphs show means + SEM; **p<0.01; ****p<0.0001. 
A
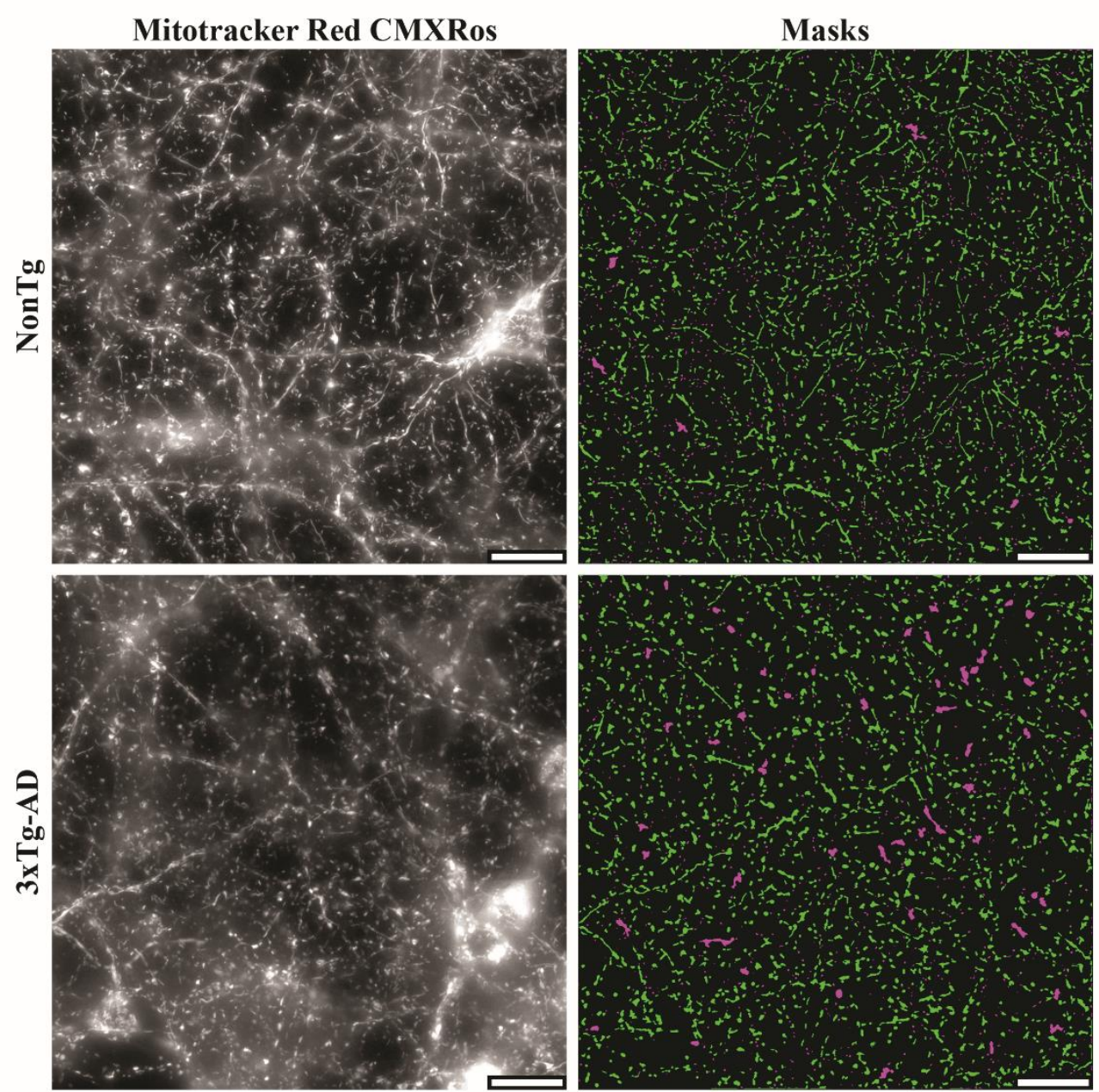

B

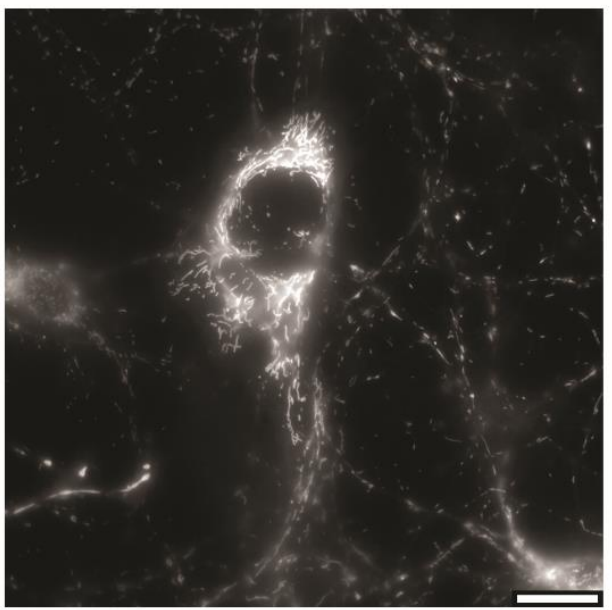

Supplemental Figure 2.1. Measurement of mitochondrial structure in primary cortical

cultures. (A) Raw images (Left) of cultures and corresponding binary masks (Right) of 
mitochondria (Green) segmented using a Matlab structural analysis program. Small or large bodies (Pink) that were excluded from analysis. (B) Field of view containing putative glial cell as defined by lack of extended neurites and presence of reticular perinuclear mitochondrial network. Such fields of view were excluded from analysis. Scale bars $=20$ microns. 
A
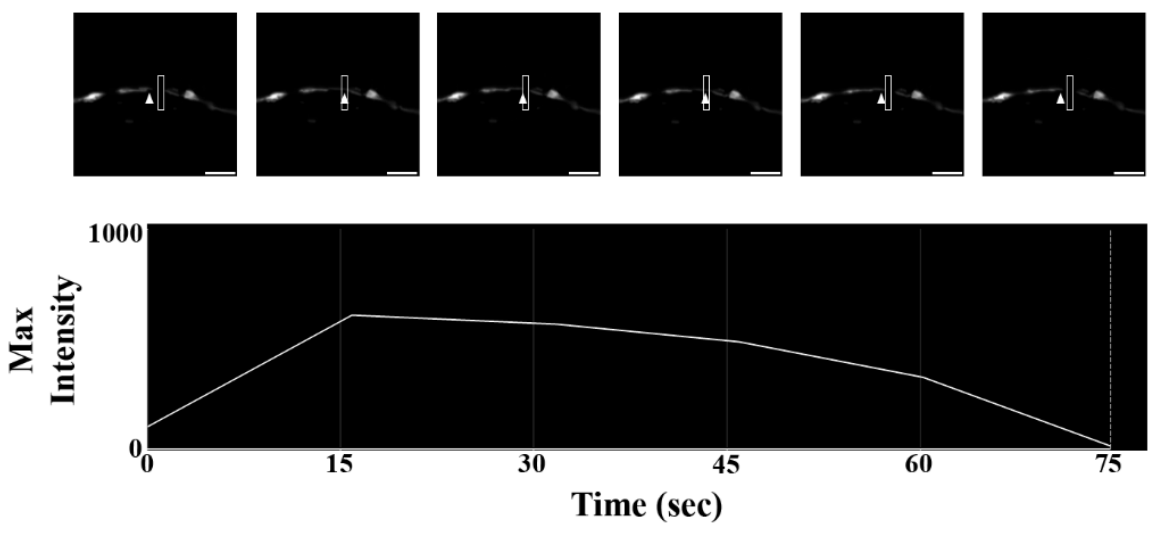

B
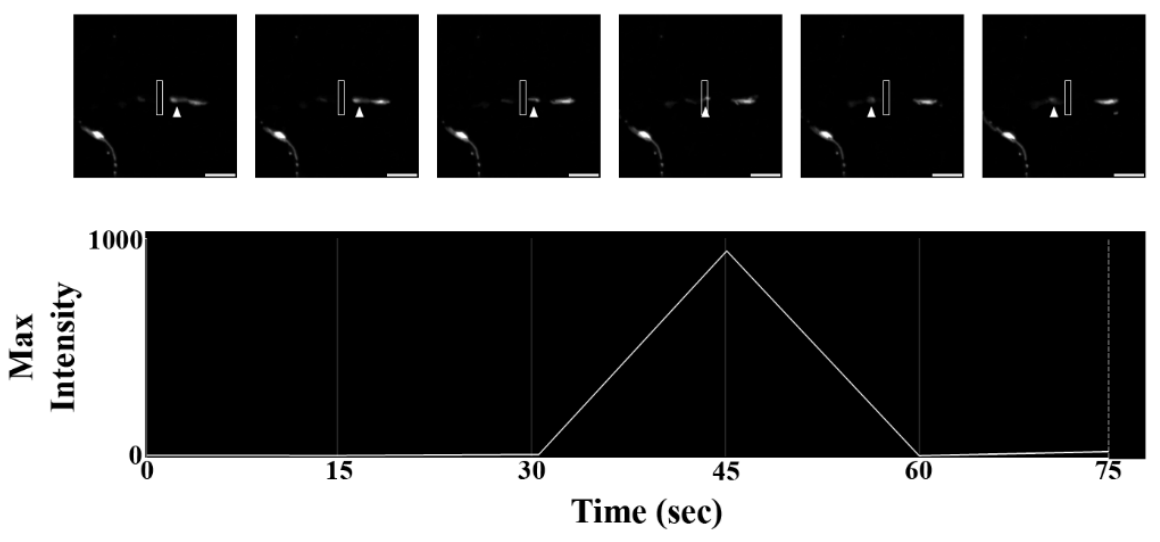

Supplemental Figure 2.2. Mitochondrial movement and mitochondrial transit events. (A)

Example of two mitochondrial movement events as defined by an increase or decrease in maximum fluorescence intensity of $\geq 200$ fluorescence units over $\leq 30$ seconds. One event is represented by movement into and one by movement out of the region of interest. (B) Example of a mitochondrial transit, as defined by a mitochondrial movement event in which an individual mitochondrion passes completely through a region of interest. Regions of interest $=1.1$ microns x 6 microns; Scale bars $=5$ microns. 


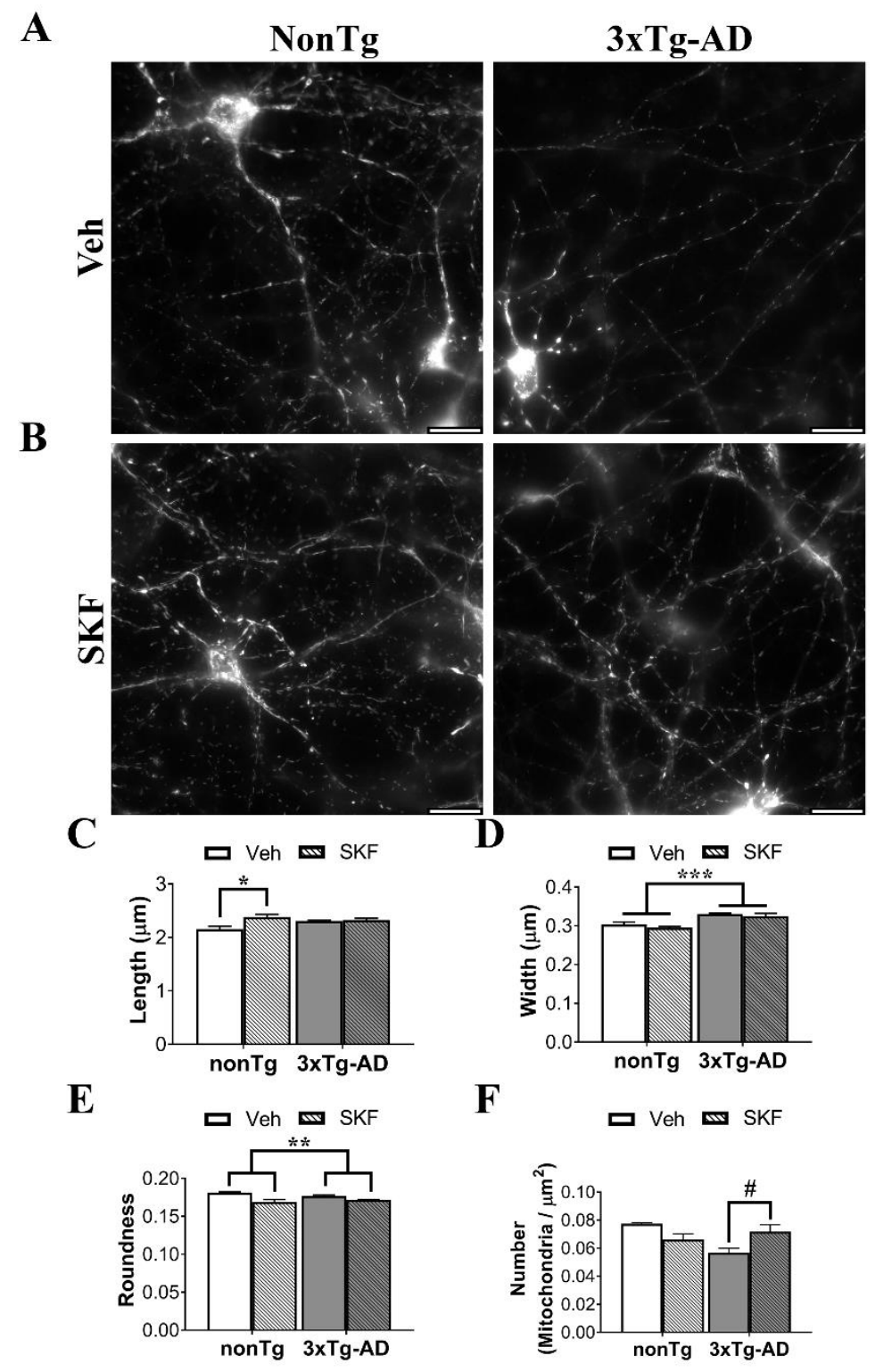

Supplemental Figure 2.3. Effect of (+)SKF-10,047 on mitochondrial structure and number in DIV7-9 primary cortical cultures. (A) Sample fields of view from nonTg and 3xTg-AD primary cortical cultures stained with Mitotracker® Red CMXRos, imaged one hour post-vehicle (Veh) or $1 \mu \mathrm{mol} / \mathrm{L}(+)$ SKF-10,047 (SKF) treatment. (B) Average mitochondrial length. (C) Average mitochondrial width. (D) Average mitochondrial roundness (width/length). (E) Average 
mitochondrial number per square micron. $n=3$ cultures per group; bar graphs show means + $\mathrm{SEM} ; \# \mathrm{p}<0.10 ;{ }^{*} \mathrm{p}<0.05 ; * * \mathrm{p}<0.01 ; * * * \mathrm{p}<0.001$ 


\section{Chapter 3}

\section{Mitochondrial Structure and Number in Cortical and Hippocampal Neurons of Aged 3xTg-AD Mice}

John Z. Cavendish, Saumyendra N. Sarkar, Mark A. Colantonio, Dominic D. Quintana, Sujung Jun, and James W. Simpkins 


\begin{abstract}
:
Mitochondrial dysfunction is observed early in the development and consistently throughout the course of Alzheimer's disease (AD). Mitochondrial number and structural abnormalities have been observed in human $\mathrm{AD}$ patients and $\mathrm{AD}$ mouse models in cortical and hippocampal neurons in areas related to AD pathology. Previous studies of mitochondrial morphology in AD have utilized mostly electron microscopy, which has the advantage of providing ultrastructural detail but lacks the ability to examine large numbers of mitochondria across a wide range of brain areas. In the current study, we crossed mice expressing mitochondrially targeted cyan fluorescent protein (mito-CFP) with triple-transgenic Alzheimer's disease (3xTg-AD) mice through successive breeding of offspring to create homozygous 3xTg-AD-CFP mice and nonTg-CFP controls. We used confocal microscopy of coronal brain sections to study mitochondrial number, volume, and sphericity in the primary motor cortex (M1), primary somatosensory cortex (S1), anterior cingulate cortex (AC), entorhinal cortex (EC), and CA1 region of the hippocampus in 24-month mice at the latest stages of AD pathology. We also studied mitochondrial structure and number in anatomically-defined subregions of the CA1 and dentate gyrus areas of the hippocampus. We found structural differences in the AC and CA1, particularly in the stratum radiatum layer. We did not observe any difference in mitochondrial number between AD and nonTg mice in any of the areas examined. These results provide evidence that metabolic deficiencies in $\mathrm{AD}$ are likely the result of declining mitochondrial function, not number, and that mitochondrially targeted treatments may be able to alleviate AD symptoms even late in disease progression.
\end{abstract}




\section{Introduction:}

Mitochondrial dysfunction is a known pathological hallmark of Alzheimer's disease (AD). AD patients show reductions in cerebral glucose metabolism in areas associated with AD pathology including hippocampus, parietotemporal cortex, and cingulate cortex years before the onset of AD symptoms [3]. Homologous regional alterations in brain metabolism are seen early in life in the triple-transgenic Alzheimer's disease (3xTg-AD) mouse model of AD, and by 18 months all brain areas show reduced glucose metabolism [67]. Human AD patients also show alterations in TCA cycle enzymes in the brain including reduced activity of pyruvate dehydrogenase, isocitrate dehydrogenase, and alpha-ketoglutarate dehydrogenase and increased activity of succinate dehydrogenase and malate dehydrogenase $[68,69]$. These alterations correlate strongly with the clinical stage of disease. The 3xTg-AD mouse similarly shows reduced pyruvate dehydrogenase protein levels and mitochondrial respiration in the brain as early as 3 months of age and reduction in mitochondrial respiration of embryonic hippocampal neurons [70].

In addition to alterations of levels and activity of mitochondrial enzymes, many other mechanisms may underlie AD-associated metabolic deficits. Studying mitochondrial structure, number, and distribution in $\mathrm{AD}$ neurons is a robust way to provide more insight into multiple mechanistic pathways, including reduced mitochondrial biogenesis, alteration of fission-fusion dynamics, and impairment of mitochondrial translocation to distal cellular compartments. Two studies of $\mathrm{AD}$ patients showed reduction in number of neuronal mitochondria $[14,76]$. Changes to neuronal mitochondrial structure, including reduced mitochondrial size, were seen in the hippocampus, auditory cortex, and frontal cortex, as well as several other non-AD associated brain areas of AD patients [76]. Abnormal mitochondrial structure, including paracrystalline 
inclusions between mitochondrial cristae and increased matrix density, was also seen in dendrites of neurons in the frontal cortex of AD patients [77]. Interestingly, the presence of abnormal mitochondria in this study coincided with cortical areas with more severe dendritic degeneration. One study described a unique mitochondrial structural phenotype resembling "mitochondria on a string" in the hippocampus and entorhinal cortex of human AD patients as well as in the hippocampus of various strains of transgenic AD mice [78]. An in vivo study of mitochondrial structure in APP/PS1 mice showed reduced mitochondrial density and abnormal mitochondrial structure in the immediate vicinity of amyloid plaques [79].

A limitation of previous studies of mitochondrial structure and function in $\mathrm{AD}$ is the relatively narrow selection of mitochondria imaged in limited brain regions. Electron microscopy samples and in vivo imaging fields contain numbers of mitochondria on the order of hundreds, which represent only a small portion of total mitochondria. The purpose of our current study was to examine mitochondrial structure, number, and distribution in neurons of various brain areas in a more widespread and inclusive manner. To achieve this, we created crosses of 3xTg-AD mice [23] with mice expressing cyan fluorescent protein (CFP) targeted to a subunit of cytochrome c oxidase and under control of the Thy1 promotor [42]. Using these 3xTg-AD-CFP mice and nontransgenic-CFP (nonTg-CFP) mice derived from the same lineage, we were able to measure structure and number in large samples of mitochondria in various brain areas and identify alterations in 3xTg-AD mice at 24 months during the most severe stage of disease. We chose to examine the CA1 region of hippocampus, entorhinal cortex (EC), and anterior cingulate cortex (AC) as areas associated with a high amount of AD pathology. We chose the primary motor cortex (M1) and primary somatosensory cortex (S1) as areas not traditionally affected as heavily 
by AD pathology. We also carried out a more detailed analysis of mitochondrial structure and number in anatomically defined layers of the CA1 and dentate gyrus regions of the hippocampus.

Based on previous studies, we hypothesized that $3 \times \mathrm{Tg}-\mathrm{AD}$ mice would show reduced number and volume of mitochondria and increased mitochondrial sphericity in neurons of CA1, $\mathrm{EC}$, and $\mathrm{AC}$, with no difference in number or structure in M1 and S1. We also hypothesized that mitochondrial structural and number alterations in 3xTg-AD mice would be more severe in the stratum radiatum of the CA1 where distal pyramidal dendrites exist compared to the pyramidal layer where pyramidal cell bodies exist due to reduced capacity for AD mitochondria to travel from their site of biogenesis in the cell body to distal dendrites. We hypothesized there would be fewer alterations in mitochondrial structure and number in the dentate gyrus regions due to relatively lower burden of $\mathrm{AD}$ pathology compared to $\mathrm{CA} 1$.

\section{Materials and Methods:}

Mice

Animal protocols were performed in accordance with the National Institutes of Health Guide for the Care and Use of Laboratory Animals and were approved by the Institutional Animal Care and Use Committee of West Virginia University. Homozygous 3xTg-AD mice

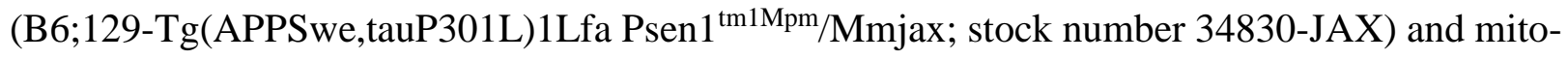
CFP mice (B6.Cg-Tg(Thy1-CFP/COX8A)S2Lich/J; stock number 007967) [42] were obtained from The Jackson Laboratory, and colonies were bred and maintained at West Virginia University. Animals were housed in cages of up to five animals on a $12 \mathrm{~h}$ light cycle with ad libitum access to food and water. 
To obtain animals used in the study, homozygous female 3xTg-AD mice were crossed with homozygous male Mito-CFP mice to obtain F1 heterozygotes. F1 heterozygotes were bred to obtain F2 mice of various genotypes which were selectively bred to obtain F3 homozygous 3xTg-AD Mito-CFP (AD-CFP) mice and nonTg Mito-CFP (nonTg-CFP) mice from which additional F4 homozygotes were bred. We used the following genotypes in F2 to breed the desired F3 AD-CFP homozygotes: (1) $\mathrm{CFP}^{+/+}, \mathrm{Ps}^{+/+}, \mathrm{APP} / \mathrm{Tau}^{+/-}$; (2) $\mathrm{CFP}^{+/+}, \mathrm{Ps}^{+/-}, \mathrm{APP} / \mathrm{Tau}^{+/+}$; (3) $\mathrm{CFP}^{+/+}, \mathrm{Ps}^{+/-}, \mathrm{APP} / \mathrm{Tau}^{+/-}$. We used the following genotypes in F2 to breed the desired F3

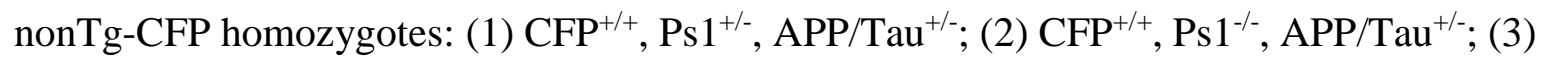
$\mathrm{CFP}^{+/+}, \mathrm{Ps}^{+/-}, \mathrm{APP} / \mathrm{Tau}^{-/-}$. Therefore, homozygous nonTg-CFP and AD-CFP mice were both derived as F3 progeny of the same founder mice, and all mice used in this study were either the F3 mice or their F4 offspring from crosses between $\mathrm{F} 3 \mathrm{CFP}^{+/+}, \mathrm{Ps}^{+/+}$, $\mathrm{APP} / \mathrm{Tau}^{+/+}$parents for $\mathrm{AD}-\mathrm{CFP}$ mice or $\mathrm{F} 3 \mathrm{CFP}^{+/+}, \mathrm{Ps}^{-/-}, \mathrm{APP} / \mathrm{Tau}^{-/-}$parents for nonTg-CFP mice.

\section{Solutions}

10x Phosphate buffered saline (PBS) was prepared by dissolving $80 \mathrm{~g} \mathrm{NaCl}, 2 \mathrm{~g} \mathrm{KCL}$, 11.5 $\mathrm{g} \mathrm{Na}_{2} \mathrm{HPO}_{4} \cdot 7 \mathrm{H}_{2} \mathrm{O}$, and $2 \mathrm{~g} \mathrm{KH}_{2} \mathrm{PO}_{4}$ in milliQ water, bringing volume to $1 \mathrm{~L}$, and adjusting $\mathrm{pH}$ to 7.5. 1x PBS was made by diluting 10x PBS 1:10 in milliQ water. For 20\% paraformaldehyde (PFA), 80g PFA was dissolved in warm milliQ water and volume was brought to $400 \mathrm{~mL} .4 \%$ PFA was prepared by mixing 350mL milliQ water, 100mL 20\% PFA, and 50mL 10x PBS and adjusting $\mathrm{pH}$ to 7.4 . $30 \%$ sucrose solution was prepared by dissolving $30 \mathrm{~g}$ sucrose in $1 \mathrm{x}$ PBS and adjusting volume to $100 \mathrm{~mL}$.

\section{Tissue Preparation}


24-month mice were perfused transcardially with 20-30 mL of 1x PBS to remove blood followed by $25 \mathrm{~mL} 4 \%$ PFA solution. Brains were dissected out and post-fixed for 24 hours in 4\% PFA. Brains were then washed with 1x PBS and transferred to $30 \%$ sucrose solution for 72 hours for cryoprotection. Following cryoprotection, brains were stored at $4^{\circ} \mathrm{C}$ in $30 \%$ sucrose until slicing. Prior to slicing, olfactory bulbs and cerebellum were removed. Brains were mounted on a sliding microtome with freezing stage (Thermo) and allowed to freeze fully. $50 \mu \mathrm{m}$ coronal slices were made through the entire cortex and collected in 1x PBS. Slices were mounted onto glass slides in mounting medium (Ibidi) containing 1,4-diazabicyclo[2.2.2]octane (DABCO) and covered with glass coverslips (0.17 mm thickness). Slides were stored in the dark at $4^{\circ} \mathrm{C}$ until imaging.

\section{Imaging}

Three representative slices per area containing AC, M1, S1, and entorhinal cortex as well as CA1 hippocampus were chosen for each animal for imaging (Fig. 3.1D). The slices imaged for each animal represented the same antero-posterior position in the brain for each area. Areas were confirmed using the Allen Adult Mouse Brain Atlas. Confocal images were collected using a Zeiss 710 laser scanning microscope with a 63x Plan Apochromat oil immersion objective (N.A.=1.4) at an excitation wavelength of $458 \mathrm{~nm}$, laser power of $35.1 \%$, gain of 923 , and digital offset of 44.4. A 465-518nm emission filter was used to collect fluorescence emitted from CFP. Z-stacks were collected through the entire thickness of the slice at an interval of $0.39 \mu \mathrm{m}$. $1024 \times 1024$ resolution images were acquired with xy pixel scale of $0.132 \mu \mathrm{m} /$ pixel to give an imaging field of view of $135 \times 135 \mu \mathrm{m}^{2}$. For comparisons of 24-month AD-CFP vs. nonTg-CFP mice, a single field of view was captured for each slice giving a total of 3 fields of view for each imaging area. For subregion analysis of the hippocampus, 10x3 stitched z-stacks were captured 
encompassing a central strip of CA1 and dentate gyrus. The hippocampal region imaged for subregion analysis was on the opposite side of the slice as the area used for single field of view imaging so that no part of CA1 was sampled more than once.

\section{Data Analysis}

\section{$\underline{\text { Single Field of View Images }}$}

Zeiss images were converted to stacks of 8-bit Tiffs and imported into ImageJ (NIH). A substack of 60 images $(23.4 \mu \mathrm{m})$ was selected starting from the first plane fully encompassing the sample with no hole-artifacts at the cut surface of the tissue. Images were despeckled and background subtraction (rolling ball radius $=10$ pixels) was performed. Processed Tiff stacks were exported to Imaris (Bitplane) for 3D rendering. The surface function was used to segment mitochondria in 3D-rendered images with auto-thresholding enabled. A filter was used to exclude objects with volume $\leq 0.10 \mu \mathrm{m}^{3}$ which represent noise. The volume and sphericity of each object were measured as well as the total number of objects present. Average object volume and sphericity for each imaging area were calculated by pooling the measurements of objects from the 3 slices imaged and taking the grand average. Average number of objects was calculated by averaging the three measurements of number of objects for each area.

\section{$\underline{\text { Subregions of CA1 and Dentate Gyrus }}$}

Stitched images were cropped into regions representing five subregions (Stratum OriensSO, Pyramidal Layer-Pyr, Superficial Stratum Radiatum-SRs, Deep Stratum Radiatum-SRd, Stratum Lacunosum Moleculare-SLM) for CA1 (Fig. 3.2) and three subregions (Molecular Layer-Mol, Granular Layer-Gr, and Subgranular Layer-SG) for dentate gyrus (Fig. 3.4). Each rectangle represented the same imaging volume of one field of view used in the single-field-of- 
view analysis. Cropped zeiss images were converted to stacks of 8-bit Tiffs and exported to ImageJ (NIH). A substack of 60 images $(23.4 \mu \mathrm{m})$ was selected starting from the first plane fully encompassing the sample with no hole-artifacts at the cut surface of the tissue. Images were despeckled and background subtraction (rolling ball radius $=10$ pixels) was performed. Processed Tiff stacks were exported to Imaris (Bitplane) for 3D rendering. The surface function was used to segment mitochondria in 3D-rendered images with auto-thresholding enabled. A filter was used to exclude objects with volume $\leq 0.10 \mu \mathrm{m}^{3}$ which represent noise. The volume and sphericity of each object were measured as well as the total number of objects present. Average object volume and sphericity for each imaging area were calculated by pooling the measurements of objects from the 3 slices imaged and taking the grand average. Average number of objects was calculated by averaging the three measurements of number of objects for each area.

\section{$\underline{\text { Statistical Analysis }}$}

Investigators performing imaging and image analysis were blinded to age and genotype. All statistical analyses were performed using Prism version 7.04 (GraphPad). Two-tailed unpaired t-tests were used to assess the effect of genotype on mean mitochondrial volume, sphericity, and number per imaging field with a significance level of $p<0.05$. For single field-ofview analyses, $n=5$ 24-month nonTg-CFP mice and n=4 24-month 3xTg-AD-CFP mice. For subregion analyses, $n=3$ 24-month nonTg-CFP mice and n=3 24-month 3xTg-AD-CFP mice. 


\section{Results:}

Mitochondrial number, volume, and sphericity in neurons of 24-month 3xTgAD and nonTg mice.

There was no difference between 24-month 3xTg-AD and nonTg mice in number of mitochondria per imaging volume in any of the four cortical or one hippocampal region imaged (Fig. 3.1A). Across all areas, 3xTg-AD mice trended towards more mitochondria, but also had more variability in mitochondrial number than nonTg mice. Mitochondrial volume was significantly higher in the AC cortex of $3 \mathrm{xTg}-\mathrm{AD}$ mice by about $33 \%(\mathrm{t}(7)=2.869, \mathrm{p}=0.024)$ and significantly lower in the CA1 hippocampus of 3xTg-AD mice by about $20 \%(\mathrm{t}(7)=2.024$, $\mathrm{p}=0.041)$ (Fig. 3.1B). Conversely, mitochondrial sphericity was significantly lower in neurons of $\mathrm{AC}$ cortex $(\mathrm{t}(7)=5.738, \mathrm{p}=0.0007)$ and higher in neurons of CA1 hippocampus $(\mathrm{t}(7)=$ $2.424, \mathrm{p}=0.046$ ) of $3 \times \mathrm{Tg}-\mathrm{AD}$ mice (Fig. 3.1C). There were no differences in mitochondrial volume or sphericity in M1, S1, or EC. Mitochondria of the CA1 hippocampus in both genotypes tended to be smaller, more numerous, and more spherical than mitochondria of the four cortical areas examined.

Mitochondrial number, volume, and sphericity in hippocampal subregions of 24-month 3xTgAD and nonTg mice

There was no significant difference between 3xTg-AD and nonTg mice in mitochondrial number in any subregion of CA1 (Fig. 3.2; Fig. 3.3A) or dentate gyrus (Fig. 3.4; Fig. 3.5A). Mitochondrial volume was significantly reduced in 3xTg-AD mice in neurons of both SRs (t(4) $=2.475, \mathrm{p}=0.034)$ and $\mathrm{SRd}(\mathrm{t}(4)=4.852, \mathrm{p}=0.008)$, but there were no differences in SO, Pyr, or SLM (Fig. 3.3C). The only CA1 area with a significant difference in mitochondrial sphericity was $\mathrm{SO}$, where $3 \mathrm{xTg}-\mathrm{AD}$ mice had significantly more spherical mitochondria $(\mathrm{t}(4)=3.474, \mathrm{p}=$ 
0.026) (Fig. 3.3B). Mitochondria of the SLM had markedly higher volume and were less numerous than any of the other four areas of CA1 in both genotypes (Fig. 3.3A,D). There were no differences in mitochondrial number, volume, or sphericity between 3xTg-AD and nonTg mice in any subregion of the dentate gyrus (Fig. 3.5A-C), but Gr had fewer mitochondria of higher volume than Mol or SG in both genotypes (Fig. 3.5A,B).

\section{Discussion:}

Mitochondrial structural changes in CA1 and AC were observed in neurons of 24-month 3xTg-AD mice compared to nonTg mice in the absence of difference in mitochondrial number. The reduction in mitochondrial volume and increase in mitochondrial sphericity in CA1 supports previous data showing hippocampal mitochondrial structural abnormalities in the hippocampus of $\mathrm{AD}$ patients and 3xTg-AD mice $[76,78]$. Contrary to these studies, however, was the fact that nonTg mice showed relatively small, spherical neuronal mitochondria in the dendritic compartment (SRs and SRd) in comparison to the long, rod-shaped mitochondria expected. Mitochondria in the cell body compartment (Pyr), however, exhibited the expected rod-shaped morphology. The increased mitochondrial volume and decreased sphericity in 3xTg-AD mice within AC neurons might be the consequence of mitochondrial inclusions within the matrix as described in frontal cortical dendrites of AD patients [77]. More detailed electron microscopy studies in 3xTg-AD-CFP mice could help determine if this were the case. The lack of differences in neuronal mitochondrial structure or number in 3xTg-AD mice compared to nonTg mice at 24months in M1 and S1 was expected, but was unexpected in EC as this is one of the first and most severely affected regions in AD. It is possible that the lack of observed mitochondrial pathology could be due to bias of imaging only the neurons which survived the AD pathology up to 24 months of age, and that neurons with more severe mitochondrial pathology had already died. 
Even if this were the case for a small population of neurons, it is unlikely that this bias had a large effect in our study as there was no observed difference in density of neuronal cell bodies between 3xTg-AD and nonTg mice in our study. Further, significant hippocampal or cortical atrophy and/or neuron loss are not reported in 3xTg-AD mice despite development of $\mathrm{AD}$ pathology [122].

There are several possible confounding factors in the interpretation of the results of this study. One is that it is unknown whether expression of mito-CFP alters mitochondrial structure or number. Few antibodies exist for immunohistochemical staining of mitochondria in fixed tissue samples, and none of them produce a clear enough signal for detailed structural analysis of individual mitochondria via light microscopy. Therefore it was not possible to compare nonCFP-expressing mice with CFP-expressing mice using the microscopy methods from this study. An alternative option would be to perform electron microscopy analysis of mitochondrial structure and number in non-CFP-expressing mice compared to CFP-expressing mice, but the sampling region would be necessarily limited.

Another possible limitation to this study was that mitochondrial structure may be altered during the perfusion and fixation process. There is a necessary, yet brief exposure of neurons to hypoxia during desanguination and before fixation, which may induce a structural response in mitochondria such as that seen in primary astrocytes [123]. Also, fixation at room temperature, as was performed in this study, has been shown to reduce mitochondrial length in cultured cells compared to fixation at $37^{\circ} \mathrm{C}[110]$. It is possible to image mitochondria in the cortex of live, anesthetized mito-CFP mice through a cranial window [124], so future studies might be able to elucidate the differences in mitochondrial structure and number in live neurons versus fixed tissue to avoid these issues. 
Overall, this study showed differences in mitochondrial structure in the CA1 and AC of 3xTg-AD mice at the most severe stage of disease without differences in mitochondrial number. Previous human and mouse studies which found significant mitochondrial $\backslash$ number changes in AD were based on observations of relatively few mitochondria using electron microscopy. The strength of this study was its inclusion of a vast number of neuronal mitochondria with unbiased sampling, and may indicate that previously observed mitochondrial structure and number alterations might be local phenomena in certain subpopulations of neurons. This study supports the conclusion that metabolic deficits in the AD brain are more heavily related to mitochondrial function than number. This gives promise to the prospect of using pharmacotherapy to restore mitochondrial energy production, as the requisite mitochondria are likely still present even at advanced stages of pathology. 


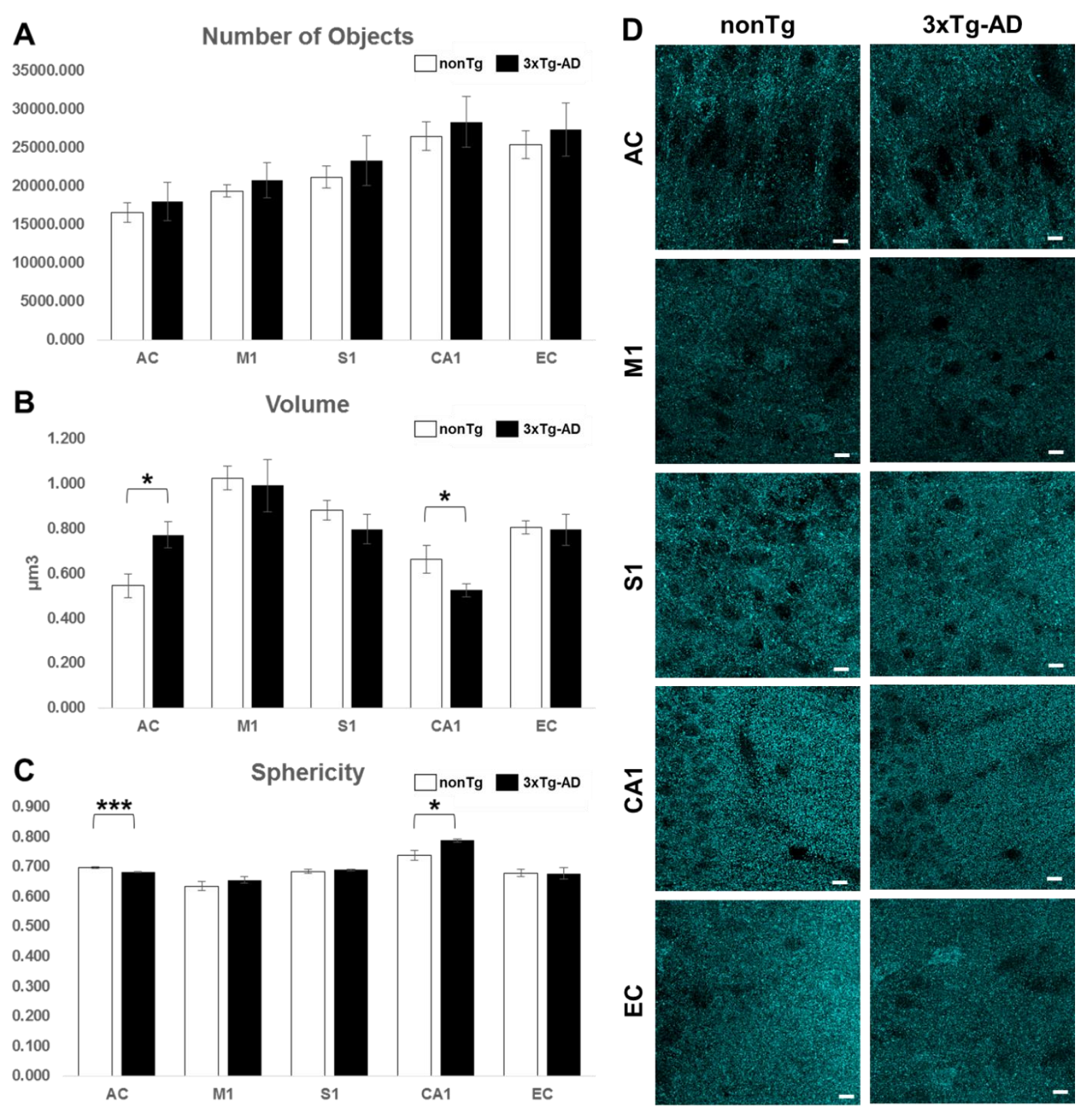

Fig. 3.1. Neuronal mitochondrial number, volume, and sphericity in 24-month 3xTgAD and nonTg mice. (A) Number of objects per imaging volume. (B) Mitochondrial volume. (C) Mitochondrial sphericity. Values are mean \pm standard error. ${ }^{*} p<0.05 ; * * * p<0.001 . \mathrm{n}=5$ (WT); $\mathrm{n}=4$ (3xTgAD). (D) Representative images of brain areas showing neuronal mitochondria (cyan). AC- Anterior Cingulate Cortex; M1- Primary Motor Cortex; S1- Primary Somatosensory Cortex; CA1- Hippocampus; EC- Entorhinal Cortex. Scale bars $=10 \mu \mathrm{m}$ 

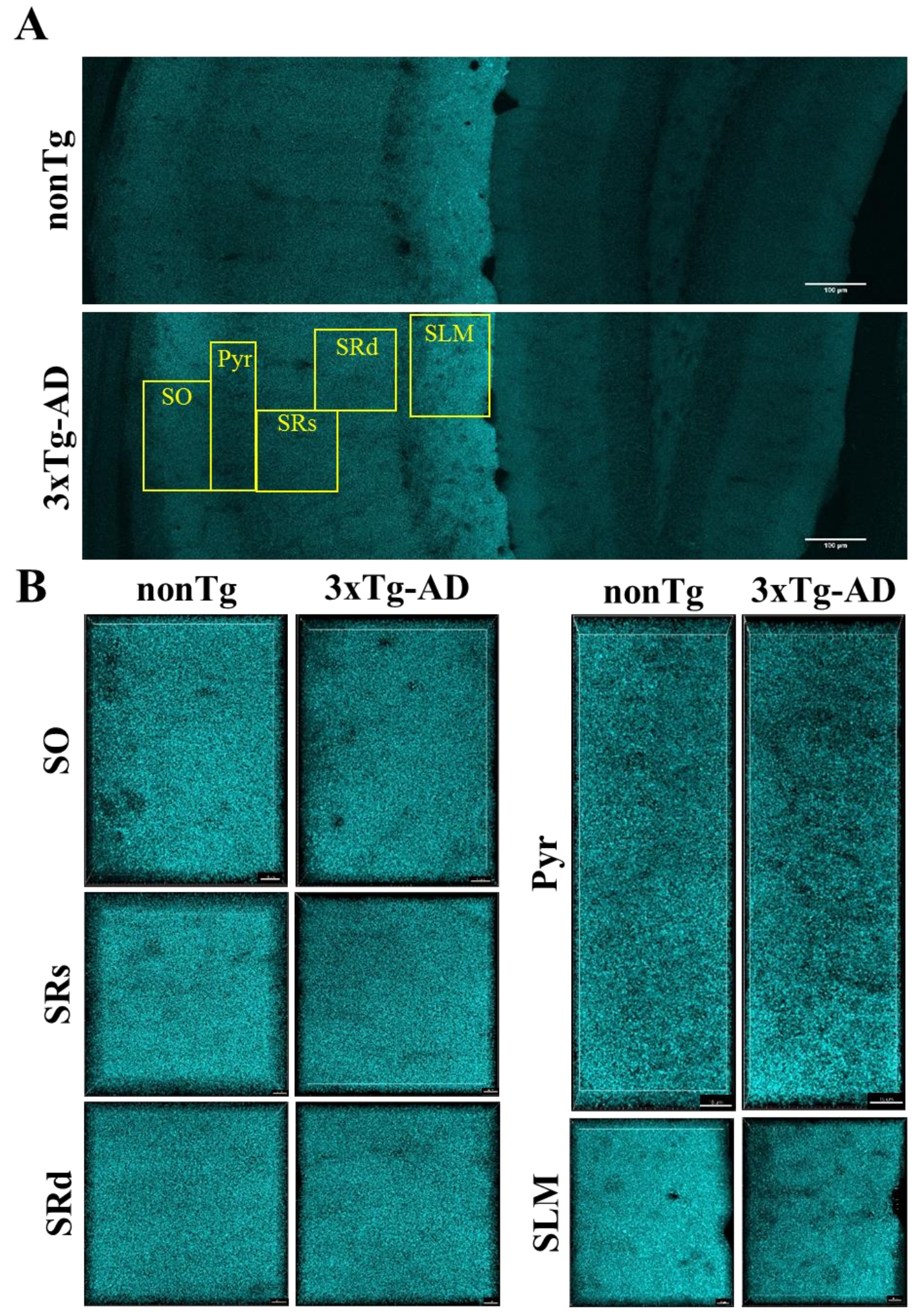

Fig. 3.2. Mitochondria in subregions of CA1 in 24-month 3xTgAD and nonTg mice. (A)

Cross-section of hippocampus showing location and size of subregions. Scale bars $=100 \mu \mathrm{m}(\mathrm{B})$ 
Subregions of CA1; SO-Stratum Oriens; Pyr-Pyramidal Layer; SRs-Superficial Stratum

Radiatum; SRd-Deep Stratum Radiatum; SLM-Stratum Lacunosum Moleculare. Scale bars = $10 \mu \mathrm{m}(\mathrm{SO}, \mathrm{SRs}, \mathrm{SRd}$, and SLM); Scale bars $=15 \mu \mathrm{m}(\mathrm{Pyr})$. 


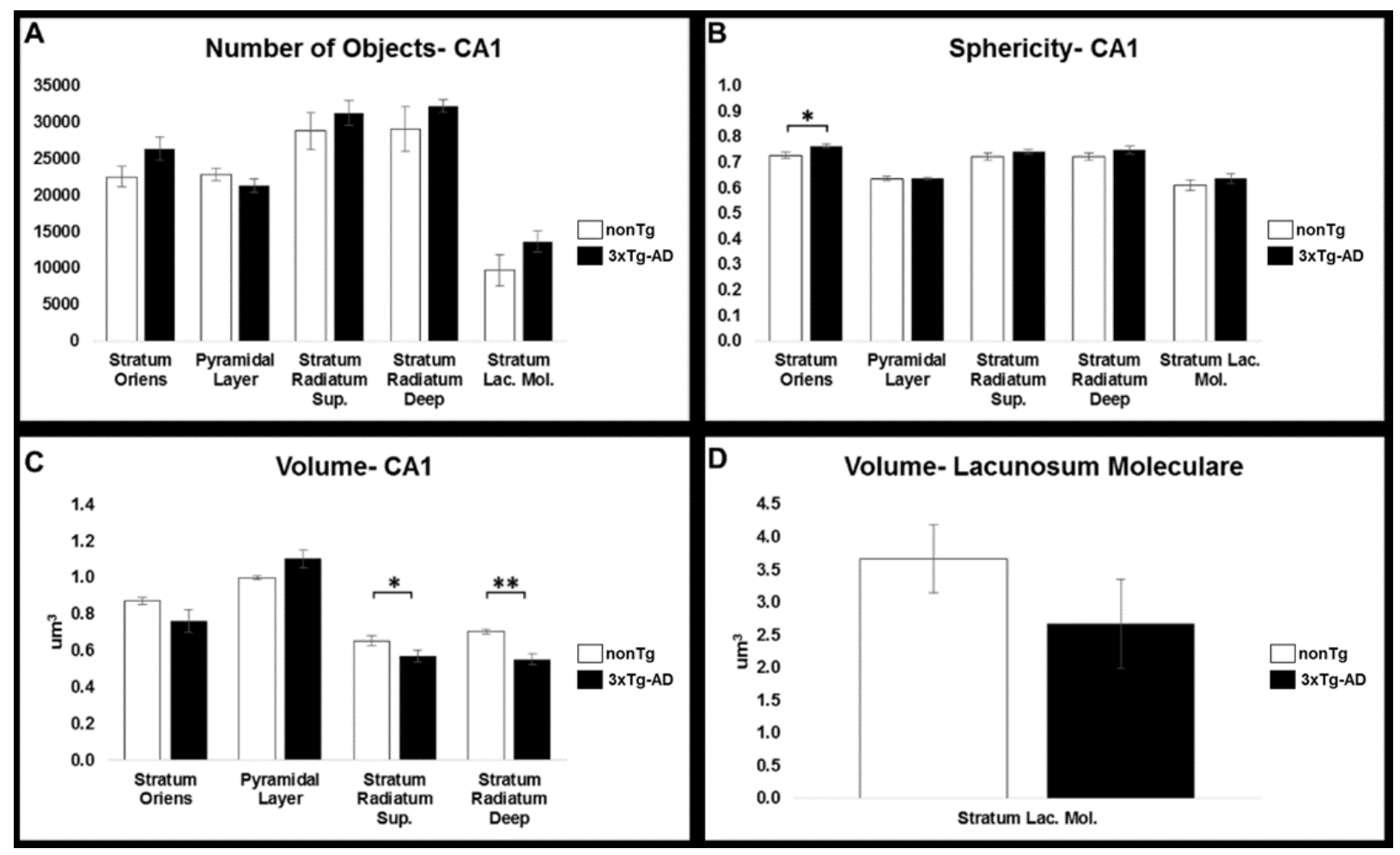

Fig. 3.3. Neuronal mitochondrial number, volume, and sphericity in CA1 Subregions of 24month 3xTgAD and nonTg mice. (A) Number of objects per imaging volume. (B)

Mitochondrial sphericity. (C-D) Mitochondrial volume. Values are mean \pm standard error. ${ }^{*} p<$ $0.05, * * p<0.01 . \mathrm{n}=5(\mathrm{WT}) ; \mathrm{n}=4(3 \times \mathrm{TgAD})$. 


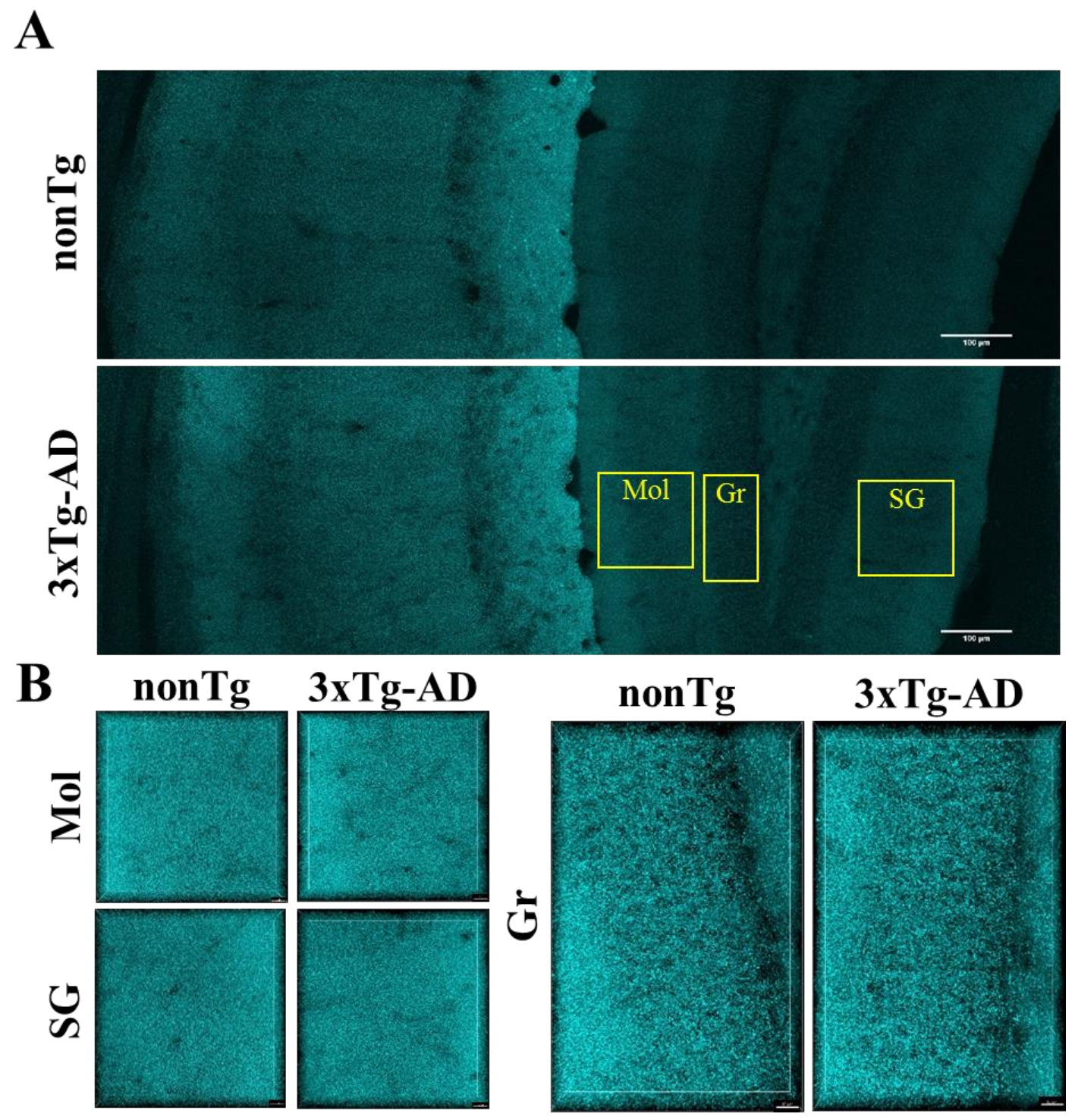

Fig. 3.4. Mitochondria in subregions of dentate gyrus in 24-month 3xTgAD and nonTg mice. (A) Cross-section of hippocampus showing location and size of subregions. Scale bars = $100 \mu \mathrm{m}$ (B) Subregions of dentate gyrus; Mol-Molecular Layer; Gr-Granular Layer; SGSubgranular Layer. Scale bars $=10 \mu \mathrm{m}$. 


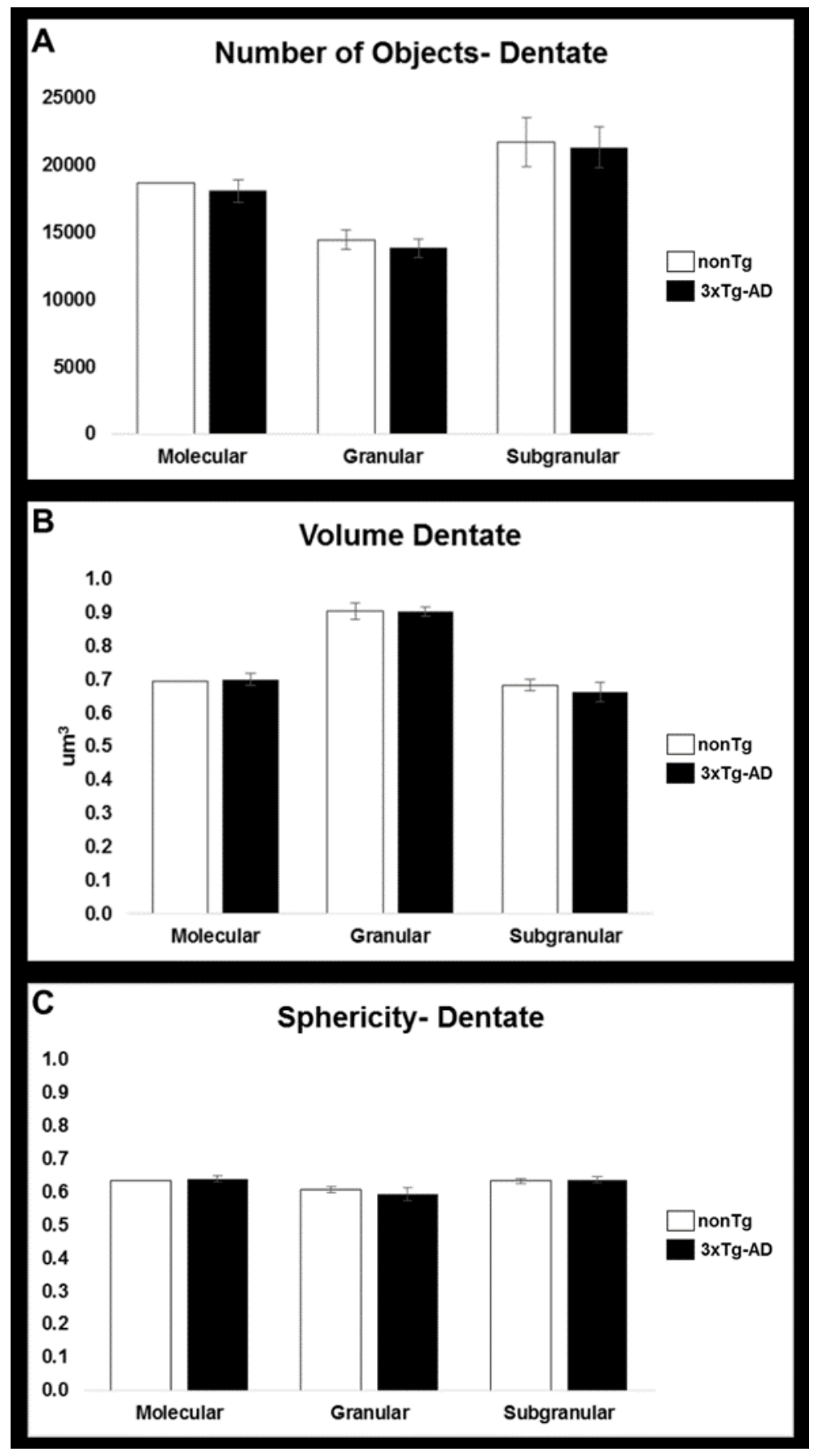

Fig. 3.5. Neuronal mitochondrial number, volume, and sphericity in dentate gyrus

subregions of 24-month 3xTgAD and nonTg mice. (A) Number of objects per imaging volume

(B) Mitochondrial volume. (C) Mitochondrial sphericity. Values are mean \pm standard error. ${ }^{*} p<$ 0.05. $\mathrm{n}=5(\mathrm{WT}) ; \mathrm{n}=4(3 \times \mathrm{TgAD})$. 


\section{Chapter 4}

\section{Discussion}




\section{Discussion}

Because of the high dependence upon mitochondrial energy production in neurons and the unique challenges posed to neuronal mitochondria, neurons are especially susceptible to degeneration following mitochondrial dysfunction. Previous work has suggested that mitochondria are abnormal in function, morphology, number, and dynamics early in and consistently throughout the course of AD. Some have hypothesized that mitochondria are critically involved in the initiation and propagation of sporadic $\mathrm{AD}$, and that declining mitochondrial function precedes and underlies the development of the other hallmarks of AD pathology including tau hyperphosphorylation and aggregation, $\mathrm{A} \beta$ overproduction, and neuronal/synaptic loss $[17,125]$. Studying the contribution of mitochondrial abnormalities to the progression of $\mathrm{AD}$ and the time-course of their appearance are important in understanding the full picture of $\mathrm{AD}$ pathology and developing new treatment strategies which can protect and/or restore mitochondrial function at critical stages of $\mathrm{AD}$ progression. The failure of amyloid-based AD treatments initiated late in disease progression underlies the need for new approaches. Targeting mitochondrial dysfunction early in AD-susceptible individuals should be explored as part of a multifactorial approach to preventing or slowing the disease. My dissertation research examined mitochondrial changes at the earliest and latest stages of disease in 3xTg-AD mice. The results of these studies add to the growing body of work examining the contribution of mitochondria to AD pathology. Many questions still remain to be examined, and future preclinical and clinical studies of mitochondria in AD and mitochondria-targeted treatments may yield new insight and overcome current barriers to slowing or halting AD progression. 


\section{Mitochondrial Changes Early in Development of Alzheimer's Disease}

Our study of embryonic primary cortical neurons of 3xTg-AD mice showed that mitochondrial movement and number were markedly deficient with modest structural abnormalities even at the very earliest stages of neuronal development. These observations were consistent with previous reports that exogenously applied A $\beta$ oligomers could impair mitochondrial movement and alter mitochondrial structure in primary neurons [18-20]. A strength of our study is that the endogenous production of $A \beta$ and potential synergistic interaction with hyperphosphorylated tau in 3xTg-AD neurons more closely mimics the conditions present in human $\mathrm{AD}$, particularly familial early-onset $\mathrm{AD}$ in which genetic mutations are present embryonically.

An implication of this study is that mitochondrial dysfunction during neuronal development could predispose individuals to be more susceptible to insults later in life that ultimately manifest as the neurodegeneration, synapse loss, brain atrophy, and cognitive decline that are hallmarks of AD. This could be an explanation of the phenomenon that some people do not develop the clinical symptoms of AD despite the presence of significant AD pathology [5, 6]. Conversely, one study of 430 elderly individuals found that 23 percent showed markers of brain injury typically associated with $\mathrm{AD}$, such as hippocampal atrophy and reduced cerebral glucose metabolism, without cerebral amyloid pathology [7]. The authors used the term "suspected nonAlzheimer pathophysiology" (sNAP) to describe this group, and it was notable that they showed many aspects of brain pathology including regional cerebral hypometabolism that were indistinguishable from patients classified with stage 2 and 3 preclinical AD.

These observations may be partially explained by the "reserve" hypotheses. "Brain reserve", "cognitive reserve", or "intellectual reserve" are terms interchangeably used to describe 
individuals that have a greater resilience to age-related cognitive decline, even in the face of $\mathrm{AD}$ pathology, due to more numerous neurons initially or increased capacity to recruit remaining neurons after they begin to degenerate [111, 126-129]. The proposed mechanisms behind increased cognitive reserve include increased education, increased socioeconomic status, and improved social networks. A complementary hypothesis of "metabolic reserve" which proposes that individuals with improved dietary, exercise, or other lifestyle factors are able to resist cognitive decline in the face of neuropathology due to improved metabolic functioning of remaining neural networks [112]. This hypothesis is supported by the association of MCI and $\mathrm{AD}$ with poor diet, obesity, type 2 diabetes, and lack of exercise.

While the reserve hypotheses described focus largely on environmental factors throughout life as being responsible for establishment of cognitive or metabolic reserves, we propose that early developmental factors may also play a role in establishing cognitive and metabolic reserves against neurodegenerative changes in AD. Early insults to mitochondrial number, structure, or function as seen in our study may negatively impact the proper development of neurons and synapses and lead to reduction in cognitive and/or metabolic reserves. While our study is most relevant in the case of familial AD due to the presence of mutant transgenes, other mitochondrial insults early in development could increase the risk for development of sporadic AD as well. Germline mutations in mitochondrial DNA cause premature brain aging and abnormalities in the cortex and hippocampus of adult mice [119].

Another possible early mitochondrial insult in the case of sporadic AD is expression of microRNA 34a. We have shown that microRNA 34a is elevated in the temporal cortex of human $\mathrm{AD}$ patients as well as the temporal cortex and hippocampus of 3xTg-AD mice [130]. Our preliminary studies showed that microRNA 34 a overexpression in primary cortical neurons of 
non-transgenic mice induced mitochondrial structural changes and deficits in mitochondrial movement (Cavendish et. al; unpublished observation). In primary hippocampal neurons, microRNA 34a overexpression causes reduction in ATP production, spare capacity, maximum respiration, and proton leak, and induces downregulation of proteins involved in oxidative phosphorylation [130]. Transgenic mice inducibly overexpressing microRNA 34a early in life develop cognitive deficits and AD histopathology in cortical and hippocampal regions (Sarkar et. al; publication in revision).

The implications of our research suggest that both familial early-onset and sporadic lateonset AD may stem from early impairment of mitochondrial function which diminishes cognitive/metabolic reserve and increase susceptibility to clinical dementia. This lends support to the exploration of early treatments to improve neuronal mitochondrial function for the prevention of $\mathrm{AD}$.

\section{Sigma-1 Receptor Agonists for Treatment of Alzheimer's Disease}

$\sigma 1 \mathrm{R}$ agonists are promising candidates for treatment of AD early in disease progression due to their known ability to increase mitochondrial ATP production among other neuroprotective effects [85]. In our study of mitochondrial deficits in embryonic 3xTg-AD neurons, we found that $\sigma 1 \mathrm{R}$ activation by $(+) \mathrm{SKF}-10,047$ can also increase neuronal mitochondrial movement and partially restore mitochondrial number deficits in 3xTg-AD neurons.

Although (+)SKF-10,047 is not approved for human use due to side effect profile, other $\sigma 1 \mathrm{R}$ agonists are available. ANAVEX2-73 is one such compound and is currently undergoing phase IIb/III clinical trials for treatment of MCI and early stage AD. Although the primary study 
is not expected to be completed until the end of 2020, the drug has passed its phase I and phase IIa trials showing high tolerability with minimal side effects. Other approved drugs with $\sigma 1 \mathrm{R}$ agonist activity include (+)Pentazocine, used for pain treatment, and dextromethorphan, used as a cough suppressant and treatment of pseudobulbar effect. Dextromethorphan has been shown to reduce agitation and aggression in $\mathrm{AD}$ patients, but has not been extensively studied as a diseasemodifying agent [131]. Memantine, an approved agent for AD treatment, also has partial $\sigma 1 \mathrm{R}$ agonist activity but also does not halt or slow disease progression.

Alzheimer's disease is a multifactorial and heterogeneous disease. $\sigma 1$ Rs may also alleviate $\mathrm{AD}$ through several other pathways. One factor of the pathogenesis of $\mathrm{AD}$ not examined in this dissertation is the contribution of inflammation and glial activation to neuronal damage and synapse loss [132]. It is known that several genes regulating glial clearance of misfolded proteins increase risk for sporadic $\mathrm{AD}$ [133]. A $\beta$ can bind to several microglial receptors and activate an innate immune response resulting in release of inflammatory cytokines and structural damage to neurons or synapses [134]. Increased cytokines also inhibit the microglial clearance of $A \beta$ from the brain [134]. Pretreatment with $\sigma 1 \mathrm{R}$ agonists has been shown to prevent microglial activation and attenuate cytokine release in response to multiple triggers of inflammation [135].

Oxidative stress is also associated with $\mathrm{AD}$ and brains of $\mathrm{AD}$ patients show increased lipid peroxidation [136]. Copper can bind to the n-terminus of $A \beta$, and the accumulation of copper in amyloid oligomers and plaques can trigger generation of reactive oxygen species [136]. Additionally, $A \beta$ accumulation causes increased zinc release and dysregulated zinc homeostasis in the brain, which also contributes to the increased production of reactive oxygen species [136]. $\sigma 1 \mathrm{R}$ agonists were shown to reduce amyloid-induced lipid peroxidation in the 
brains of mice [90] which is consistent with other observations of antioxidant properties of $\sigma 1 \mathrm{R}$ activation [85].

New compounds with $\sigma 1 \mathrm{R}$ agonist activity are continuously being developed which may provide promising results for early treatment of $\mathrm{AD}$ via modulation of mitochondrial activity among other diverse mechanisms of action [137-139]. More preclinical and clinical studies of $\sigma 1 \mathrm{R}$ agonists alone or in combination with other agents to combat $\mathrm{AD}$ are warranted.

\section{Preservation of Mitochondrial Structure and Number Late in Alzheimer's Disease}

Our study of aged 3xTg-AD mice showed surprisingly little change in mitochondrial structure across different brain areas and no change in mitochondrial number compared to nonTg controls. These findings contrasted with previous studies of human $\mathrm{AD}$ patients and $\mathrm{AD}$ mice which reported neuronal mitochondrial structural abnormalities [76-79] and reduction in neuronal mitochondrial number $[14,76]$ in cortical and hippocampal areas. We did find a modest reduction of mitochondrial volume in the CA1 area of the hippocampus in 3xTg-AD mice with increased sphericity, and it is known that the hippocampus is one of the earliest and most severely affected regions in AD. Subregion analysis showed that these changes were most heavily found in the stratum radiatum, where apical dendrites of hippocampal pyramidal neurons reside. This would be an expected result of reduced mitochondrial replenishment in distal dendrites due to reduced capacity for mitochondrial movement away from their site of biogenesis in the cell body, which is supported by in vitro data of primary neurons $[18,19]$.

M1 and S1 are typically spared from AD pathology, so an absence of mitochondrial abnormalities in those regions was not entirely unexpected. At 24 months, however, the $3 \times \mathrm{Tg}$ $\mathrm{AD}$ mouse shows considerable amyloid staining across the entire cortex and hippocampus 
(Figure 4.1). The lack of mitochondrial structural changes in the EC was unexpected given its high degree of involvement in AD pathology even at early stages of disease. The increased volume and reduced sphericity of AC mitochondria in the 3xTg-AD mouse could be consistent with the development of mitochondrial inclusions as are seen in the frontal cortical dendrites of AD patients [77].

Together, these findings indicate that mitochondria are largely intact in terms of structure and number at the latest stages of disease in 3xTg-AD mice. The discrepancies between our study and previous studies showing structural abnormalities could be due to the different imaging methods used. Using light microscopy to image large numbers of mitochondria unbiasedly in larger tissue volumes provided our study an advantage over previous studies which used electron microscopy to examine relatively small volumes of tissue and numbers of mitochondria. A disadvantage of our study was the inability to examine ultrastructural details of mitochondria which may have been present in 3xTg-AD mice.

Our study had several other limitations including the possibility that endogenous expression of mitochondrially-targeted CFP may have altered mitochondrial structure in both nonTg and 3xTg-AD mice. We were not able to test mitochondrial structure by light microscopy in non-CFP mice versus CFP-expressing mice due to lack of available antibodies to label mitochondria in fixed brain slices. In future studies, it would be possible to accomplish this with electron microscopy, but these studies were outside the scope of my dissertation work. Another limitation to this study was the small sample size available for study and the use of multiple ttests which can produce type-1 error. These limitations should be considered in the interpretation of study results. 
The lack of structural or number abnormalities found in our study suggest that the known metabolic changes associated with $\mathrm{AD}[3,67-70]$ are likely caused by dysfunction of the mitochondria that are present. This is promising for the treatment of AD with mitochondriallytargeted therapies even in mid to late stages of disease progression to protect remaining neurons. Oxaloacetate is the only current agent which specifically targets mitochondria undergoing clinical trials for $\mathrm{AD}$ treatment, and has completed phase I testing. The cerebral metabolic deficits which appear very early in $\mathrm{AD}$ progression combined with known mitochondrial functional deficits in $\mathrm{AD}$ highlight the need for more mitochondria-based treatment strategies. Mitochondrial enhancement, alone or in combination with other therapies, is a promising avenue for protecting neuronal function, preventing synapse loss, and slowing or halting the progression of $\mathrm{AD}$.

\section{Conclusions}

In over a century since its first description, $\mathrm{AD}$ has been a mysterious disease that has defied attempts to understand its pathology or produce therapies against it. The increasing elderly population, failure to develop disease-modifying treatments, and high burden of $\mathrm{AD}$ in terms of quality of life of patients and cost of care make $\mathrm{AD}$ one of the fastest growing healthcare problems of our society. Traditional approaches to treating AD based on amyloid pathology alone have all failed, and new strategies are needed. My dissertation work focused on understanding the mitochondrial aspects of $\mathrm{AD}$ in the early and late stages of disease. My work provided insights into the embryonic origins of $A D$ via mitochondrial deficits and suggested $\sigma 1 R$ activation as a potential therapeutic strategy for counteracting these deficits. I was able to show that mitochondrial structural and number alterations were not as severe as previously expected late in disease progression, which indicates that mitochondrial treatments may be able to be 
effective at salvaging neuronal function even in the later stages of disease. My preclinical research will hopefully inform future translational studies that may contribute to the development of new therapeutic strategies for treating $\mathrm{AD}$ and preventing disease progression in susceptible individuals. 

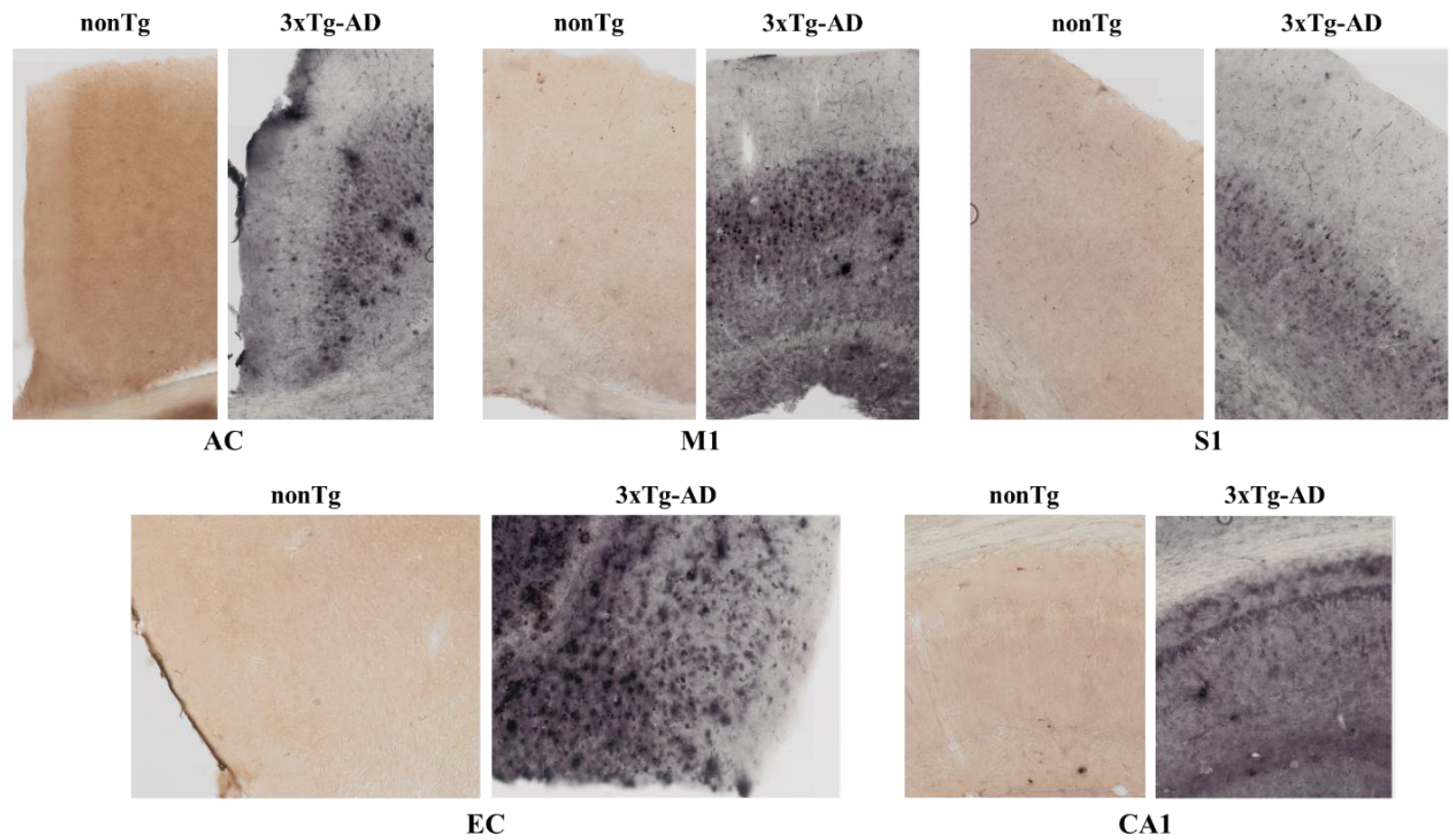

Figure 4.1. Amyloid staining in various brain areas of 3xTg-AD mice compared to nonTg controls at 24 months. Coronal sections stained with anti- $\beta$-amyloid $1-16$, clone $6 \mathrm{E} 10$ (Biolegend) shows considerable intracellular amyloid accumulation as well as extracellular plaques across cortical and hippocampal areas of 3xTg-AD mice. AC- Anterior Cingulate Cortex; M1- Primary Motor Cortex; S1- Primary Somatosensory Cortex; EC- Entorhinal Cortex; CA1- CA1 region of Hippocampus. 


\section{References:}

[1] Alzheimer's A (2018) 2018 Alzheimer's disease facts and figures. Alzheimers Dement 14, 367-429.

[2] Scheltens P, Blennow K, Breteler MM, de Strooper B, Frisoni GB, Salloway S, Van der Flier WM (2016) Alzheimer's disease. Lancet 388, 505-517.

[3] Mosconi L, Mistur R, Switalski R, Tsui WH, Glodzik L, Li Y, Pirraglia E, De Santi S, Reisberg B, Wisniewski T, de Leon MJ (2009) FDG-PET changes in brain glucose metabolism from normal cognition to pathologically verified Alzheimer's disease. Eur $J$ Nucl Med Mol Imaging 36, 811-822.

[4] Jia J, Sun B, Guo Z, Zhang J, Tian J, Tang H, Wang L (2011) Positron emission tomography with Pittsburgh compound B in diagnosis of early stage Alzheimer's disease. Cell Biochem Biophys 59, 57-62.

[5] SantaCruz KS, Sonnen JA, Pezhouh MK, Desrosiers MF, Nelson PT, Tyas SL (2011) Alzheimer disease pathology in subjects without dementia in 2 studies of aging: the Nun Study and the Adult Changes in Thought Study. J Neuropathol Exp Neurol 70, 832-840.

[6] Snowdon DA, Nun S (2003) Healthy aging and dementia: findings from the Nun Study. Ann Intern Med 139, 450-454.

[7] Knopman DS, Jack CR, Jr., Wiste HJ, Weigand SD, Vemuri P, Lowe VJ, Kantarci K, Gunter JL, Senjem ML, Mielke MM, Roberts RO, Boeve BF, Petersen RC (2013) Brain injury biomarkers are not dependent on beta-amyloid in normal elderly. Ann Neurol 73, 472-480.

[8] Lage JM (2006) 100 Years of Alzheimer's disease (1906-2006). J Alzheimers Dis 9, 1526.

[9] Alonso AD, Grundke-Iqbal I, Barra HS, Iqbal K (1997) Abnormal phosphorylation of tau and the mechanism of Alzheimer neurofibrillary degeneration: sequestration of microtubule-associated proteins 1 and 2 and the disassembly of microtubules by the abnormal tau. Proc Natl Acad Sci U S A 94, 298-303.

[10] Whitehouse PJ, Price DL, Clark AW, Coyle JT, DeLong MR (1981) Alzheimer disease: evidence for selective loss of cholinergic neurons in the nucleus basalis. Ann Neurol 10, 122-126.

[11] Perl DP (2010) Neuropathology of Alzheimer's disease. Mt Sinai J Med 77, 32-42.

[12] Thal DR, Ghebremedhin E, Orantes M, Wiestler OD (2003) Vascular pathology in Alzheimer disease: correlation of cerebral amyloid angiopathy and arteriosclerosis/lipohyalinosis with cognitive decline. J Neuropathol Exp Neurol 62, 1287-1301.

[13] Tuppo EE, Arias HR (2005) The role of inflammation in Alzheimer's disease. Int J Biochem Cell Biol 37, 289-305.

[14] Hirai K, Aliev G, Nunomura A, Fujioka H, Russell RL, Atwood CS, Johnson AB, Kress Y, Vinters HV, Tabaton M, Shimohama S, Cash AD, Siedlak SL, Harris PL, Jones PK, Petersen RB, Perry G, Smith MA (2001) Mitochondrial abnormalities in Alzheimer's disease. J Neurosci 21, 3017-3023.

[15] McKee AC, Cantu RC, Nowinski CJ, Hedley-Whyte ET, Gavett BE, Budson AE, Santini VE, Lee HS, Kubilus CA, Stern RA (2009) Chronic traumatic encephalopathy in athletes: progressive tauopathy after repetitive head injury. J Neuropathol Exp Neurol 68, 709735. 
[16] Hardy JA, Higgins GA (1992) Alzheimer's disease: the amyloid cascade hypothesis. Science 256, 184-185.

[17] Swerdlow RH, Burns JM, Khan SM (2014) The Alzheimer's disease mitochondrial cascade hypothesis: progress and perspectives. Biochim Biophys Acta 1842, 1219-1231.

[18] Sarkar S, Jun S, Simpkins JW (2015) Estrogen amelioration of Abeta-induced defects in mitochondria is mediated by mitochondrial signaling pathway involving ERbeta, AKAP and Drp1. Brain Res 1616, 101-111.

[19] Calkins MJ, Reddy PH (2011) Amyloid beta impairs mitochondrial anterograde transport and degenerates synapses in Alzheimer's disease neurons. Biochim Biophys Acta 1812, 507-513.

[20] Wang X, Perry G, Smith MA, Zhu X (2010) Amyloid-beta-derived diffusible ligands cause impaired axonal transport of mitochondria in neurons. Neurodegener Dis 7, 56-59.

[21] Hoerndli FJ, Pelech S, Papassotiropoulos A, Gotz J (2007) Abeta treatment and P301L tau expression in an Alzheimer's disease tissue culture model act synergistically to promote aberrant cell cycle re-entry. Eur J Neurosci 26, 60-72.

[22] LaFerla FM, Green KN (2012) Animal models of Alzheimer disease. Cold Spring Harb Perspect Med 2.

[23] Oddo S, Caccamo A, Shepherd JD, Murphy MP, Golde TE, Kayed R, Metherate R, Mattson MP, Akbari Y, LaFerla FM (2003) Triple-transgenic model of Alzheimer's disease with plaques and tangles: intracellular Abeta and synaptic dysfunction. Neuron 39, 409-421.

[24] Oddo S, Caccamo A, Kitazawa M, Tseng BP, LaFerla FM (2003) Amyloid deposition precedes tangle formation in a triple transgenic model of Alzheimer's disease. Neurobiol Aging 24, 1063-1070.

[25] Silver I, Erecinska M (1998) Oxygen and ion concentrations in normoxic and hypoxic brain cells. Adv Exp Med Biol 454, 7-16.

[26] Zhu XH, Qiao H, Du F, Xiong Q, Liu X, Zhang X, Ugurbil K, Chen W (2012) Quantitative imaging of energy expenditure in human brain. Neuroimage 60, 2107-2117.

[27] Erecinska M, Silver IA (1994) Ions and energy in mammalian brain. Prog Neurobiol 43, 37-71.

[28] Chang DT, Reynolds IJ (2006) Mitochondrial trafficking and morphology in healthy and injured neurons. Prog Neurobiol 80, 241-268.

[29] Rintoul GL, Reynolds IJ (2010) Mitochondrial trafficking and morphology in neuronal injury. Biochim Biophys Acta 1802, 143-150.

[30] Amiri M, Hollenbeck PJ (2008) Mitochondrial biogenesis in the axons of vertebrate peripheral neurons. Dev Neurobiol 68, 1348-1361.

[31] Chacinska A, Koehler CM, Milenkovic D, Lithgow T, Pfanner N (2009) Importing mitochondrial proteins: machineries and mechanisms. Cell 138, 628-644.

[32] Garesse R, Vallejo CG (2001) Animal mitochondrial biogenesis and function: a regulatory cross-talk between two genomes. Gene 263, 1-16.

[33] Chang DT, Honick AS, Reynolds IJ (2006) Mitochondrial trafficking to synapses in cultured primary cortical neurons. J Neurosci 26, 7035-7045.

[34] Popov V, Medvedev NI, Davies HA, Stewart MG (2005) Mitochondria form a filamentous reticular network in hippocampal dendrites but are present as discrete bodies in axons: a three-dimensional ultrastructural study. J Comp Neurol 492, 50-65. 
[35] Ohno N, Kidd GJ, Mahad D, Kiryu-Seo S, Avishai A, Komuro H, Trapp BD (2011) Myelination and axonal electrical activity modulate the distribution and motility of mitochondria at CNS nodes of Ranvier. J Neurosci 31, 7249-7258.

[36] Li Z, Okamoto K, Hayashi Y, Sheng M (2004) The importance of dendritic mitochondria in the morphogenesis and plasticity of spines and synapses. Cell 119, 873-887.

[37] Werth JL, Thayer SA (1994) Mitochondria buffer physiological calcium loads in cultured rat dorsal root ganglion neurons. J Neurosci 14, 348-356.

[38] Li P, Nijhawan D, Budihardjo I, Srinivasula SM, Ahmad M, Alnemri ES, Wang X (1997) Cytochrome $\mathrm{c}$ and dATP-dependent formation of Apaf-1/caspase-9 complex initiates an apoptotic protease cascade. Cell 91, 479-489.

[39] Pizzo P, Pozzan T (2007) Mitochondria-endoplasmic reticulum choreography: structure and signaling dynamics. Trends Cell Biol 17, 511-517.

[40] Gerencser AA, Nicholls DG (2008) Measurement of instantaneous velocity vectors of organelle transport: mitochondrial transport and bioenergetics in hippocampal neurons. Biophys J 95, 3079-3099.

[41] Ligon LA, Steward O (2000) Movement of mitochondria in the axons and dendrites of cultured hippocampal neurons. J Comp Neurol 427, 340-350.

[42] Misgeld T, Kerschensteiner M, Bareyre FM, Burgess RW, Lichtman JW (2007) Imaging axonal transport of mitochondria in vivo. Nat Methods 4, 559-561.

[43] Overly CC, Rieff HI, Hollenbeck PJ (1996) Organelle motility and metabolism in axons vs dendrites of cultured hippocampal neurons. J Cell Sci 109 ( Pt 5), 971-980.

[44] Sheng ZH (2014) Mitochondrial trafficking and anchoring in neurons: New insight and implications. J Cell Biol 204, 1087-1098.

[45] Sun T, Qiao H, Pan PY, Chen Y, Sheng ZH (2013) Motile axonal mitochondria contribute to the variability of presynaptic strength. Cell Rep 4, 413-419.

[46] Stowers RS, Megeath LJ, Gorska-Andrzejak J, Meinertzhagen IA, Schwarz TL (2002) Axonal transport of mitochondria to synapses depends on milton, a novel Drosophila protein. Neuron 36, 1063-1077.

[47] Fransson A, Ruusala A, Aspenstrom P (2003) Atypical Rho GTPases have roles in mitochondrial homeostasis and apoptosis. J Biol Chem 278, 6495-6502.

[48] Ligon LA, Steward O (2000) Role of microtubules and actin filaments in the movement of mitochondria in the axons and dendrites of cultured hippocampal neurons. J Comp Neurol 427, 351-361.

[49] Jung D, Filliol D, Miehe M, Rendon A (1993) Interaction of brain mitochondria with microtubules reconstituted from brain tubulin and MAP2 or TAU. Cell Motil Cytoskeleton 24, 245-255.

[50] Linden M, Nelson BD, Leterrier JF (1989) The specific binding of the microtubuleassociated protein 2 (MAP2) to the outer membrane of rat brain mitochondria. Biochem J 261, 167-173.

[51] Linden M, Nelson BD, Loncar D, Leterrier JF (1989) Studies on the interaction between mitochondria and the cytoskeleton. J Bioenerg Biomembr 21, 507-518.

[52] Price RL, Lasek RJ, Katz MJ (1991) Microtubules have special physical associations with smooth endoplasmic reticula and mitochondria in axons. Brain Res 540, 209-216.

[53] Kang JS, Tian JH, Pan PY, Zald P, Li C, Deng C, Sheng ZH (2008) Docking of axonal mitochondria by syntaphilin controls their mobility and affects short-term facilitation. Cell 132, 137-148. 
[54] Chen H, Detmer SA, Ewald AJ, Griffin EE, Fraser SE, Chan DC (2003) Mitofusins Mfn1 and Mfn2 coordinately regulate mitochondrial fusion and are essential for embryonic development. J Cell Biol 160, 189-200.

[55] Santel A, Fuller MT (2001) Control of mitochondrial morphology by a human mitofusin. J Cell Sci 114, 867-874.

[56] Alexander C, Votruba M, Pesch UE, Thiselton DL, Mayer S, Moore A, Rodriguez M, Kellner U, Leo-Kottler B, Auburger G, Bhattacharya SS, Wissinger B (2000) OPA1, encoding a dynamin-related GTPase, is mutated in autosomal dominant optic atrophy linked to chromosome 3q28. Nat Genet 26, 211-215.

[57] Shin HW, Shinotsuka C, Torii S, Murakami K, Nakayama K (1997) Identification and subcellular localization of a novel mammalian dynamin-related protein homologous to yeast Vps1p and Dnm1p. J Biochem 122, 525-530.

[58] Chappie JS, Mears JA, Fang S, Leonard M, Schmid SL, Milligan RA, Hinshaw JE, Dyda F (2011) A pseudoatomic model of the dynamin polymer identifies a hydrolysisdependent powerstroke. Cell 147, 209-222.

[59] Otera H, Wang C, Cleland MM, Setoguchi K, Yokota S, Youle RJ, Mihara K (2010) Mff is an essential factor for mitochondrial recruitment of Drp1 during mitochondrial fission in mammalian cells. J Cell Biol 191, 1141-1158.

[60] van der Bliek AM, Shen Q, Kawajiri S (2013) Mechanisms of mitochondrial fission and fusion. Cold Spring Harb Perspect Biol 5.

[61] Menzies RA, Gold PH (1971) The turnover of mitochondria in a variety of tissues of young adult and aged rats. J Biol Chem 246, 2425-2429.

[62] Kurihara Y, Kanki T, Aoki Y, Hirota Y, Saigusa T, Uchiumi T, Kang D (2012) Mitophagy plays an essential role in reducing mitochondrial production of reactive oxygen species and mutation of mitochondrial DNA by maintaining mitochondrial quantity and quality in yeast. $J$ Biol Chem 287, 3265-3272.

[63] Chu CT, Bayir H, Kagan VE (2014) LC3 binds externalized cardiolipin on injured mitochondria to signal mitophagy in neurons: implications for Parkinson disease. Autophagy 10, 376-378.

[64] Ashrafi G, Schlehe JS, LaVoie MJ, Schwarz TL (2014) Mitophagy of damaged mitochondria occurs locally in distal neuronal axons and requires PINK1 and Parkin. $J$ Cell Biol 206, 655-670.

[65] Atamna H, Frey WH, 2nd (2007) Mechanisms of mitochondrial dysfunction and energy deficiency in Alzheimer's disease. Mitochondrion 7, 297-310.

[66] Li Y, Rinne JO, Mosconi L, Pirraglia E, Rusinek H, DeSanti S, Kemppainen N, Nagren K, Kim BC, Tsui W, de Leon MJ (2008) Regional analysis of FDG and PIB-PET images in normal aging, mild cognitive impairment, and Alzheimer's disease. Eur J Nucl Med Mol Imaging 35, 2169-2181.

[67] Nicholson RM, Kusne Y, Nowak LA, LaFerla FM, Reiman EM, Valla J (2010) Regional cerebral glucose uptake in the 3xTG model of Alzheimer's disease highlights common regional vulnerability across AD mouse models. Brain Res 1347, 179-185.

[68] Bubber P, Haroutunian V, Fisch G, Blass JP, Gibson GE (2005) Mitochondrial abnormalities in Alzheimer brain: mechanistic implications. Ann Neurol 57, 695-703.

[69] Gibson GE, Haroutunian V, Zhang H, Park LC, Shi Q, Lesser M, Mohs RC, Sheu RK, Blass JP (2000) Mitochondrial damage in Alzheimer's disease varies with apolipoprotein E genotype. Ann Neurol 48, 297-303. 
[70] Yao J, Irwin RW, Zhao L, Nilsen J, Hamilton RT, Brinton RD (2009) Mitochondrial bioenergetic deficit precedes Alzheimer's pathology in female mouse model of Alzheimer's disease. Proc Natl Acad Sci U S A 106, 14670-14675.

[71] Gerschutz A, Heinsen H, Grunblatt E, Wagner AK, Bartl J, Meissner C, Fallgatter AJ, Al-Sarraj S, Troakes C, Ferrer I, Arzberger T, Deckert J, Riederer P, Fischer M, Tatschner T, Monoranu CM (2013) Neuron-specific mitochondrial DNA deletion levels in sporadic Alzheimer's disease. Curr Alzheimer Res 10, 1041-1046.

[72] Ghosh SS, Swerdlow RH, Miller SW, Sheeman B, Parker WD, Jr., Davis RE (1999) Use of cytoplasmic hybrid cell lines for elucidating the role of mitochondrial dysfunction in Alzheimer's disease and Parkinson's disease. Ann N Y Acad Sci 893, 176-191.

[73] Chen Y, Liang Z, Blanchard J, Dai CL, Sun S, Lee MH, Grundke-Iqbal I, Iqbal K, Liu F, Gong CX (2013) A non-transgenic mouse model (icv-STZ mouse) of Alzheimer's disease: similarities to and differences from the transgenic model (3xTg-AD mouse). $\mathrm{Mol}$ Neurobiol 47, 711-725.

[74] Correia SC, Santos RX, Santos MS, Casadesus G, Lamanna JC, Perry G, Smith MA, Moreira PI (2013) Mitochondrial abnormalities in a streptozotocin-induced rat model of sporadic Alzheimer's disease. Curr Alzheimer Res 10, 406-419.

[75] Stokin GB, Lillo C, Falzone TL, Brusch RG, Rockenstein E, Mount SL, Raman R, Davies P, Masliah E, Williams DS, Goldstein LS (2005) Axonopathy and transport deficits early in the pathogenesis of Alzheimer's disease. Science 307, 1282-1288.

[76] Baloyannis SJ (2006) Mitochondrial alterations in Alzheimer's disease. J Alzheimers Dis 9, 119-126.

[77] Saraiva AA, Borges MM, Madeira MD, Tavares MA, Paula-Barbosa MM (1985) Mitochondrial abnormalities in cortical dendrites from patients with Alzheimer's disease. J Submicrosc Cytol 17, 459-464.

[78] Zhang L, Trushin S, Christensen TA, Bachmeier BV, Gateno B, Schroeder A, Yao J, Itoh K, Sesaki H, Poon WW, Gylys KH, Patterson ER, Parisi JE, Diaz Brinton R, Salisbury JL, Trushina E (2016) Altered brain energetics induces mitochondrial fission arrest in Alzheimer's Disease. Sci Rep 6, 18725.

[79] Xie H, Guan J, Borrelli LA, Xu J, Serrano-Pozo A, Bacskai BJ (2013) Mitochondrial alterations near amyloid plaques in an Alzheimer's disease mouse model. J Neurosci 33, 17042-17051.

[80] Oppermann UC, Salim S, Tjernberg LO, Terenius L, Jornvall H (1999) Binding of amyloid beta-peptide to mitochondrial hydroxyacyl-CoA dehydrogenase (ERAB): regulation of an SDR enzyme activity with implications for apoptosis in Alzheimer's disease. FEBS Lett 451, 238-242.

[81] Anandatheerthavarada HK, Biswas G, Robin MA, Avadhani NG (2003) Mitochondrial targeting and a novel transmembrane arrest of Alzheimer's amyloid precursor protein impairs mitochondrial function in neuronal cells. J Cell Biol 161, 41-54.

[82] Qiao H, Koya RC, Nakagawa K, Tanaka H, Fujita H, Takimoto M, Kuzumaki N (2005) Inhibition of Alzheimer's amyloid-beta peptide-induced reduction of mitochondrial membrane potential and neurotoxicity by gelsolin. Neurobiol Aging 26, 849-855.

[83] Yan SD, Stern DM (2005) Mitochondrial dysfunction and Alzheimer's disease: role of amyloid-beta peptide alcohol dehydrogenase (ABAD). Int J Exp Pathol 86, 161-171.

[84] Rui Y, Tiwari P, Xie Z, Zheng JQ (2006) Acute impairment of mitochondrial trafficking by beta-amyloid peptides in hippocampal neurons. J Neurosci 26, 10480-10487. 
[85] Nguyen L, Lucke-Wold BP, Mookerjee SA, Cavendish JZ, Robson MJ, Scandinaro AL, Matsumoto RR (2015) Role of sigma-1 receptors in neurodegenerative diseases. $J$ Pharmacol Sci 127, 17-29.

[86] Hayashi T, Rizzuto R, Hajnoczky G, Su TP (2009) MAM: more than just a housekeeper. Trends Cell Biol 19, 81-88.

[87] Hayashi T, Su TP (2007) Sigma-1 receptor chaperones at the ER-mitochondrion interface regulate $\mathrm{Ca}(2+)$ signaling and cell survival. Cell 131, 596-610.

[88] Maurice T, Su TP, Privat A (1998) Sigma1 (sigma 1) receptor agonists and neurosteroids attenuate B25-35-amyloid peptide-induced amnesia in mice through a common mechanism. Neuroscience 83, 413-428.

[89] Meunier J, Ieni J, Maurice T (2006) The anti-amnesic and neuroprotective effects of donepezil against amyloid beta25-35 peptide-induced toxicity in mice involve an interaction with the sigma1 receptor. Br J Pharmacol 149, 998-1012.

[90] Villard V, Espallergues J, Keller E, Alkam T, Nitta A, Yamada K, Nabeshima T, Vamvakides A, Maurice T (2009) Antiamnesic and neuroprotective effects of the aminotetrahydrofuran derivative ANAVEX1-41 against amyloid beta(25-35)-induced toxicity in mice. Neuropsychopharmacology 34, 1552-1566.

[91] Mori T, Hayashi T, Hayashi E, Su TP (2013) Sigma-1 receptor chaperone at the ERmitochondrion interface mediates the mitochondrion-ER-nucleus signaling for cellular survival. PLoS One 8, e76941.

[92] Behensky AA, Yasny IE, Shuster AM, Seredenin SB, Petrov AV, Cuevas J (2013) Afobazole activation of sigma-1 receptors modulates neuronal responses to amyloidbeta25-35. J Pharmacol Exp Ther 347, 468-477.

[93] Behensky AA, Yasny IE, Shuster AM, Seredenin SB, Petrov AV, Cuevas J (2013) Stimulation of sigma receptors with afobazole blocks activation of microglia and reduces toxicity caused by amyloid-beta25-35. J Pharmacol Exp Ther 347, 458-467.

[94] Lahmy V, Meunier J, Malmstrom S, Naert G, Givalois L, Kim SH, Villard V, Vamvakides A, Maurice T (2013) Blockade of Tau hyperphosphorylation and Abeta(1)()(4)(2) generation by the aminotetrahydrofuran derivative ANAVEX2-73, a mixed muscarinic and sigma(1) receptor agonist, in a nontransgenic mouse model of Alzheimer's disease. Neuropsychopharmacology 38, 1706-1723.

[95] Hardy J, Selkoe DJ (2002) The amyloid hypothesis of Alzheimer's disease: progress and problems on the road to therapeutics. Science 297, 353-356.

[96] Maccioni RB, Farias G, Morales I, Navarrete L (2010) The revitalized tau hypothesis on Alzheimer's disease. Arch Med Res 41, 226-231.

[97] Iqbal K, Grundke-Iqbal I (2010) Alzheimer's disease, a multifactorial disorder seeking multitherapies. Alzheimers Dement 6, 420-424.

[98] Cummings J, Lee G, Ritter A, Zhong K (2018) Alzheimer's disease drug development pipeline: 2018. Alzheimers Dement (N Y) 4, 195-214.

[99] Gong CX, Liu F, Iqbal K (2018) Multifactorial Hypothesis and Multi-Targets for Alzheimer's Disease. J Alzheimers Dis 64, S107-S117.

[100] Andrade-Moraes CH, Oliveira-Pinto AV, Castro-Fonseca E, da Silva CG, Guimaraes DM, Szczupak D, Parente-Bruno DR, Carvalho LR, Polichiso L, Gomes BV, Oliveira LM, Rodriguez RD, Leite RE, Ferretti-Rebustini RE, Jacob-Filho W, Pasqualucci CA, Grinberg LT, Lent R (2013) Cell number changes in Alzheimer's disease relate to dementia, not to plaques and tangles. Brain 136, 3738-3752. 
[101] Giannakopoulos P, Kovari E, Gold G, von Gunten A, Hof PR, Bouras C (2009) Pathological substrates of cognitive decline in Alzheimer's disease. Front Neurol Neurosci 24, 20-29.

[102] Santos RX, Correia SC, Wang X, Perry G, Smith MA, Moreira PI, Zhu X (2010) Alzheimer's disease: diverse aspects of mitochondrial malfunctioning. Int J Clin Exp Pathol 3, 570-581.

[103] Wang X, Su B, Siedlak SL, Moreira PI, Fujioka H, Wang Y, Casadesus G, Zhu X (2008) Amyloid-beta overproduction causes abnormal mitochondrial dynamics via differential modulation of mitochondrial fission/fusion proteins. Proc Natl Acad Sci U S A 105, 19318-19323.

[104] Phillips NR, Simpkins JW, Roby RK (2014) Mitochondrial DNA deletions in Alzheimer's brains: a review. Alzheimers Dement 10, 393-400.

[105] Vale C, Alonso E, Rubiolo JA, Vieytes MR, LaFerla FM, Gimenez-Llort L, Botana LM (2010) Profile for amyloid-beta and tau expression in primary cortical cultures from 3xTg-AD mice. Cell Mol Neurobiol 30, 577-590.

[106] Lewis A, Tsai SY, Su TP (2016) Detection of Isolated Mitochondria-Associated ER Membranes Using the Sigma-1 Receptor. Methods Mol Biol 1376, 133-140.

[107] Sheng ZH, Cai Q (2012) Mitochondrial transport in neurons: impact on synaptic homeostasis and neurodegeneration. Nat Rev Neurosci 13, 77-93.

[108] Weng TY, Tsai SA, Su TP (2017) Roles of sigma-1 receptors on mitochondrial functions relevant to neurodegenerative diseases. J Biomed Sci 24, 74.

[109] DeCoster MA, Klette KL, Knight ES, Tortella FC (1995) Sigma receptor-mediated neuroprotection against glutamate toxicity in primary rat neuronal cultures. Brain Res 671, 45-53.

[110] McClatchey PM, Keller AC, Bouchard R, Knaub LA, Reusch JE (2016) Fully automated software for quantitative measurements of mitochondrial morphology. Mitochondrion 26, 58-71.

[111] Mortimer JA, Borenstein AR, Gosche KM, Snowdon DA (2005) Very early detection of Alzheimer neuropathology and the role of brain reserve in modifying its clinical expression. J Geriatr Psychiatry Neurol 18, 218-223.

[112] Stranahan AM, Mattson MP (2012) Metabolic reserve as a determinant of cognitive aging. J Alzheimers Dis 30 Suppl 2, S5-13.

[113] Natsvlishvili N, Goguadze N, Zhuravliova E, Mikeladze D (2015) Sigma-1 receptor directly interacts with Rac1-GTPase in the brain mitochondria. BMC Biochem 16, 11.

[114] Jornayvaz FR, Shulman GI (2010) Regulation of mitochondrial biogenesis. Essays Biochem 47, 69-84.

[115] Vollrath JT, Sechi A, Dreser A, Katona I, Wiemuth D, Vervoorts J, Dohmen M, Chandrasekar A, Prause J, Brauers E, Jesse CM, Weis J, Goswami A (2014) Loss of function of the ALS protein SigR1 leads to ER pathology associated with defective autophagy and lipid raft disturbances. Cell Death Dis 5, e1290.

[116] Puleston D (2015) Detection of Mitochondrial Mass, Damage, and Reactive Oxygen Species by Flow Cytometry. Cold Spring Harb Protoc 2015, pdb prot086298.

[117] Soejima N, Ohyagi Y, Nakamura N, Himeno E, Iinuma KM, Sakae N, Yamasaki R, Tabira T, Murakami K, Irie K, Kinoshita N, LaFerla FM, Kiyohara Y, Iwaki T, Kira J (2013) Intracellular accumulation of toxic turn amyloid-beta is associated with endoplasmic reticulum stress in Alzheimer's disease. Curr Alzheimer Res 10, 11-20. 
[118] Omi T, Tanimukai H, Kanayama D, Sakagami Y, Tagami S, Okochi M, Morihara T, Sato M, Yanagida K, Kitasyoji A, Hara H, Imaizumi K, Maurice T, Chevallier N, Marchal S, Takeda M, Kudo T (2014) Fluvoxamine alleviates ER stress via induction of Sigma-1 receptor. Cell Death Dis 5, e1332.

[119] Ross JM, Stewart JB, Hagstrom E, Brene S, Mourier A, Coppotelli G, Freyer C, Lagouge M, Hoffer BJ, Olson L, Larsson NG (2013) Germline mitochondrial DNA mutations aggravate ageing and can impair brain development. Nature 501, 412-415.

[120] Lewis TL, Jr., Kwon SK, Lee A, Shaw R, Polleux F (2018) MFF-dependent mitochondrial fission regulates presynaptic release and axon branching by limiting axonal mitochondria size. Nat Commun 9, 5008.

[121] Chang DT, Reynolds IJ (2006) Differences in mitochondrial movement and morphology in young and mature primary cortical neurons in culture. Neuroscience 141, 727-736.

[122] Wirths O, Bayer TA (2010) Neuron loss in transgenic mouse models of Alzheimer's disease. Int J Alzheimers Dis 2010.

[123] Quintana DD, Garcia JA, Sarkar SN, Jun S, Engler-Chiurazzi EB, Russell AE, Cavendish JZ, Simpkins JW (2018) Hypoxia-reoxygenation of primary astrocytes results in a redistribution of mitochondrial size and mitophagy. Mitochondrion.

[124] Kislin M, Sword J, Fomitcheva IV, Croom D, Pryazhnikov E, Lihavainen E, Toptunov D, Rauvala H, Ribeiro AS, Khiroug L, Kirov SA (2017) Reversible Disruption of Neuronal Mitochondria by Ischemic and Traumatic Injury Revealed by Quantitative Two-Photon Imaging in the Neocortex of Anesthetized Mice. J Neurosci 37, 333-348.

[125] Moreira PI, Carvalho C, Zhu X, Smith MA, Perry G (2010) Mitochondrial dysfunction is a trigger of Alzheimer's disease pathophysiology. Biochim Biophys Acta 1802, 2-10.

[126] Alexander GE, Furey ML, Grady CL, Pietrini P, Brady DR, Mentis MJ, Schapiro MB (1997) Association of premorbid intellectual function with cerebral metabolism in Alzheimer's disease: implications for the cognitive reserve hypothesis. Am J Psychiatry 154, 165-172.

[127] Katzman R (1993) Education and the prevalence of dementia and Alzheimer's disease. Neurology 43, 13-20.

[128] Katzman R, Terry R, DeTeresa R, Brown T, Davies P, Fuld P, Renbing X, Peck A (1988) Clinical, pathological, and neurochemical changes in dementia: a subgroup with preserved mental status and numerous neocortical plaques. Ann Neurol 23, 138-144.

[129] Stern Y, Gurland B, Tatemichi TK, Tang MX, Wilder D, Mayeux R (1994) Influence of education and occupation on the incidence of Alzheimer's disease. JAMA 271, 10041010.

[130] Sarkar S, Jun S, Rellick S, Quintana DD, Cavendish JZ, Simpkins JW (2016) Expression of microRNA-34a in Alzheimer's disease brain targets genes linked to synaptic plasticity, energy metabolism, and resting state network activity. Brain Res 1646, 139-151.

[131] Cummings JL, Lyketsos CG, Peskind ER, Porsteinsson AP, Mintzer JE, Scharre DW, De La Gandara JE, Agronin M, Davis CS, Nguyen U, Shin P, Tariot PN, Siffert J (2015) Effect of Dextromethorphan-Quinidine on Agitation in Patients With Alzheimer Disease Dementia: A Randomized Clinical Trial. JAMA 314, 1242-1254.

[132] Griffin WS, Sheng JG, Royston MC, Gentleman SM, McKenzie JE, Graham DI, Roberts GW, Mrak RE (1998) Glial-neuronal interactions in Alzheimer's disease: the potential role of a 'cytokine cycle' in disease progression. Brain Pathol 8, 65-72. 
[133] Frost GR, Li YM (2017) The role of astrocytes in amyloid production and Alzheimer's disease. Open Biol 7.

[134] Sarlus H, Heneka MT (2017) Microglia in Alzheimer's disease. J Clin Invest 127, 32403249.

[135] Nguyen L, Lucke-Wold BP, Mookerjee S, Kaushal N, Matsumoto RR (2017) Sigma-1 Receptors and Neurodegenerative Diseases: Towards a Hypothesis of Sigma-1 Receptors as Amplifiers of Neurodegeneration and Neuroprotection. Adv Exp Med Biol 964, 133152.

[136] Christen Y (2000) Oxidative stress and Alzheimer disease. Am J Clin Nutr 71, 621S$629 \mathrm{~S}$.

[137] Estrada M, Perez C, Soriano E, Laurini E, Romano M, Pricl S, Morales-Garcia JA, PerezCastillo A, Rodriguez-Franco MI (2016) New neurogenic lipoic-based hybrids as innovative Alzheimer's drugs with sigma-1 agonism and beta-secretase inhibition. Future Med Chem 8, 1191-1207.

[138] Fisher A, Bezprozvanny I, Wu L, Ryskamp DA, Bar-Ner N, Natan N, Brandeis R, Elkon H, Nahum V, Gershonov E, LaFerla FM, Medeiros R (2016) AF710B, a Novel M1/sigma1 Agonist with Therapeutic Efficacy in Animal Models of Alzheimer's Disease. Neurodegener Dis 16, 95-110.

[139] Hall H, Iulita MF, Gubert P, Flores Aguilar L, Ducatenzeiler A, Fisher A, Cuello AC (2018) AF710B, an M1/sigma-1 receptor agonist with long-lasting disease-modifying properties in a transgenic rat model of Alzheimer's disease. Alzheimers Dement 14, 811823. 